\title{
Selective anchoring groups for molecular electronic junctions with ITO electrodes
}

Inco J. Planje, ${ }^{\text {a†* }}$ Ross J. Davidson, ${ }^{b}$ Andrea Vezzoli, ${ }^{a}$ Abdalghani Daaoub, ${ }^{c}$ Sara Sangtarash, ${ }^{\text {cd }}$ Hatef Sadeghi, ${ }^{c}$ Santiago Martín, ${ }^{\text {ef }}$ Pilar Cea,${ }^{\text {ef }}$ Colin J. Lambert, ${ }^{d}$ Andrew Beeby, ${ }^{\mathrm{b}}$ Simon J. Higgins, ${ }^{\mathrm{a}}$ and Richard J. Nichols ${ }^{\mathrm{a} *}$

${ }^{a}$ Department of Chemistry, University of Liverpool, Crown St, Liverpool, L69 7ZD, UK

${ }^{b}$ Department of Chemistry, Durham University, South Rd, Durham, DH1 3LE, UK

${ }^{c}$ School of Engineering, University of Warwick, Coventry CV4 7AL, UK

${ }^{d}$ Department of Physics, Lancaster University, Lancaster LA1 4YW, UK

e Departamento de Química Física, Facultad de Ciencias, Universidad de Zaragoza, Spain

${ }^{f}$ Instituto de Nanociencia y Materiales de Aragón (INMA), Universidad de Zaragoza-CSIC, 50009 Zaragoza, Spain

† Present address: Department of Chemistry, King's College London, 7 Trinity Street, London SE1 1DB, UK

*Corresponding authors: inco.planje@kcl.ac.uk, nichols@liverpool.ac.uk 


\section{Contents}

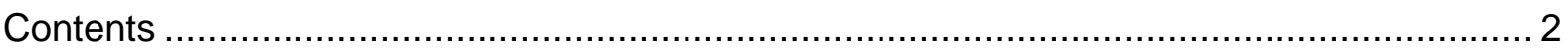

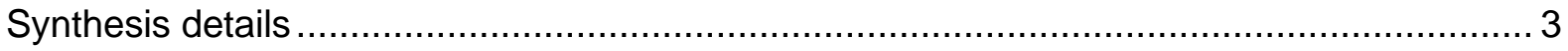

S1. Synthesis and characterisation of target compounds ...................................... 3

S2. NMR spectra of reported compounds .................................................................. 11

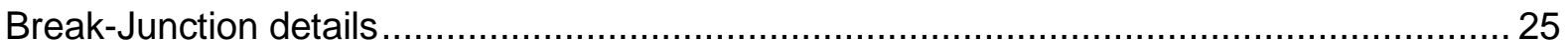

Absorption spectroscopy of wires 6 -carboxylate, 7 -acrylate, and 8 -squarate ....................... 27

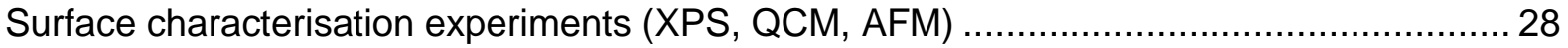

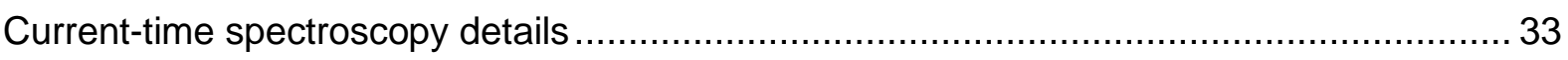

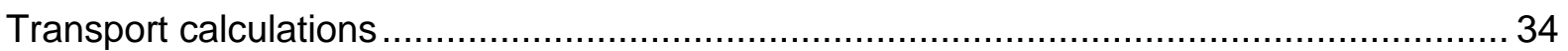

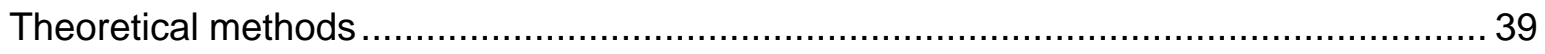

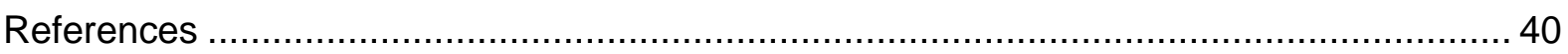




\section{Synthesis details}

\section{S1. Synthesis and characterisation of target compounds}

General details: NMR spectra were recorded in deuterated solvent solutions on a Varian VNMRS-600 spectrometer and referenced against solvent resonances $\left({ }^{1} \mathrm{H},{ }^{13} \mathrm{C}\right)$. ASAP data were recorded using a Xevo QTOF (Waters) high resolution, accurate mass tandem mass spectrometer equipped with Atmospheric Pressure Gas Chromatography (APGC) and Atmospheric Solids Analysis Probe (ASAP). Microanalyses were performed by the Elemental Microanalysis service, Durham University, UK. All chemicals were sourced from standard chemical suppliers, except 4-((4-(acetylthio)phenyl)ethynyl)benzoic acid (6carboxylate) ${ }^{1}, \quad$ 5-bromo-3,3-dimethyl-2,3-dihydrobenzothiophene, ${ }^{2}$

ethynylphenyl)(methyl)sulfane ${ }^{3}$-hydroxy-3-(4-iodophenyl)pent-3-en-2-one, ${ }^{4}$ 3-hydroxy-1(4-iodophenyl)but-2-en-1-one, ${ }^{5}$ and S-(4-(pyridin-4-ylethynyl)phenyl) ethanethioate ${ }^{6}$ which were synthesised according to published methods.

methyl-4-((3,3-dimethyl-2,3-dihydrobenzothiophen-5-yl)ethynyl)benzoate

(a). $\mathrm{Pd}\left(\mathrm{PPh}_{3}\right)_{4}(300 \mathrm{mg}, 0.26 \mathrm{mmol})$ and $\mathrm{CuI}(49 \mathrm{mg}, 0.26 \mathrm{mmol})$ were added to a freeze-pumpthaw degassed solution containing 5-ethynyl-3,3-dimethyl-2,3-dihydrobenzothiophene (500 $\mathrm{mg}, 2.65 \mathrm{mmol})$, methyl 4-iodobenzoate (696 mg, $2.65 \mathrm{mmol}), \mathrm{Et}_{3} \mathrm{~N}(5 \mathrm{~mL})$ and THF (20 $\mathrm{mL}$ ). The solution was stirred for $16 \mathrm{~h}$ before the solvent was removed. Purification was achieved by column chromatography eluted by a solvent gradient from DCM:hexane (1:1) to neat DCM forming a white solid. Yield: $0.39 \mathrm{~g} \mathrm{(46 \% ).}{ }^{1} \mathbf{H}-\mathbf{N M R}\left(\mathrm{CDCl}_{3}, 600 \mathrm{MHz}\right) \delta 8.00$ $\left(\mathrm{d},{ }^{3} J_{\mathrm{HH}}=8.17 \mathrm{~Hz}, 2 \mathrm{H}, \mathrm{H}_{\mathrm{e}}\right), 7.56\left(\mathrm{~d},{ }^{3} J_{\mathrm{HH}}=8.13 \mathrm{~Hz}, 2 \mathrm{H}, \mathrm{H}_{\mathrm{d}}\right), 7.30\left(\mathrm{dd},{ }^{3} J_{\mathrm{HH}}=7.9,{ }^{4} J_{\mathrm{HH}}=1.6\right.$ $\left.\mathrm{Hz}, 1 \mathrm{H}, \mathrm{H}_{\mathrm{b}}\right), 7.20\left(\mathrm{~d},{ }^{4} J_{\mathrm{HH}}=1.6 \mathrm{~Hz}, 1 \mathrm{H}, \mathrm{H}_{\mathrm{a}}\right), 7.16\left(\mathrm{~d},{ }^{3} J_{\mathrm{HH}}=8.0 \mathrm{~Hz}, 1 \mathrm{H}, \mathrm{H}_{\mathrm{c}}\right), 3.92\left(\mathrm{~s}, 3 \mathrm{H}, \mathrm{H}_{\mathrm{f}}\right)$, $3.20\left(\mathrm{~s}, 2 \mathrm{H}, \mathrm{H}_{\mathrm{h}}\right), 1.39$ (s, 6H, Hg) ppm. ${ }^{13} \mathbf{C}\left\{{ }^{1} \mathbf{H}\right\}-\mathbf{N M R}\left(\mathrm{CDCl}_{3}, 126 \mathrm{MHz}\right) \delta: 166.5,148.3$, $142.3,131.3,130.9,129.4,129.2,128.1,125.9,122.3,118.4,92.8,88.1,52.1,47.2,27.3$ ppm. MS(ASAP): $m / z 323.097[\mathrm{M}+\mathrm{H}]^{+}$. Anal. Calc. for $\mathrm{C}_{20} \mathrm{H}_{18} \mathrm{O}_{2} \mathrm{~S}: \mathrm{C}, 74.50 ; \mathrm{H}, 5.63 \%$. Found: C,74.11; H, 5.69\%.

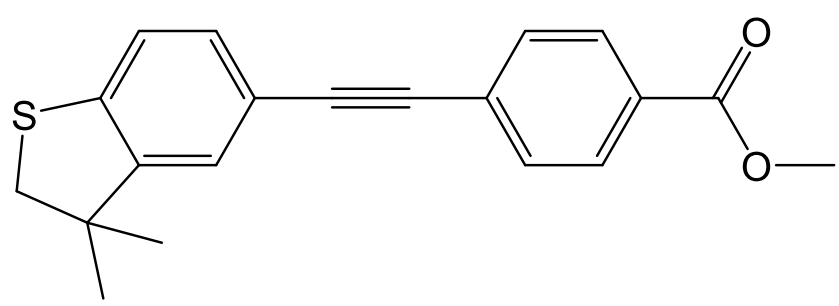


4-((3,3-dimethyl-2,3-dihydrobenzo[b]thiophen-5-yl)ethynyl)benzoic acid (1). To a solution containing a (500 mg, $1.55 \mathrm{mmol})$ in THF $(30 \mathrm{~mL}), \mathrm{KOH}(173 \mathrm{mg}, 3.1 \mathrm{mmol})$ in $\mathrm{H}_{2} \mathrm{O}(10 \mathrm{ml})$ was added. The solution was stirred at room temperature for $8 \mathrm{~h}$ before the solution was acidified with $\mathrm{HCl}$ followed by extraction with $\mathrm{DCM}$, dried over $\mathrm{MgSO}_{4}$ before removing the solvent. Yield: $0.38 \mathrm{~g}(81 \%) .{ }^{1} \mathrm{H}-\mathrm{NMR}\left(\mathrm{DMSO}-\mathrm{d}_{6}, 600 \mathrm{MHz}\right) \delta 13.14(\mathrm{~s}, 1 \mathrm{H}$, $\left.\mathrm{H}_{\mathrm{f}}\right), 7.97\left(\mathrm{~d},{ }^{3} \mathrm{~J}_{\mathrm{HH}}=8.5 \mathrm{~Hz}, 2 \mathrm{H}, \mathrm{H}_{\mathrm{e}}\right), 7.65\left(\mathrm{~d},{ }^{3} J_{\mathrm{HH}}=8.5 \mathrm{~Hz}, 2 \mathrm{H}, \mathrm{H}_{\mathrm{d}}\right), 7.37-7.34(\mathrm{~m}, 2 \mathrm{H}$, $\left.\mathrm{H}_{\mathrm{a}}+\mathrm{H}_{\mathrm{b}}\right), 7.29\left(\mathrm{~d},{ }^{3} J_{\mathrm{HH}}=7.8 \mathrm{~Hz}, 1 \mathrm{H}, \mathrm{H}_{\mathrm{c}}\right), 3.25\left(\mathrm{~s}, 2 \mathrm{H}, \mathrm{H}_{\mathrm{h}}\right), 1.35\left(\mathrm{~s}, 6 \mathrm{H}, \mathrm{H}_{\mathrm{g}}\right) \mathrm{ppm} \cdot{ }^{13} \mathbf{C}\left\{{ }^{1} \mathbf{H}\right\}-$ NMR (DMSO-d $\left.\mathrm{d}_{6}, 126 \mathrm{MHz}\right) \delta: 149.2,142.6,131.8,131.3,130.0,127.3,126.4,122.9$, 118.0, 93.0, 88.6, 47.3, 46.8, 27.2 ppm. MS(ASAP): $\mathrm{m} / z$ 309.091 $[\mathrm{M}+\mathrm{H}]^{+}$. Anal. Calc. for $\mathrm{C}_{19} \mathrm{H}_{16} \mathrm{O}_{2} \mathrm{~S} \cdot 1 / 2 \mathrm{H}_{2} \mathrm{O}: \mathrm{C}, 71.90 ; \mathrm{H}, 5.40 \%$. Found: $\mathrm{C}, 71.63 ; \mathrm{H}, 5.13 \%$

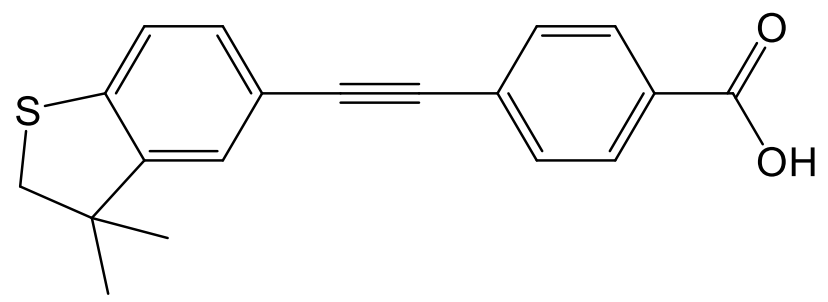

\section{3-(4-((3,3-dimethyl-2,3-dihydrobenzo[b]thiophen-5-yl)ethynyl)phenyl)-4-}

hydroxypent-3-en-2-one (2). $\mathrm{Pd}\left(\mathrm{PPh}_{3}\right)_{4}(381 \mathrm{mg}, 0.33 \mathrm{mmol})$ and $\mathrm{CuI}(62 \mathrm{mg}, 0.33 \mathrm{mmol})$ were added to a freeze-pump-thaw degassed solution containing (4ethynylphenyl)(methyl)sulfane (500 mg, $3.37 \mathrm{mmol}$ ), 4-hydroxy-3-(4-iodophenyl)pent-3-en2-one (1.01 g, $3.37 \mathrm{mmol}), \mathrm{Et}_{3} \mathrm{~N}(5 \mathrm{~mL})$, and THF (20 mL). The solution was stirred for $16 \mathrm{~h}$ before the solvent was removed. Purification was achieved by column chromatography eluted by a solvent gradient from DCM:hexane (1:1) to neat DCM forming a white solid. Yield: $0.38 \mathrm{~g}(35 \%) .{ }^{1} \mathbf{H}-\mathbf{N M R}\left(\mathrm{CDCl}_{3}, 600 \mathrm{MHz}\right) \delta 7.54\left(\mathrm{~d},{ }^{3} J_{\mathrm{HH}}=7.9 \mathrm{~Hz}, 2 \mathrm{H}, \mathrm{H}_{\mathrm{d}}\right), 7.44\left(\mathrm{~d},{ }^{3} J_{\mathrm{HH}}=\right.$ $\left.8.3 \mathrm{~Hz}, 2 \mathrm{H}, \mathrm{H}_{\mathrm{a}}\right), 7.21\left(\mathrm{~d},{ }^{3} J_{\mathrm{HH}}=8.3 \mathrm{~Hz}, 2 \mathrm{H}, \mathrm{H}_{\mathrm{b}}\right), 7.16\left(\mathrm{~d},{ }^{3} J_{\mathrm{HH}}=8.3 \mathrm{~Hz}, 2 \mathrm{H}, \mathrm{H}_{\mathrm{c}}\right), 2.50(\mathrm{~s}, 3 \mathrm{H}$, $\left.\mathrm{H}_{\mathrm{g}}\right), 1.89\left(\mathrm{~s}, 6 \mathrm{H}, \mathrm{H}_{\mathrm{h}}\right) \mathrm{ppm} .{ }^{13} \mathbf{C}\left\{{ }^{1} \mathbf{H}\right\}-\mathbf{N M R}\left(\mathrm{CDCl}_{3}, 126 \mathrm{MHz}\right) \delta: 190.7,139.5,136.8,131.9$, $131.8,131.1,125.8,122.5,119.2,114.7,89.8,88.9,24.1,15.3$ ppm. MS(ASAP): $\mathrm{m} / \mathrm{z}$ $323.090[\mathrm{M}+\mathrm{H}]^{+}$. Anal. Calc. for $\mathrm{C}_{20} \mathrm{H}_{18} \mathrm{O}_{2} \mathrm{~S} \cdot 1 / 4 \mathrm{H}_{2} \mathrm{O}$ : C, 73.48; H, 5.70\%. Found: C, 73.36; $\mathrm{H}, 5.63 \%$.

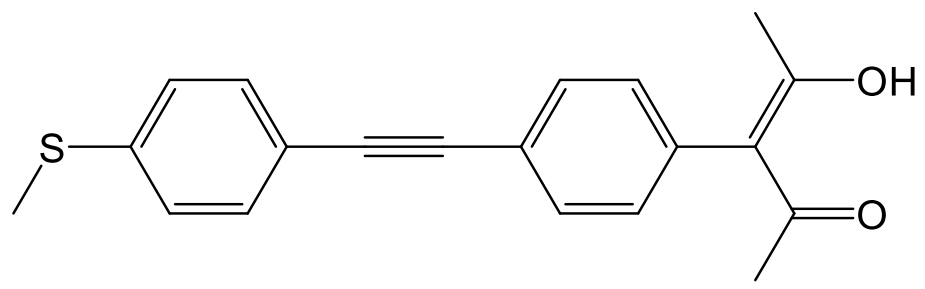


4-((3,3-dimethyl-2,3- dihydrobenzothiophen -5-yl)ethynyl)pyridine (b). $\mathrm{Pd}\left(\mathrm{PPh}_{3}\right)_{4}$ (300 mg, $0.26 \mathrm{mmol}$ ) and $\mathrm{CuI}(49 \mathrm{mg}, 0.26 \mathrm{mmol})$ were added to a freeze-pump-thaw degassed solution containing 5-ethynyl-3,3-dimethyl-2,3-dihydrobenzothiophene $(500 \mathrm{mg}$, $2.65 \mathrm{mmol})$, 4-iodopyridine (545 mg, $2.65 \mathrm{mmol}), \mathrm{Et}_{3} \mathrm{~N}(5 \mathrm{~mL})$ and THF (20 mL). The solution was stirred for $16 \mathrm{~h}$ before the solvent was removed. Purification was achieved by column chromatography eluted by a solvent gradient with neat DCM to neat diethylether, producing a colourless oil. Yield: $0.60 \mathrm{~g}(86 \%) .{ }^{1} \mathbf{H}-\mathbf{N M R}\left(\mathrm{CDCl}_{3}, 600 \mathrm{MHz}\right) \delta 8.61$ (br, $2 \mathrm{H}$, $\left.\mathrm{H}_{\mathrm{e}}\right), 7.36\left(\mathrm{br}, 2 \mathrm{H}, \mathrm{H}_{\mathrm{d}}\right), 7.30\left(\mathrm{dd},{ }^{3} J_{\mathrm{HH}}=7.96 \mathrm{~Hz},{ }^{4} J_{\mathrm{HH}}=1.62 \mathrm{~Hz}, 1 \mathrm{H}, \mathrm{H}_{\mathrm{b}}\right), 7.19\left(\mathrm{~d},{ }^{4} J_{\mathrm{HH}}=1.59\right.$ $\left.\mathrm{Hz}, 1 \mathrm{H}, \mathrm{H}_{\mathrm{a}}\right), 7.16\left(\mathrm{~d},{ }^{3} \mathrm{~J}_{\mathrm{HH}}=8.0 \mathrm{~Hz}, 2 \mathrm{H}, \mathrm{H}_{\mathrm{c}}\right), 3.19\left(\mathrm{~s}, 2 \mathrm{H}, \mathrm{H}_{\mathrm{g}}\right), 1.38\left(\mathrm{~s}, 6 \mathrm{H}, \mathrm{H}_{\mathrm{f}}\right) \mathrm{ppm} .{ }^{13} \mathbf{C}\left\{{ }^{1} \mathbf{H}\right\}-$ NMR $\left(\mathrm{CDCl}_{3}, 126 \mathrm{MHz}\right) \delta: 149.6,148.4,142.9,131.5,131.1,126.0,125.5,122.3,117.8$, 94.5, 86.2, 47.2, 27.3 ppm. MS(ASAP): $m / z 266.088[\mathrm{M}+\mathrm{H}]^{+}$.

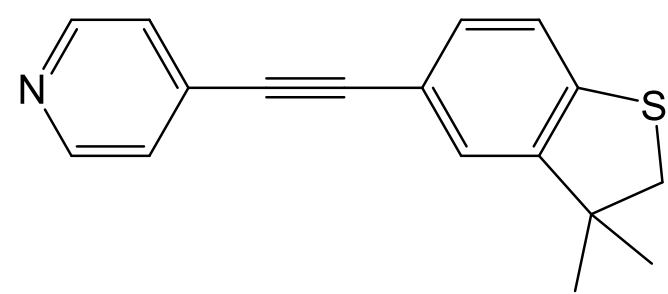

\section{1-(4-((3,3-dimethyl-2,3-dihydrobenzothiophen-5-yl)ethynyl)pyridin-1-ium-1-yl)-}

2,3,4-trioxocyclobutan-1-ide (3). A solution of squaric acid (400 mg, $3.44 \mathrm{mmol}$ ) in acetic anhydride $(30 \mathrm{~mL})$ was heated under reflux for $1 \mathrm{~h}$ before the solution was cooled to room temperature. $\mathbf{b}(911 \mathrm{mg}, 3.44 \mathrm{mmol})$ was added to the solution and then heated under reflux for $4 \mathrm{~h}$ forming a red solution. The solution was cooled to room temperature and the solvent was removed under vассио leaving a red oil. The residue was triturated with methanol and the precipitate was collected by filtration. Yield: $1.03 \mathrm{~g}(83 \%)$. ${ }^{\mathbf{1}} \mathbf{H}-\mathbf{N M R}\left(\mathrm{DMSO}_{\mathrm{d}}, 600\right.$ MHz) $\delta 9.23\left(\mathrm{~d},{ }^{3} J_{\mathrm{HH}}=7.18 \mathrm{~Hz}, 2 \mathrm{H}, \mathrm{H}_{\mathrm{e}}\right), 8.17\left(\mathrm{~d},{ }^{3} J_{\mathrm{HH}}=7.22 \mathrm{~Hz}, 2 \mathrm{H}, \mathrm{H}_{\mathrm{d}}\right), 7.48-7.46(\mathrm{~m}, 2 \mathrm{H}$, $\left.\mathrm{H}_{\mathrm{a}}+\mathrm{H}_{\mathrm{b}}\right), 7.35\left(\mathrm{~d},{ }^{3} J_{\mathrm{HH}}=8.00 \mathrm{~Hz}, 1 \mathrm{H}, \mathrm{H}_{\mathrm{c}}\right), 3.25\left(\mathrm{~s}, 2 \mathrm{H}, \mathrm{H}_{\mathrm{g}}\right), 1.33\left(\mathrm{~s}, 6 \mathrm{H}, \mathrm{H}_{\mathrm{f}}\right) \mathrm{ppm} .{ }^{13} \mathbf{C}\left\{{ }^{1} \mathbf{H}\right\}-$ NMR (DMSO-d 6 , 126 MHz) $\delta: 211.5,188.3,164.4,149.6,146.0,138.3,135.5,132.5,129.4$, 127.3, 123.2, 116.0, 105.7, 87.4, 47.3, 46.9, 27.3 ppm. MS(ASAP): $m / z, 362.078[\mathrm{M}+\mathrm{H}]^{+} .1$

\footnotetext{
${ }^{1}$ Elemental analysis consistently gave values consistent with the parent pyridine suggesting the ylide is not thermally stable.
} 


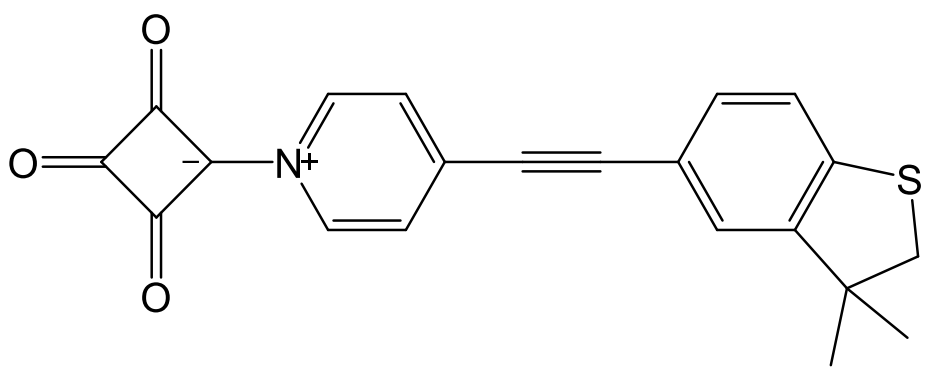

\section{3-(4-((3,3-dimethyl-2,3-dihydrobenzo[b]thiophen-5-yl)ethynyl)phenyl)-4-}

hydroxypent-3-en-2-one (4). $\mathrm{Pd}\left(\mathrm{PPh}_{3}\right)_{4}(300 \mathrm{mg}, 0.26 \mathrm{mmol})$ and $\mathrm{CuI}(49 \mathrm{mg}, 0.26 \mathrm{mmol})$ were added to a freeze-pump-thaw degassed solution containing 5-ethynyl-3,3-dimethyl-2,3dihydrobenzothiophene (500 mg, $2.65 \mathrm{mmol}$ ), 4-hydroxy-3-(4-iodophenyl)pent-3-en-2-one (800 mg, $2.65 \mathrm{mmol}), \mathrm{Et}_{3} \mathrm{~N}(5 \mathrm{~mL})$, and THF $(20 \mathrm{~mL})$. The solution was stirred for $16 \mathrm{~h}$ before the solvent was removed. Purification was achieved by column chromatography eluted by a solvent gradient from DCM:hexane (1:1) to neat DCM. Crystals were grown by evaporation of a DCM/MeOH solution. Yield: $0.25 \mathrm{~g}(30 \%) .{ }^{\mathbf{1}} \mathbf{H}-\mathbf{N M R}\left(\mathrm{CDCl}_{3}, 600 \mathrm{MHz}\right) \delta$ $7.53\left(\mathrm{~d},{ }^{3} J_{\mathrm{HH}}=8.3 \mathrm{~Hz}, 2 \mathrm{H}, \mathrm{H}_{\mathrm{e}}\right), 7.29\left(\mathrm{dd},{ }^{3} J_{\mathrm{HH}}=7.94 \mathrm{~Hz},{ }^{3} J_{\mathrm{HH}}=1.63 \mathrm{~Hz}, 1 \mathrm{H}, \mathrm{H}_{\mathrm{b}}\right), 7.19(\mathrm{~d}$, $\left.{ }^{4} J_{\mathrm{HH}}=1.6 \mathrm{~Hz}, 1 \mathrm{H}, \mathrm{H}_{\mathrm{a}}\right), 7.16-7.14\left(\mathrm{~m}, 3 \mathrm{H}, \mathrm{H}_{\mathrm{c}}+\mathrm{H}_{\mathrm{d}}\right), 3.20\left(\mathrm{~s}, 2 \mathrm{H}, \mathrm{H}_{\mathrm{h}}\right), 1.89\left(\mathrm{~s}, 6 \mathrm{H}, \mathrm{H}_{\mathrm{f}}\right), 1.39(\mathrm{~s}$, $\left.6 \mathrm{H}, \mathrm{H}_{\mathrm{g}}\right)$ ppm. ${ }^{13} \mathbf{C}\left\{{ }^{1} \mathbf{H}\right\}$-NMR $\left(\mathrm{CDCl}_{3}, 126 \mathrm{MHz}\right) \delta: 190.7,148.2,141.7,136.7,131.8,131.1$, 130.8, 125.8, 122.7, 122.2, 118.8, 114.7, 90.4, 88.2, 47.2, 27.3, $24.1 \mathrm{ppm}$. MS(ASAP): $m / z$ $363.124[\mathrm{M}+\mathrm{H}]^{+}{ }^{2}$

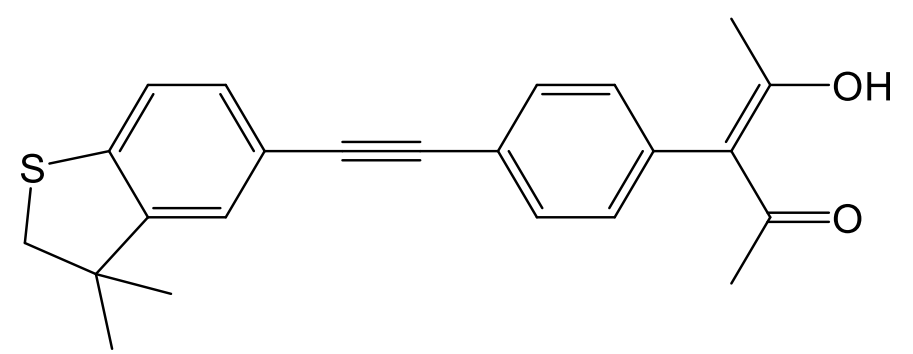

\section{3-hydroxy-1-(4-((4-(methylthio)phenyl)ethynyl)phenyl)but-2-en-1-one}

$\mathrm{Pd}\left(\mathrm{PPh}_{3}\right)_{4}(381 \mathrm{mg}, 0.33 \mathrm{mmol})$ and $\mathrm{CuI}(62 \mathrm{mg}, 0.33 \mathrm{mmol})$ were added to a freeze-pumpthaw degassed solution containing (4-ethynylphenyl)(methyl)sulfane (500 mg, $3.37 \mathrm{mmol}$ ), 3-hydroxy-1-(4-iodophenyl)but-2-en-1-one (967 mg, $3.37 \mathrm{mmol}), \mathrm{Et}_{3} \mathrm{~N}$ (5 mL), and THF (20 $\mathrm{mL}$ ). The solution was stirred for $16 \mathrm{~h}$ before the solvent was removed. Purification was achieved by column chromatography eluted by a solvent gradient from DCM:hexane (1:1) to neat DCM providing a white solid. Yield: $0.75 \mathrm{~g}(72 \%) .{ }^{1} \mathbf{H}-\mathbf{N M R}\left(\mathrm{CDCl}_{3}, 600 \mathrm{MHz}\right) \delta 7.85$ $\left(\mathrm{d},{ }^{3} J_{\mathrm{HH}}=8.5 \mathrm{~Hz}, 2 \mathrm{H}, \mathrm{H}_{\mathrm{d}}\right), 7.58\left(\mathrm{~d},{ }^{3} J_{\mathrm{HH}}=8.5 \mathrm{~Hz}, 2 \mathrm{H}, \mathrm{H}_{\mathrm{c}}\right), 7.45\left(\mathrm{~d},{ }^{3} J_{\mathrm{HH}}=8.3 \mathrm{~Hz}, 2 \mathrm{H}, \mathrm{H}_{\mathrm{a}}\right)$,

\footnotetext{
${ }^{2}$ Repeated attempts were made to obtain elemental data but satisfactory results could not be obtained.
} 
$7.22\left(\mathrm{~d},{ }^{3} J_{\mathrm{HH}}=8.3 \mathrm{~Hz}, 2 \mathrm{H}, \mathrm{H}_{\mathrm{b}}\right), 6.18\left(\mathrm{~s}, 1 \mathrm{H}, \mathrm{H}_{\mathrm{e}}\right), 2.50\left(\mathrm{~s}, 3 \mathrm{H}, \mathrm{H}_{\mathrm{f}}\right), 2.21\left(\mathrm{~s}, 3 \mathrm{H}, \mathrm{H}_{\mathrm{g}}\right) \mathrm{ppm}$. ${ }^{13} \mathbf{C}\left\{{ }^{1} \mathbf{H}\right\}$-NMR $\left(\mathrm{CDCl}_{3}, 126 \mathrm{MHz}\right) \delta: 194.1,181.9,140.0,134.1,131.9,131.6,127.3,126.9$, 125.7, 118.9, 96.8, 92.2, 88.9, 25.9, 15.2 ppm. MS(ASAP): $m / z 309.100[\mathrm{M}+\mathrm{H}]^{+}$. Anal. Calc. for. $\mathrm{C}_{19} \mathrm{H}_{16} \mathrm{O}_{2} \mathrm{~S}: \mathrm{C}, 74.00 ; \mathrm{H}, 5.23 \%$ Found: $\mathrm{C}, 73.82 ; \mathrm{H}, 5.16 \%$

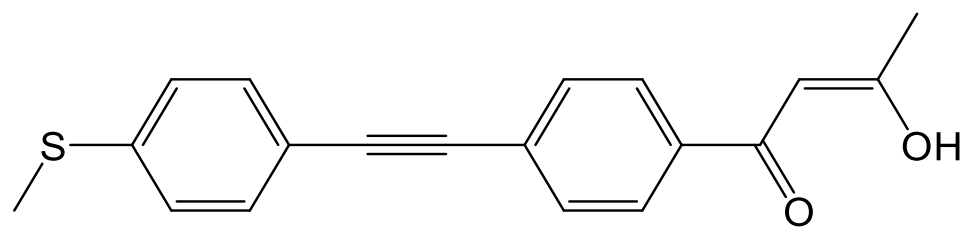

tert-butyl 3-(4-bromophenyl)-2-cyanoacrylate (c): 4-bromobenzaldehyde (8.18 g, $44.2 \mathrm{mmol})$ and 2,2,6,6-tetramethylpiperidine $(7.47 \mathrm{~g}, 8.92 \mathrm{~mL}, 53.0 \mathrm{mmol})$ were added to a solution of tert-butyl 2-cyanoacetate $(6.23 \mathrm{~g}, 44.2 \mathrm{mmol})$ in ethanol $(30 \mathrm{~mL})$. The reaction mixture was heated at reflux for $6 \mathrm{~h}$, then cooled to $0^{\circ} \mathrm{C}$ forming white crystals that were collected by filtration. Yield: $5.80 \mathrm{~g}(42 \%)$. ${ }^{1} \mathrm{H}-\mathbf{N M R}\left(\mathrm{CDCl}_{3}, 600 \mathrm{MHz}\right) \delta_{\mathrm{H}} 8.08(\mathrm{~s}, 1 \mathrm{H}$, $\left.\mathrm{H}_{\mathrm{c}}\right), 7.82\left(\mathrm{~d},{ }^{3} J_{\mathrm{HH}}=8.6 \mathrm{~Hz}, 2 \mathrm{H}, \mathrm{H}_{\mathrm{a}}\right), 7.62\left(\mathrm{~d},{ }^{3} J_{\mathrm{HH}}=8.6 \mathrm{~Hz}, 2 \mathrm{H}, \mathrm{H}_{\mathrm{b}}\right), 1.57\left(\mathrm{~s}, 9 \mathrm{H}, \mathrm{H}_{\mathrm{d}}\right) \mathrm{ppm}$. ${ }^{13} \mathbf{C}\left\{{ }^{1} \mathbf{H}\right\}$-NMR $\left(\mathrm{CDCl}_{3}, 126 \mathrm{MHz}\right) \delta_{\mathrm{C}} 160.9,152.2,132.5,132.0,130.3,127.8,115.4,105.2$,

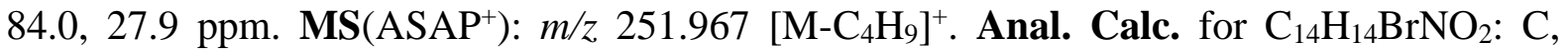
54.56; H, 4.58; N, $4.55 \%$. Found: C, 54.47; H, 4.57; N, $4.50 \%$.

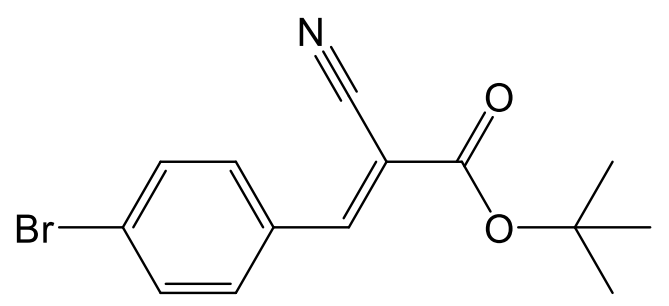

tert-butyl

2-cyano-3-(4-((trimethylsilyl)ethynyl)phenyl)acrylate

(d):

trimethylsilyacetylene $(2.27 \mathrm{~mL}, 1.59 \mathrm{~g}, 16.28 \mathrm{mmol})$ was added to a solution containing $\mathrm{PdCl}_{2}\left(\mathrm{PPh}_{3}\right)_{2}(1.19 \mathrm{~g}, 1.60 \mathrm{mmol}), \mathrm{CuI}(304 \mathrm{mg}, 1.60 \mathrm{mmol}), \mathbf{c}(5.0 \mathrm{~g}, 16.28 \mathrm{mmol}), \mathrm{Et}_{3} \mathrm{~N}$ (10 $\mathrm{mL})$ and THF $(100 \mathrm{~mL})$ that had been degassed by three freeze-pump-thaw cycles. The solution was heated to reflux for $16 \mathrm{~h}$ before the solvent was removed, leaving a brown oil. Purification was achieved by column chromatography on silica eluted by neat DCM to give a pale-yellow solid that solidified upon standing. Yield: $4.81 \mathrm{~g},(91 \%) .{ }^{\mathbf{1}} \mathbf{H}-\mathbf{N M R}\left(\mathrm{CDCl}_{3}, 600\right.$ MHz) $\delta_{\mathrm{H}} 8.09\left(\mathrm{~s}, 1 \mathrm{H}, \mathrm{H}_{\mathrm{c}}\right), 7.89\left(\mathrm{~d},{ }^{3} J_{\mathrm{HH}}=8.1 \mathrm{~Hz}, 2 \mathrm{H}, \mathrm{H}_{\mathrm{a}}\right), 7.53\left(\mathrm{~d},{ }^{3} J_{\mathrm{HH}}=8.1 \mathrm{~Hz}, 2 \mathrm{H}, \mathrm{H}_{\mathrm{b}}\right)$, $1.56\left(\mathrm{~s}, 9 \mathrm{H}, \mathrm{H}_{\mathrm{d}}\right), 0.24\left(\mathrm{~s}, 9 \mathrm{H}, \mathrm{H}_{\mathrm{e}}\right)$ ppm. ${ }^{13} \mathbf{C}\left\{{ }^{1} \mathbf{H}\right\}-\mathbf{N M R}\left(\mathrm{CDCl}_{3}, 126 \mathrm{MHz}\right) \delta_{\mathrm{C}} 161.0,152.7$, 
$132.5,131.2,130.6,127.8,115.5,104.9,103.9,99.0,83.8,27.9,-0.23$ ppm. MS(ASAP $\left.{ }^{+}\right)$: $m / z 270.091\left[\mathrm{M}-\mathrm{C}_{4} \mathrm{H}_{9}\right]^{+}$. Anal. Calc. for $\mathrm{C}_{19} \mathrm{H}_{23} \mathrm{NO}_{2} \mathrm{Si}$ : C, 70.11; H, 7.12; N, $4.30 \%$. Found: C, $70.13 ; \mathrm{H}, 7.14 ; \mathrm{N}, 4.00 \%$.

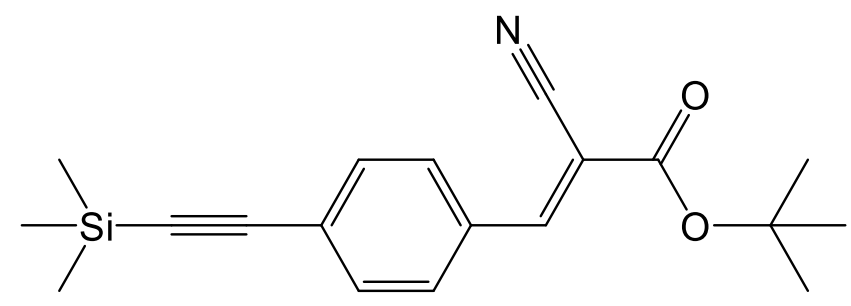

tert-butyl 2-cyano-3-(4-ethynylphenyl)acrylate (e): potassium fluoride (1.39 g, 24.0 mmol) was added to the solution containing $\mathbf{d}(4.0 \mathrm{~g}, 12.3)$, methanol (25 mL) and THF (25 $\mathrm{mL}$ ). The solution was stirred at room temperature for $6 \mathrm{~h}$, the solvent was removed under vaccuo. The residue was dissolved in DCM, filtered and solvent removed from the collected filtrate under vaccuo leaving a brown solid. Yield: $2.80 \mathrm{~g},(90 \%) . \quad{ }^{\mathbf{1}} \mathbf{H}-\mathbf{N M R}\left(\mathrm{CDCl}_{3}, 600\right.$ $\mathrm{MHz}): \delta_{\mathrm{H}} 8.11\left(\mathrm{~s}, 1 \mathrm{H}, \mathrm{H}_{\mathrm{c}}\right), 7.91\left(\mathrm{~d},{ }^{3} J_{\mathrm{HH}}=8.0 \mathrm{~Hz}, 2 \mathrm{H}, \mathrm{H}_{\mathrm{a}}\right), 7.57\left(\mathrm{~d},{ }^{3} J_{\mathrm{HH}}=8.1 \mathrm{~Hz}, 2 \mathrm{H}, \mathrm{H}_{\mathrm{b}}\right)$, $3.27\left(\mathrm{~s}, 1 \mathrm{H}, \mathrm{H}_{\mathrm{e}}\right), 1.57\left(\mathrm{~s}, 9 \mathrm{H}, \mathrm{H}_{\mathrm{d}}\right) \mathrm{ppm} .{ }^{13} \mathbf{C}\left\{{ }^{1} \mathbf{H}\right\}-\mathbf{N M R}\left(\mathrm{CDCl}_{3}, 126 \mathrm{MHz}\right): \delta_{\mathrm{C}} 161.1,152.6$, 132.7, 131.6, 130.6, 126.7, 115.4, 105.4, 84.0, 82.6, 80.9, 27.9 ppm. MS(ASAP $\left.{ }^{+}\right): m / z$ $198.057\left[\mathrm{M}-\mathrm{C}_{4} \mathrm{H}_{8}\right]^{+}{ }^{3}$

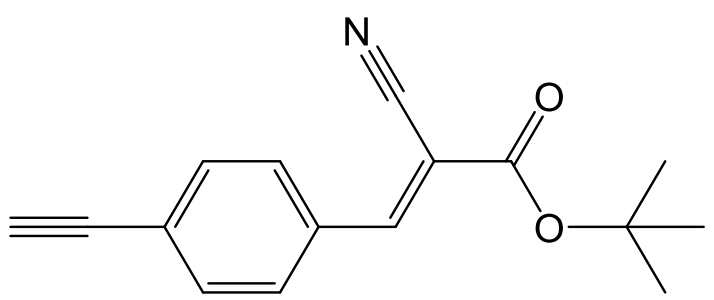

tert-butyl 3-(4-((4-(acetylthio)phenyl)ethynyl)phenyl)-2-cyanoacrylate (f): $\operatorname{Pd}\left(\mathrm{PPh}_{3}\right)_{4}$ (231 mg, $0.20 \mathrm{mmol})$ and $\mathrm{CuI}(38 \mathrm{mg}, 0.20 \mathrm{mmol})$ were added to a freeze-pump-thaw degassed solution containing e $(500 \mathrm{mg}, 1.97 \mathrm{mmol})$, S-(4-iodophenyl) ethanethioate (554 $\mathrm{mg}, 2.00 \mathrm{mmol}), \mathrm{Et}_{3} \mathrm{~N}(5 \mathrm{~mL})$ and THF $(20 \mathrm{~mL})$. The solution was stirred for $16 \mathrm{~h}$ before the solvent was removed, leaving a yellow oil. Purification was achieved by column chromatography eluted by a solvent gradient from DCM:hexane $(1: 1)$ to neat DCM giving a pale-yellow solid. Yield: $0.438 \mathrm{~g},(55 \%) .{ }^{1} \mathbf{H}-\mathbf{N M R}\left(\mathrm{CDCl}_{3}, 600 \mathrm{MHz}\right) \delta_{\mathrm{H}} 8.41\left(\mathrm{~s}, 1 \mathrm{H}, \mathrm{H}_{\mathrm{c}}\right)$,

\footnotetext{
${ }^{3}$ Too unstable for elemental analysis
} 
$7.97\left(\mathrm{~d},{ }^{3} J_{\mathrm{HH}}=8.2 \mathrm{~Hz}, 2 \mathrm{H}, \mathrm{H}_{\mathrm{a}}\right), 7.62\left(\mathrm{~d},{ }^{3} J_{\mathrm{HH}}=8.2 \mathrm{~Hz}, 2 \mathrm{H}, \mathrm{H}_{\mathrm{b}}\right), 7.58\left(\mathrm{~d},{ }^{3} J_{\mathrm{HH}}=8.2 \mathrm{~Hz}, 2 \mathrm{H}\right.$, $\left.\mathrm{H}_{\mathrm{f}}\right), 7.42\left(\mathrm{~d},{ }^{3} J_{\mathrm{HH}}=8.2 \mathrm{~Hz}, 2 \mathrm{H}, \mathrm{H}_{\mathrm{e}}\right), 2.44\left(\mathrm{~s}, 3 \mathrm{H}, \mathrm{H}_{\mathrm{g}}\right), 1.59\left(\mathrm{~s}, 9 \mathrm{H}, \mathrm{H}_{\mathrm{d}}\right)$ ppm. ${ }^{13} \mathbf{C}\left\{{ }^{1} \mathbf{H}\right\}-\mathbf{N M R}$ $\left(\mathrm{CDCl}_{3}, 126 \mathrm{MHz}\right) \delta_{\mathrm{C}} 193.1,161.1,152.7,134.2,132.3,132.2,131.3,130.8,128.9,127.6$,

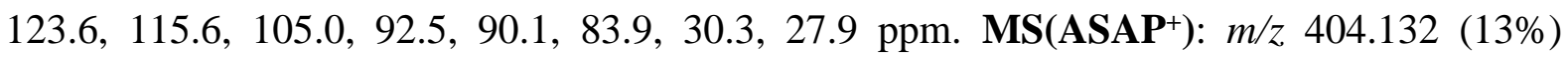
$[\mathrm{M}+\mathrm{H}]^{+}, \quad 404.132(13 \%) \quad\left[\mathrm{M}-\mathrm{C}_{2} \mathrm{H}_{2} \mathrm{O}\right]^{+}, 348.062(100 \%) \quad\left[\mathrm{M}-\mathrm{C}_{4} \mathrm{H}_{7}\right]^{+}$. Anal. Calc. for $\mathrm{C}_{24} \mathrm{H}_{21} \mathrm{NO}_{3} \mathrm{~S}: \mathrm{C}, 71.44 ; \mathrm{H}, 5.25 ; \mathrm{N}, 3.47 \%$. Found: C, 71.21; H, 5.19; N, $3.27 \%$.

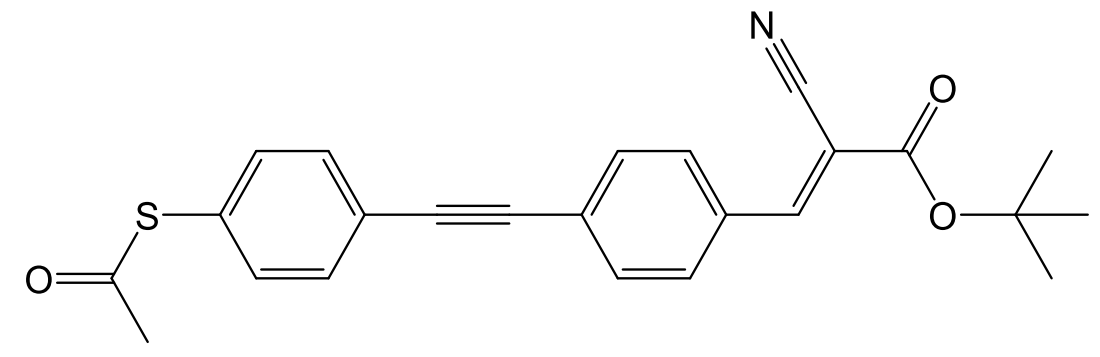

3-(4-((4-(acetylthio)phenyl)ethynyl)phenyl)-2-cyanoacrylic acid (7-acrylate): trifluoroacetic acid $(5 \mathrm{~mL})$ was added to a solution of $\mathbf{f}(200 \mathrm{mg}, 0.49 \mathrm{mmol})$ in DCM (30 $\mathrm{mL}$ ) and stirred at room temperature for $16 \mathrm{~h}$ before the solvent was removed. Methanol was added to the residue, sonicated forming to form a precipitate that was collected by filtration to give a pale-yellow solid. Yield: $90 \mathrm{mg},(52 \%) .{ }^{1} \mathbf{H}-\mathbf{N M R}\left(\mathrm{DMSO}_{6}, 600 \mathrm{MHz}\right) \delta_{\mathrm{H}} 8.34(\mathrm{~s}$, $\left.1 \mathrm{H}, \mathrm{H}_{\mathrm{c}}\right), 8.07\left(\mathrm{~d},{ }^{3} J_{\mathrm{HH}}=8.0 \mathrm{~Hz}, 2 \mathrm{H}, \mathrm{H}_{\mathrm{a}}\right), 7.75\left(\mathrm{~d},{ }^{3} J_{\mathrm{HH}}=8.0,2 \mathrm{H}, \mathrm{H}_{\mathrm{b}}\right), 7.65\left(\mathrm{~d},{ }^{3} J_{\mathrm{HH}}=8.1 \mathrm{~Hz}\right.$, $\left.2 \mathrm{H}, \mathrm{H}_{\mathrm{f}}\right), 7.48\left(\mathrm{~d},{ }^{3} \mathrm{~J}_{\mathrm{HH}}=8.0 \mathrm{~Hz}, 2 \mathrm{H}, \mathrm{H}_{\mathrm{e}}\right), 2.44\left(\mathrm{~s}, 3 \mathrm{H}, \mathrm{H}_{\mathrm{g}}\right) \mathrm{ppm} \cdot{ }^{13} \mathbf{C}\left\{{ }^{1} \mathbf{H}\right\}-\mathbf{N M R}\left(\mathrm{DMSO}-\mathrm{d}_{6}\right.$, $126 \mathrm{MHz}) \delta_{\mathrm{C}} 193.2,163.5,153.5,134.9,132.6,132.5,132.1,129.5,126.6,123.1,116.4$, 104.9, 92.4, 90.7, 30.7 ppm. MS(ASAP $\left.{ }^{+}\right): m / z \quad 348.065[\mathrm{M}+\mathrm{H}]^{+}$. Anal. Calc. for $\mathrm{C}_{20} \mathrm{H}_{13} \mathrm{NO}_{3} \mathrm{~S} \cdot 3 / 4 \mathrm{H}_{2} \mathrm{O}: \mathrm{C}, 66.56 ; \mathrm{H}, 4.05 ; \mathrm{N}, 3.88 \%$. Found: $\mathrm{C}, 66.35 ; \mathrm{H}, 3.65 ; \mathrm{N}, 3.83 \%$.

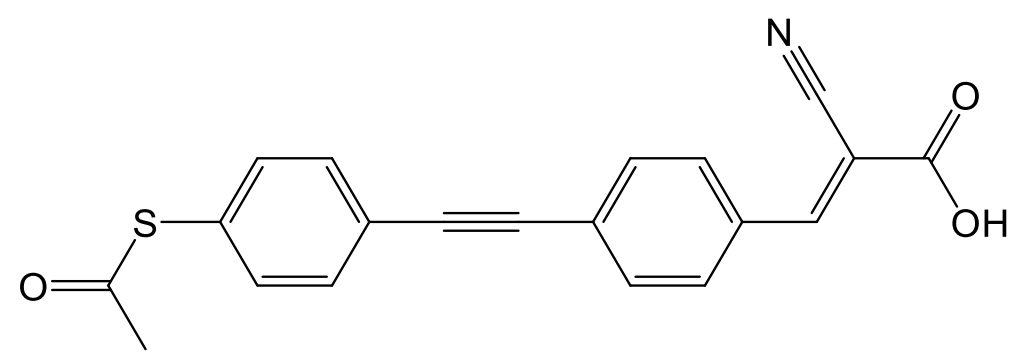

1-(4-((4-(acetylthio)phenyl)ethynyl)pyridin-1-ium-1-yl)-2,3,4-trioxocyclobutan-1-ide

(8-squarate): a solution of squaric acid $(400 \mathrm{mg}, 3.44 \mathrm{mmol})$ in acetic anhydride $(30 \mathrm{~mL})$ was heated to reflux for $1 \mathrm{~h}$ before the solution was cooled to room temperature. S-(4(pyridin-4-ylethynyl)phenyl) ethanethioate $(872 \mathrm{mg}, 3.44 \mathrm{mmol})$ was added to the solution, then heated to reflux for $4 \mathrm{~h}$ giving a red solution. The solution was cooled to room 
temperature, the solvent removed under vaccuo to give a red oil. The residue was triturated with methanol to give a red precipitate that was collected by filtration. Yield: $1.03 \mathrm{~g}(86 \%)$. ${ }^{1} \mathbf{H}-\mathbf{N M R}\left(\mathrm{CDCl}_{3}, 600 \mathrm{MHz}\right) \delta_{\mathrm{H}} 9.51\left(\mathrm{~d},{ }^{3} \mathrm{~J}_{\mathrm{HH}}=6.8 \mathrm{~Hz}, 2 \mathrm{H}, \mathrm{H}_{\mathrm{a}}\right), 7.96\left(\mathrm{~d},{ }^{3} \mathrm{~J}_{\mathrm{HH}}=7.3 \mathrm{~Hz}, 2 \mathrm{H}\right.$, $\left.\mathrm{H}_{\mathrm{b}}\right), 7.66\left(\mathrm{~d},{ }^{3} J_{\mathrm{HH}}=8.4 \mathrm{~Hz}, 2 \mathrm{H}, \mathrm{H}_{\mathrm{d}}\right), 7.51\left(\mathrm{~d},{ }^{3} J_{\mathrm{HH}}=8.2 \mathrm{~Hz}, 2 \mathrm{H}, \mathrm{H}_{\mathrm{c}}\right), 2.47\left(\mathrm{~s}, 3 \mathrm{H}, \mathrm{H}_{\mathrm{e}}\right) \mathrm{ppm}$. ${ }^{13} \mathbf{C}\left\{{ }^{1} \mathbf{H}\right\}$-NMR $\left(\mathrm{CDCl}_{3}, 126 \mathrm{MHz}\right) \delta_{\mathrm{C}} 208.5,192.2,189.2,138.6,134.4,133.8,133.10,132.5$, 129.2, 120.6, 109.9, 105.9, 87.0, 30.4 ppm. MS(ASAP+): $m / z 350.047(36.75 \%)[\mathrm{M}+\mathrm{H}]^{+}$, $254.065(100 \%)\left[\mathrm{M}-\mathrm{C}_{4} \mathrm{O}_{3}\right]^{+}$. Anal. Calc. for $\mathrm{C}_{19} \mathrm{H}_{11} \mathrm{NO}_{4} \mathrm{~S} \cdot 3 / 4 \mathrm{H}_{2} \mathrm{O}: \mathrm{C}, 62.58 ; \mathrm{H}, 3.47 ; \mathrm{N}$, 4.02\%. Found: C, 62.89; H, 3.47; N, 3.86\%.

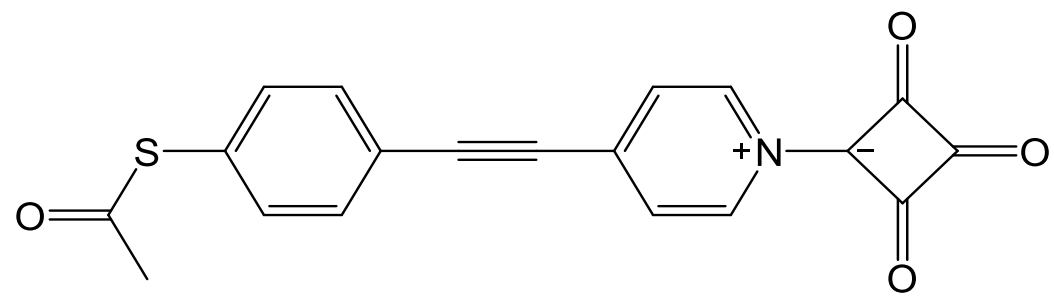

2,3,4-trioxo-1-(4-(phenylethynyl)pyridin-1-ium-1-yl)cyclobutan-1-ide (9): a solution of squaric acid (400 mg, $3.44 \mathrm{mmol})$ in acetic anhydride $(30 \mathrm{~mL})$ was heated to reflux for $1 \mathrm{~h}$, cooled to room temperature before 4-(phenylethynyl)pyridine $(615 \mathrm{mg}, 3.44 \mathrm{mmol})$ was added to the solution, heated to reflux for $4 \mathrm{~h}$ to give a red solution. The solution was cooled to room temperature, the solvent removed under vaccuo to give a red oil, which was triturated with methanol to give a precipitate that was collected by filtration. Yield: $0.85 \mathrm{~g}(90 \%) .{ }^{\mathbf{1}} \mathbf{H}$ NMR (DMSO-d $6,600 \mathrm{MHz}) \delta 9.28\left(\mathrm{~d},{ }^{3} \mathrm{~J}_{\mathrm{HH}}=6.8 \mathrm{~Hz}, 2 \mathrm{H}, \mathrm{H}_{\mathrm{a}}\right), 8.25\left(\mathrm{~d},{ }^{3} J_{\mathrm{HH}}=7.3 \mathrm{~Hz}, 2 \mathrm{H}\right.$, $\left.\mathrm{H}_{\mathrm{b}}\right), 7.74\left(\mathrm{~d},{ }^{3} J_{\mathrm{HH}}=8.1 \mathrm{~Hz}, 2 \mathrm{H}, \mathrm{H}_{\mathrm{c}}\right), 7.59-7.56\left(\mathrm{~m}, 1 \mathrm{H}, \mathrm{H}_{\mathrm{e}}\right), 7.53-7.51\left(\mathrm{~m}, 2 \mathrm{H}, \mathrm{H}_{\mathrm{d}}\right) \mathrm{ppm}$. ${ }^{13} \mathbf{C}\left\{{ }^{1} \mathbf{H}\right\}$-NMR (DMSO-d $\left.6126 \mathrm{MHz}\right) \delta: 211.6,188.3,164.3,138.1,135.7,133.0,131.7$, 129.9, 129.6, 120.3, 103.9, 86.9 ppm. MS(ASAP $\left.{ }^{+}\right): m / z 276.065[\mathrm{M}+\mathrm{H}]^{+}$. Anal. Calc. for $\mathrm{C}_{17} \mathrm{H}_{9} \mathrm{NO}_{3} \cdot 1 / 4 \mathrm{CH}_{4} \mathrm{O}: \mathrm{C}, 73.14 ; \mathrm{H}, 3.56 ; \mathrm{N}, 4.94 \%$. Found: C, 73.24; H, 3.24; N, 5.09\%.

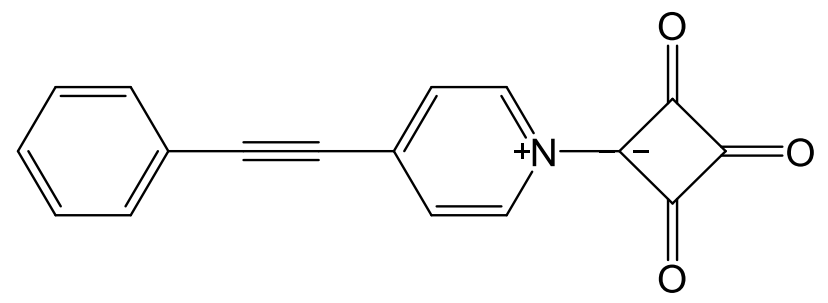


S2. NMR spectra of reported compounds

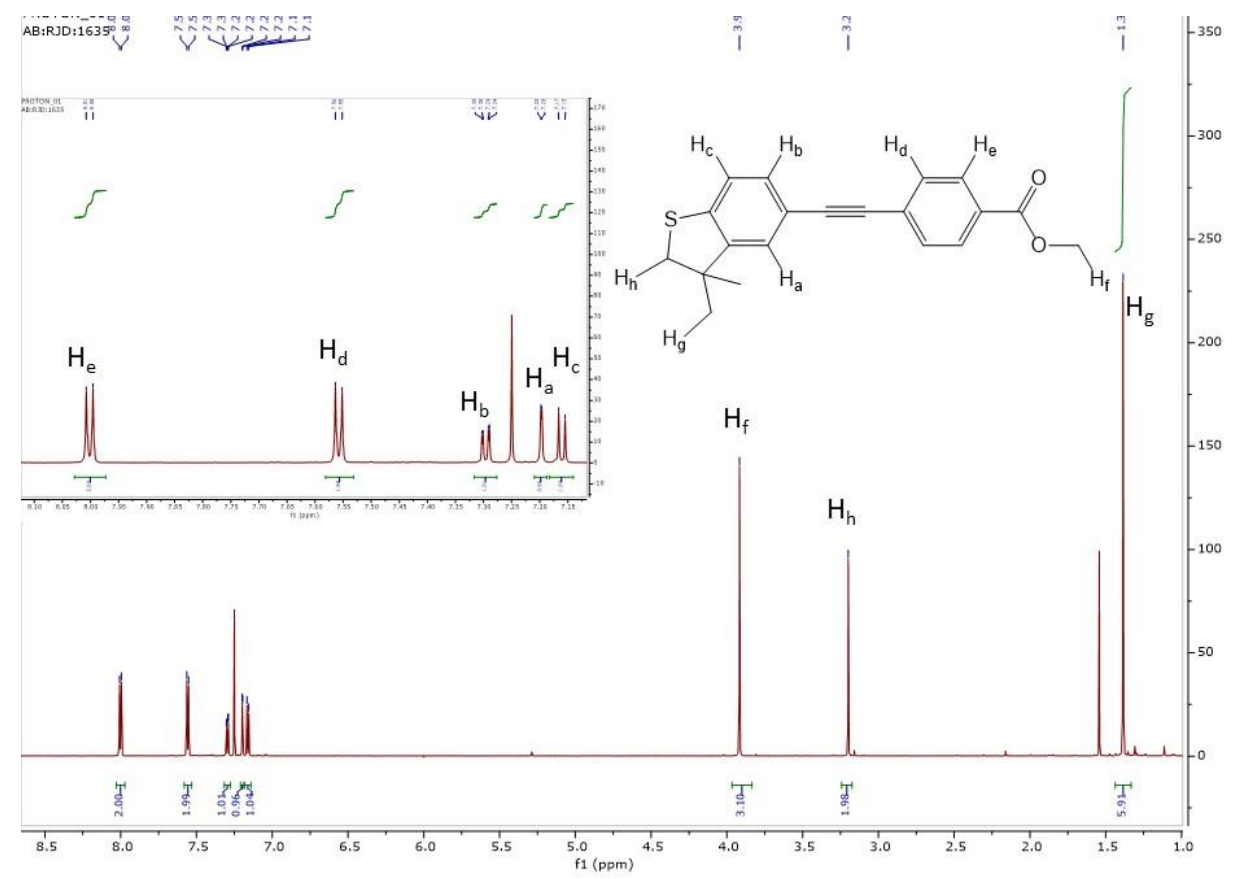

Figure $\mathrm{S}^{1} \mathrm{H} N \mathrm{NMR}$ of a recorded in $\mathrm{CDCl}_{3}$.

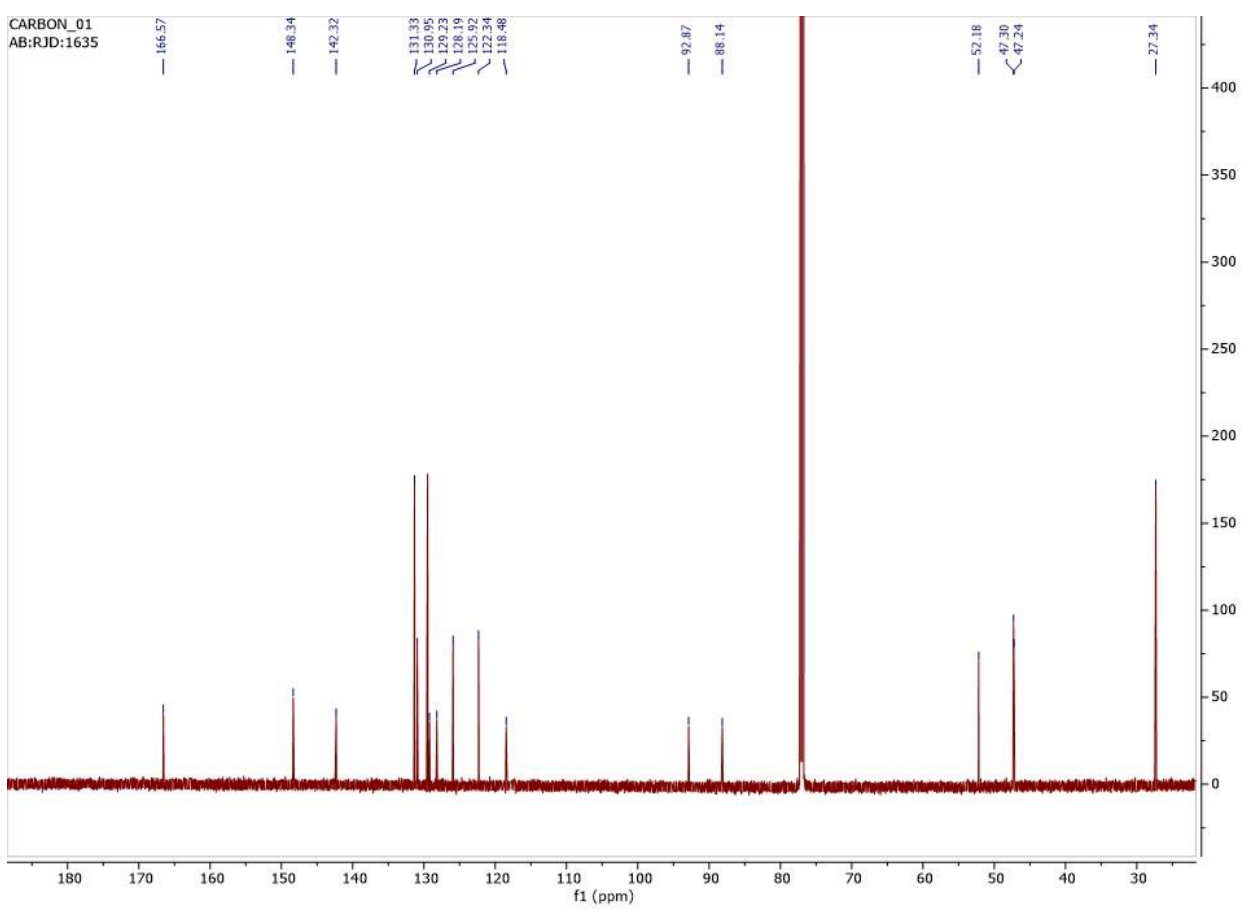

Figure S $2{ }^{13} \mathrm{C}$ NMR of a recorded in $\mathrm{CDCl}_{3}$. 


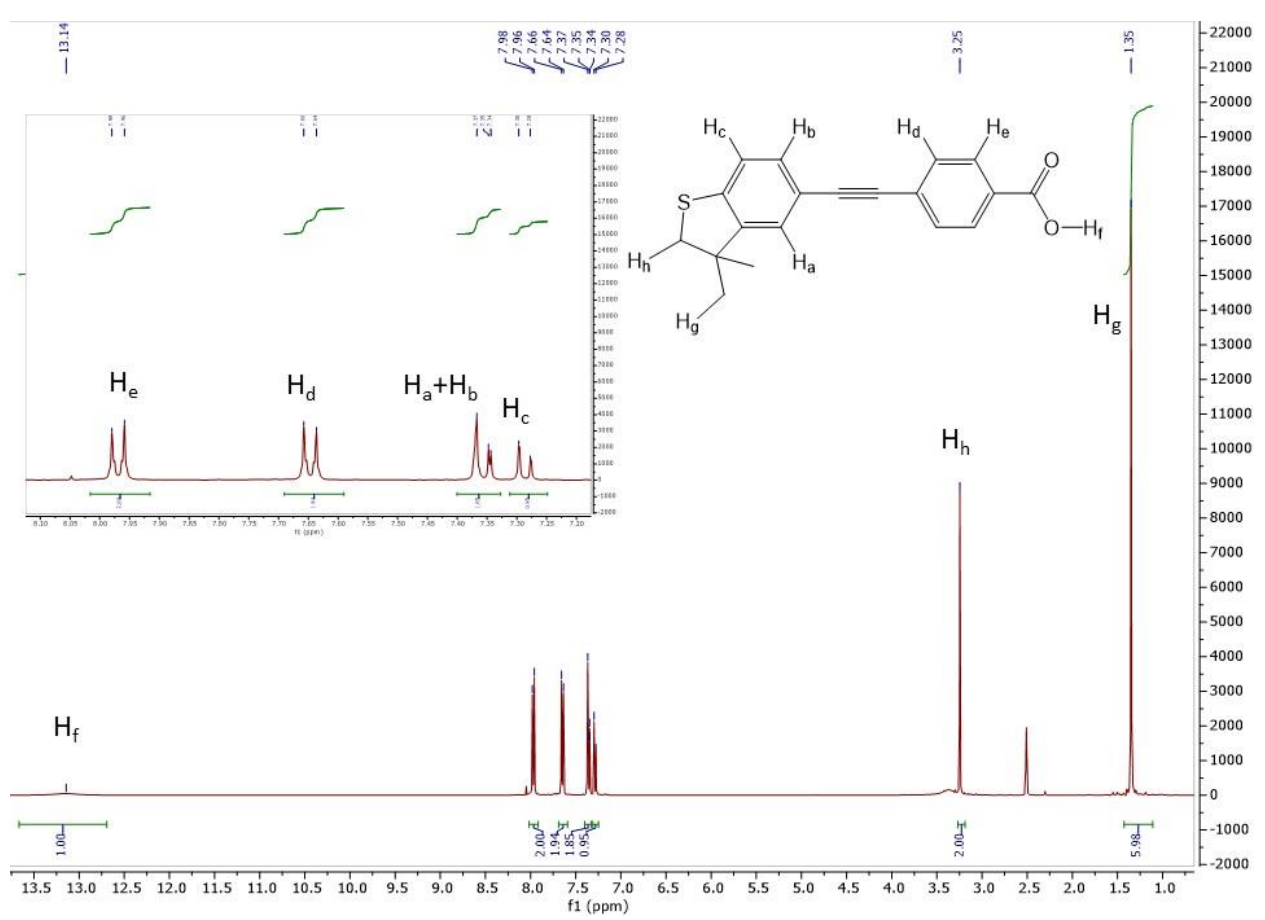

Figure $S 3^{1} \mathrm{H} N M R$ of 1 recorded in DMSO-d .

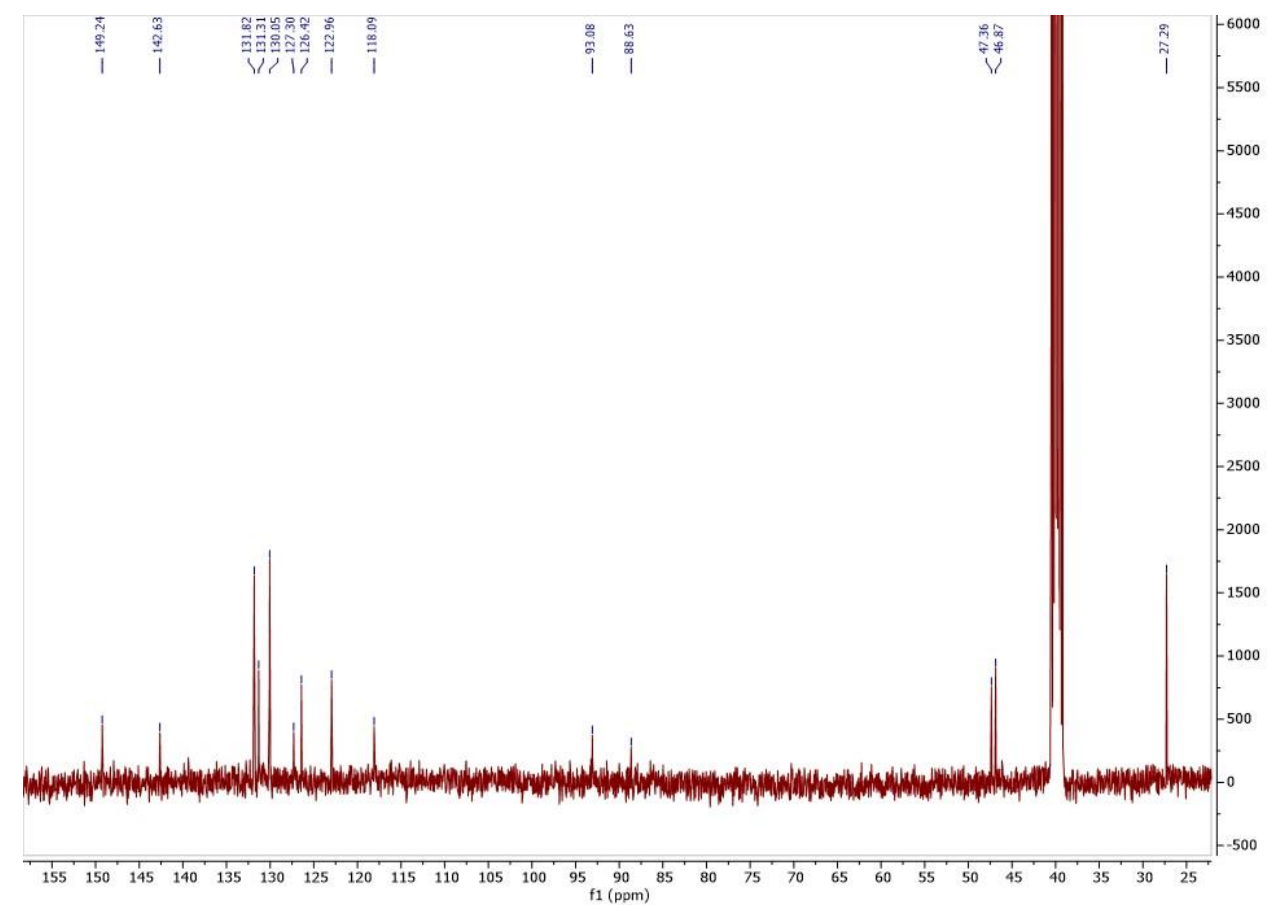

Figure $S 4{ }^{13} \mathrm{C} N \mathrm{NM}$ of 1 recorded in DMSO-d 


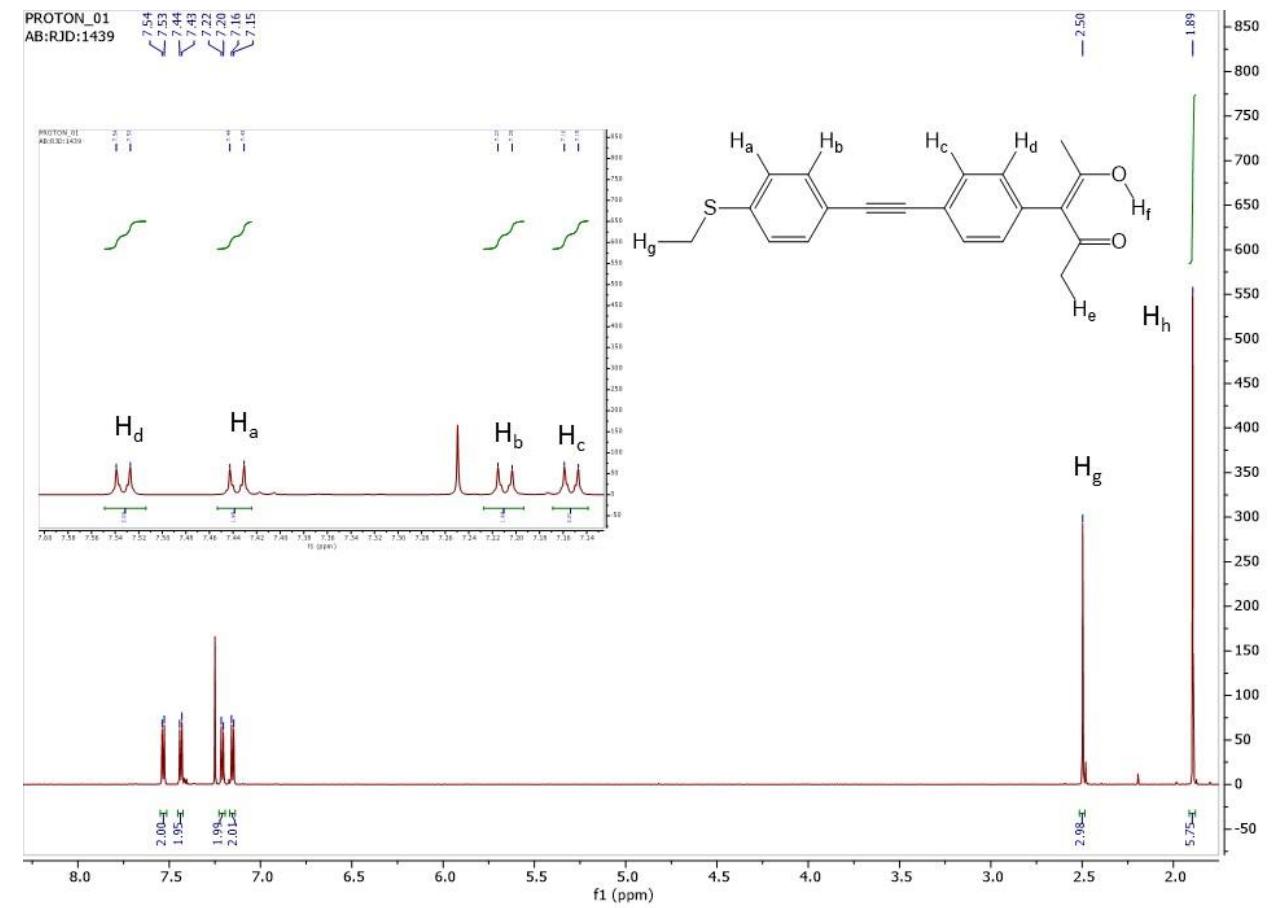

Figure $\mathrm{S}^{1} \mathrm{H} N \mathrm{NMR}$ of 2 recorded in $\mathrm{CDCl}_{3}$.

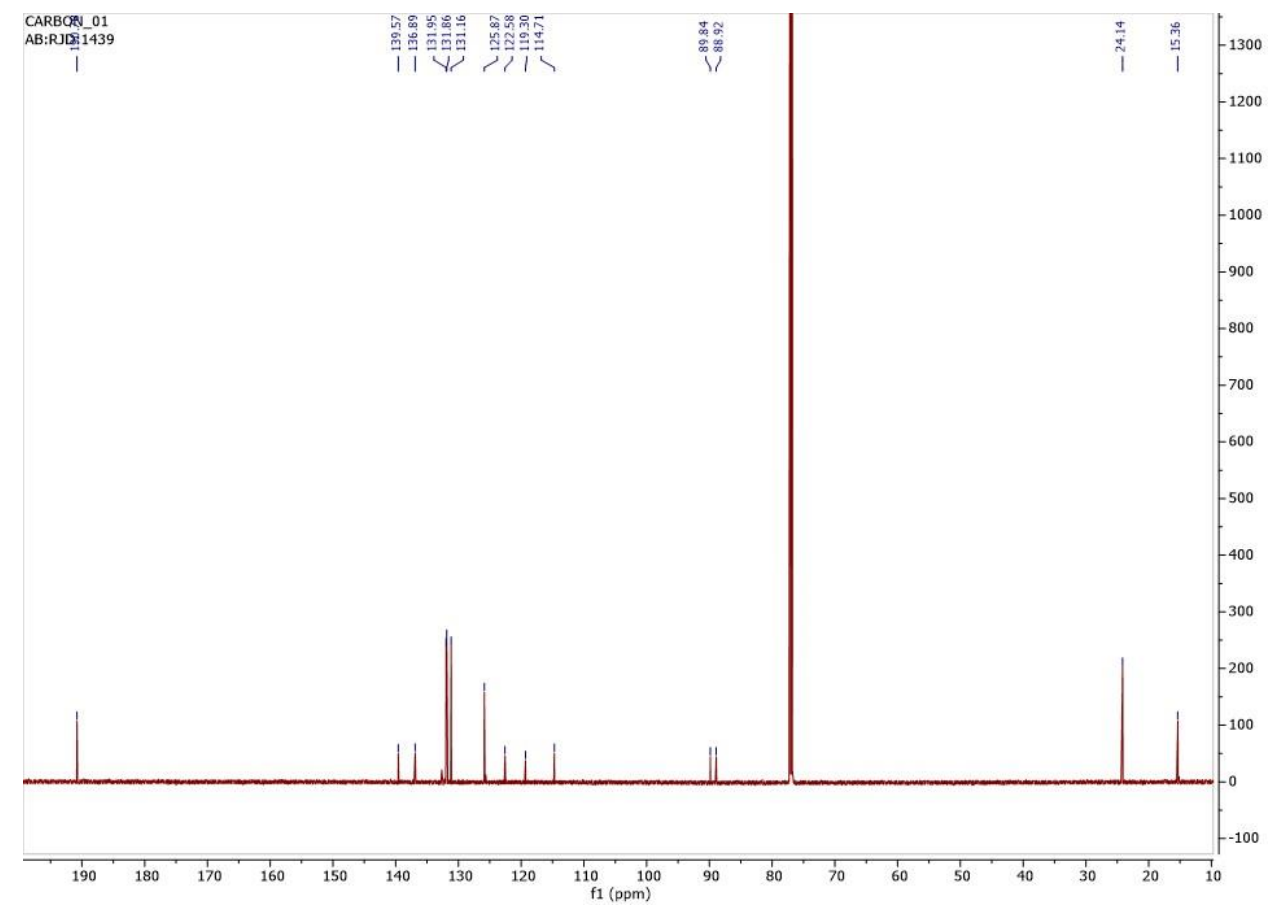

Figure $\mathrm{S}^{13} \mathrm{C}$ NMR of 2 recorded in $\mathrm{CDCl}_{3}$. 


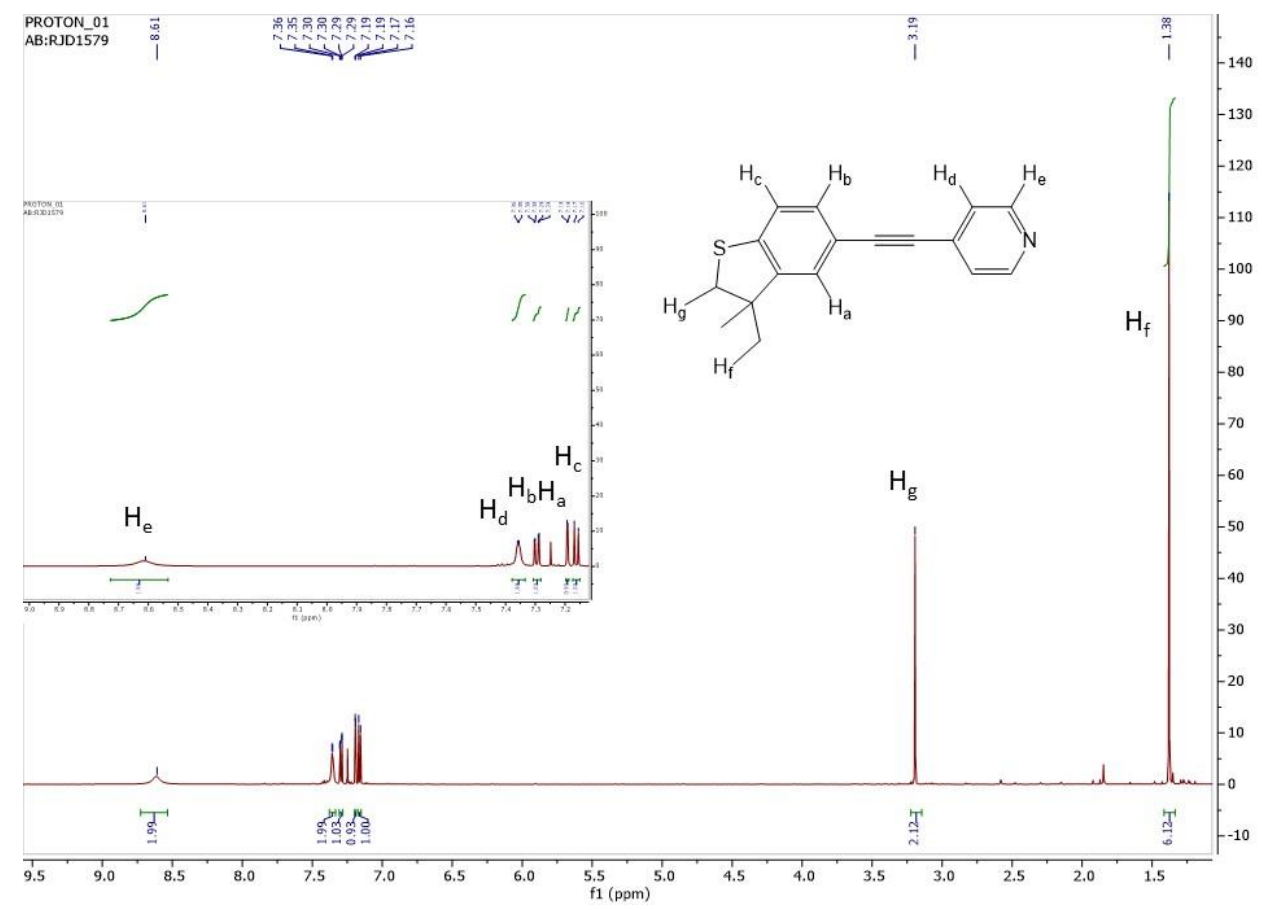

Figure $S 7^{1} \mathrm{H} N M R$ of b recorded in $\mathrm{CDCl}_{3}$.

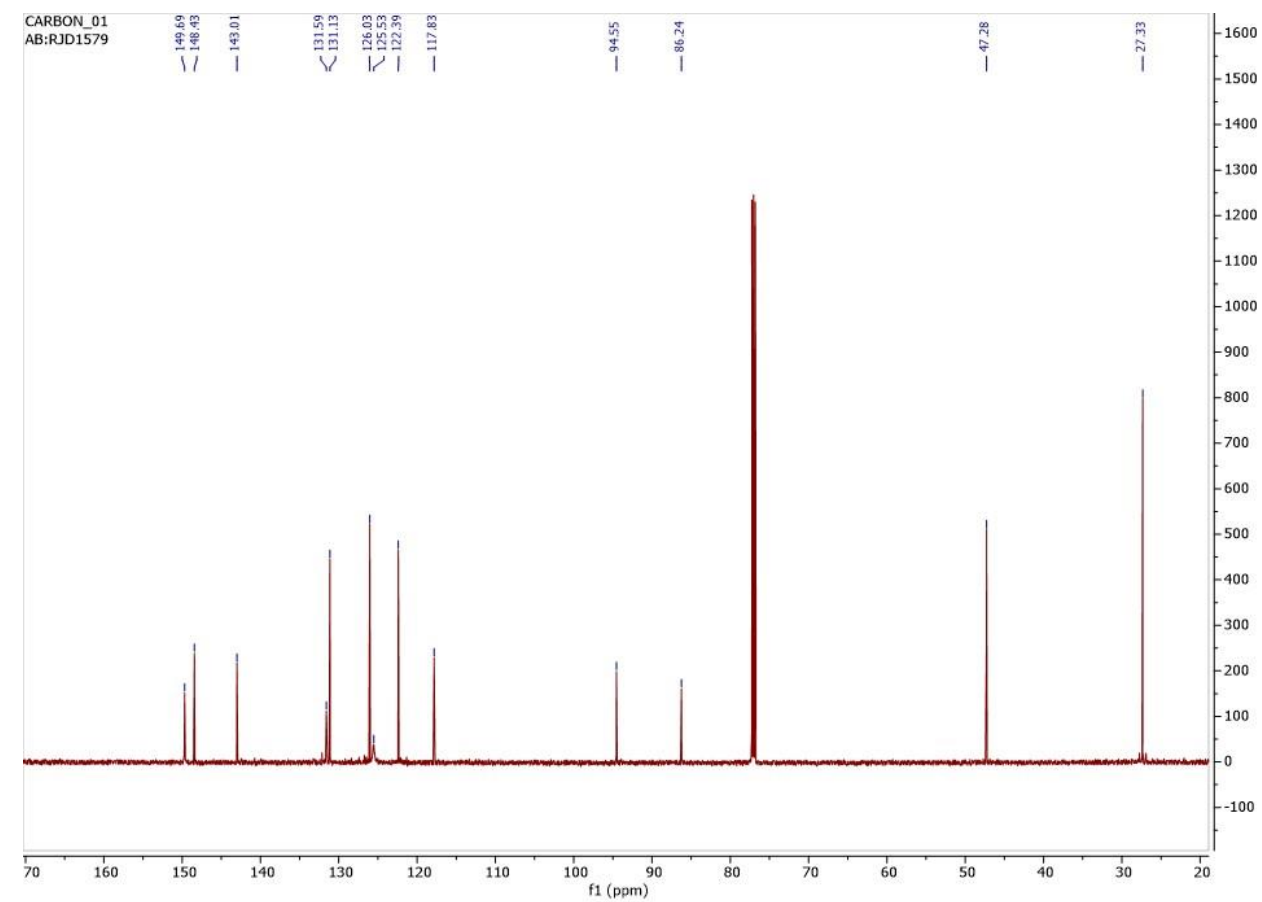

Figure $S 8^{13} \mathrm{C} N M R$ of b recorded in $\mathrm{CDCl}_{3}$. 


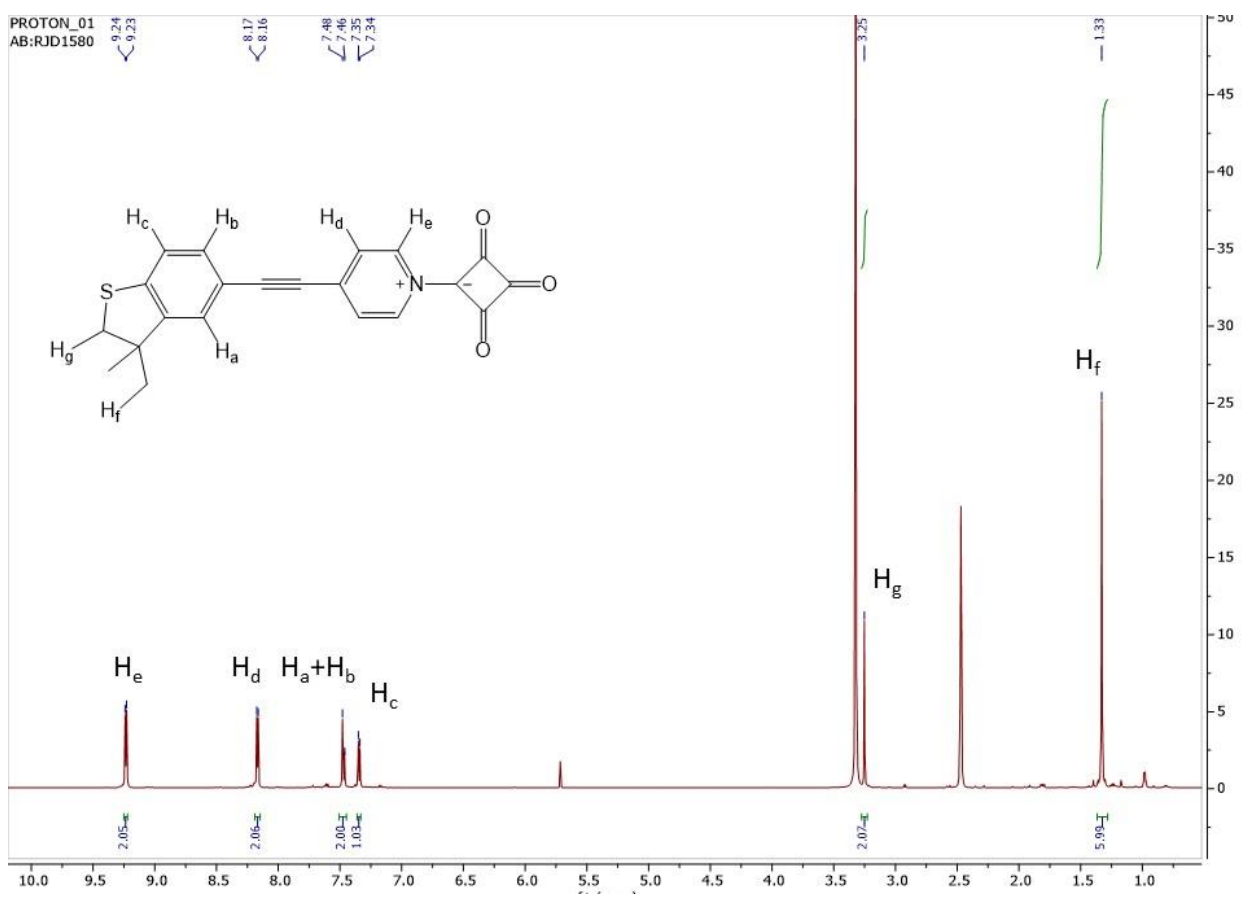

Figure $S 9{ }^{1} \mathrm{H} N M R$ of 3 recorded in DMSO-d

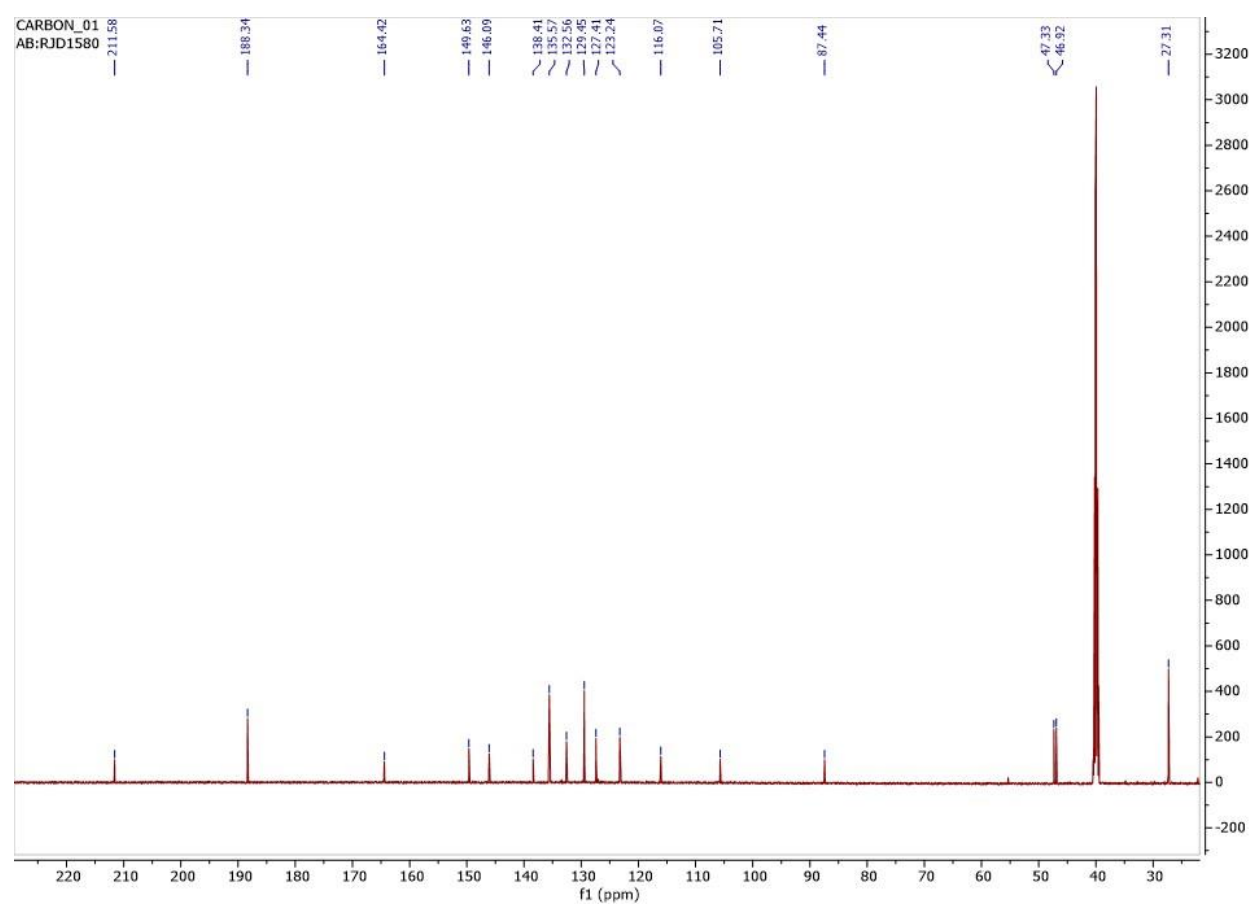

Figure $S 10^{13} \mathrm{C} N M R$ of 3 recorded in DMSO-d 


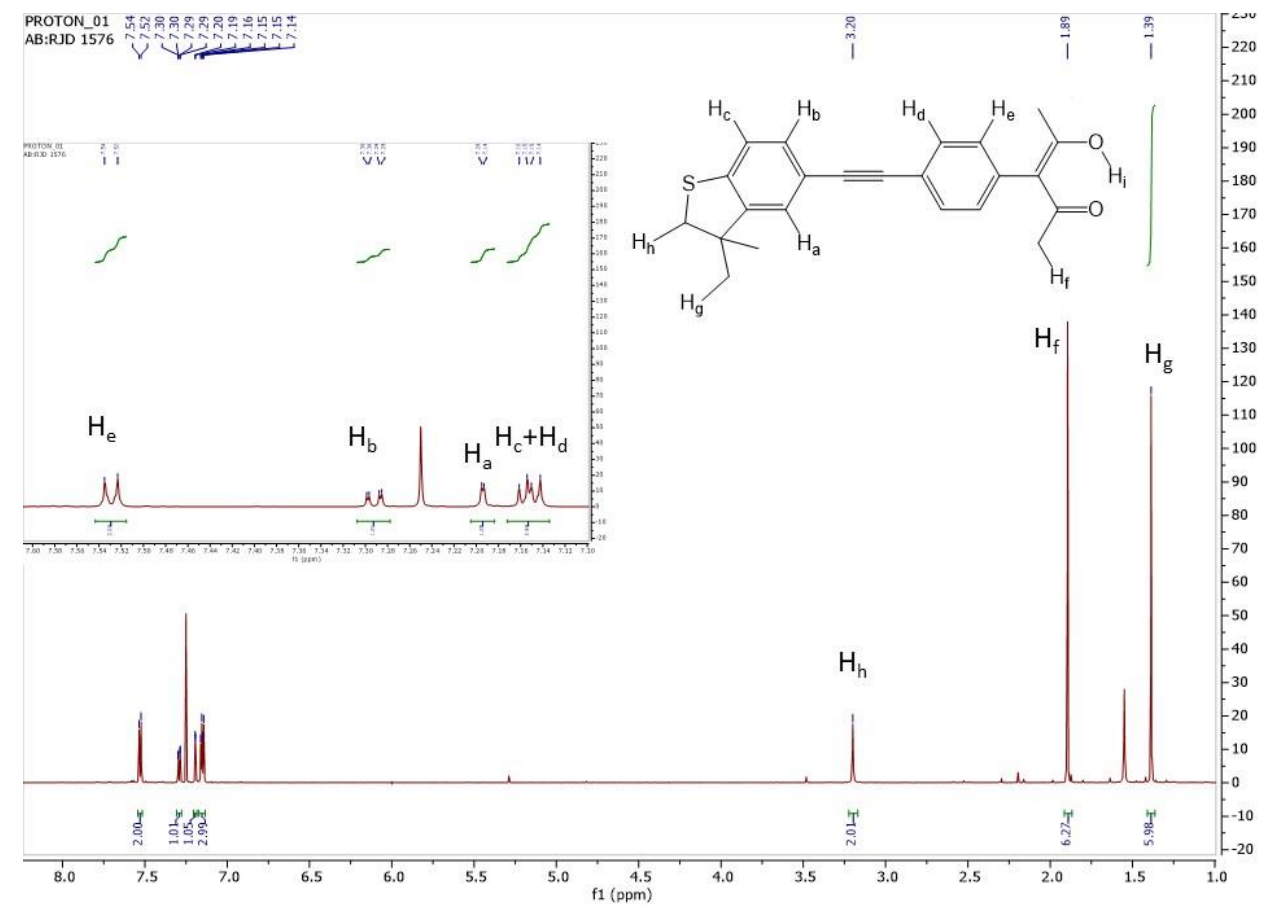

Figure $\mathrm{S}_{11}^{1} \mathrm{H} \mathrm{NMR}$ of 4 recorded in $\mathrm{CDCl}_{3}$

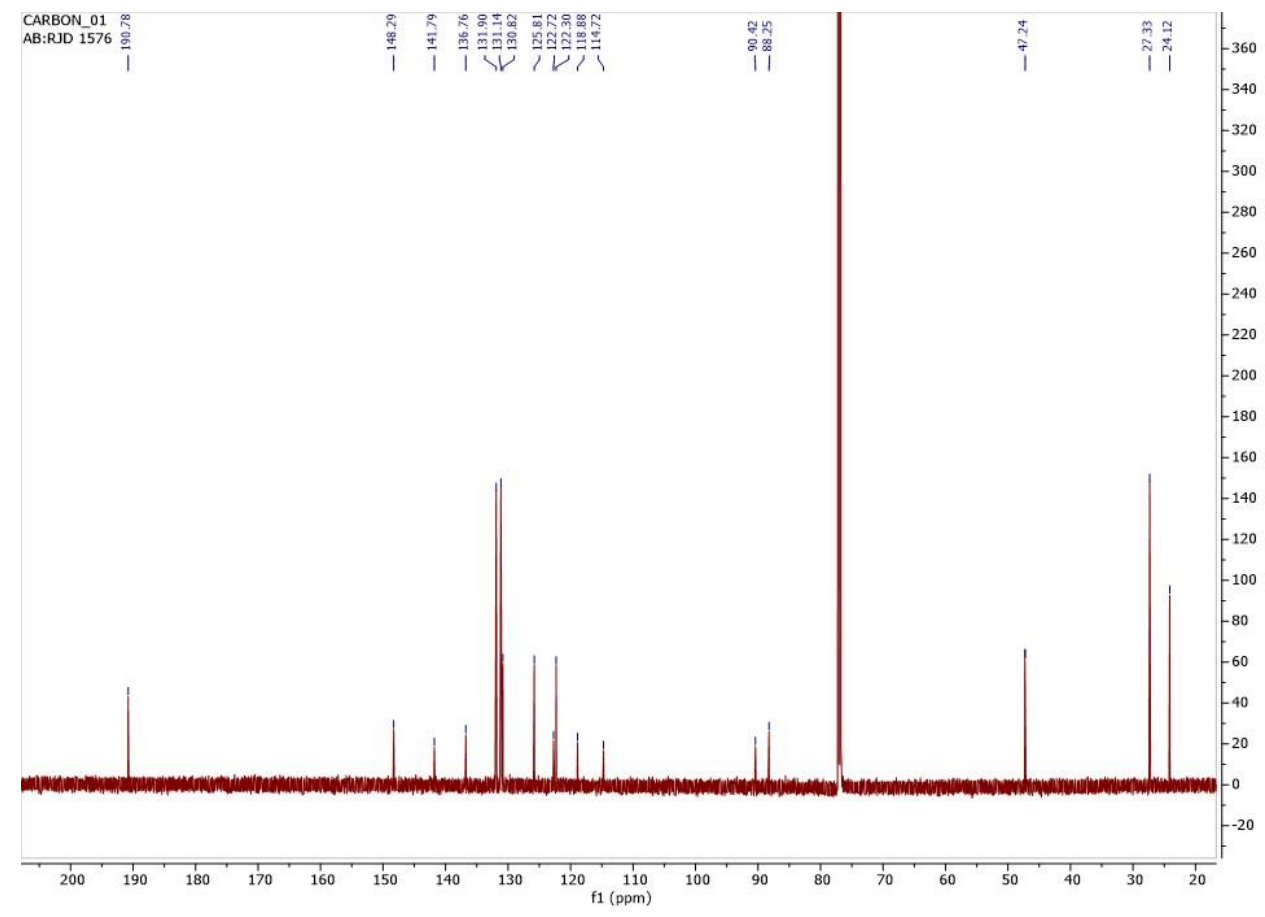

Figure $\mathrm{S}_{12}{ }^{13} \mathrm{C}$ NMR of 4 recorded in $\mathrm{CDCl}_{3}$ 


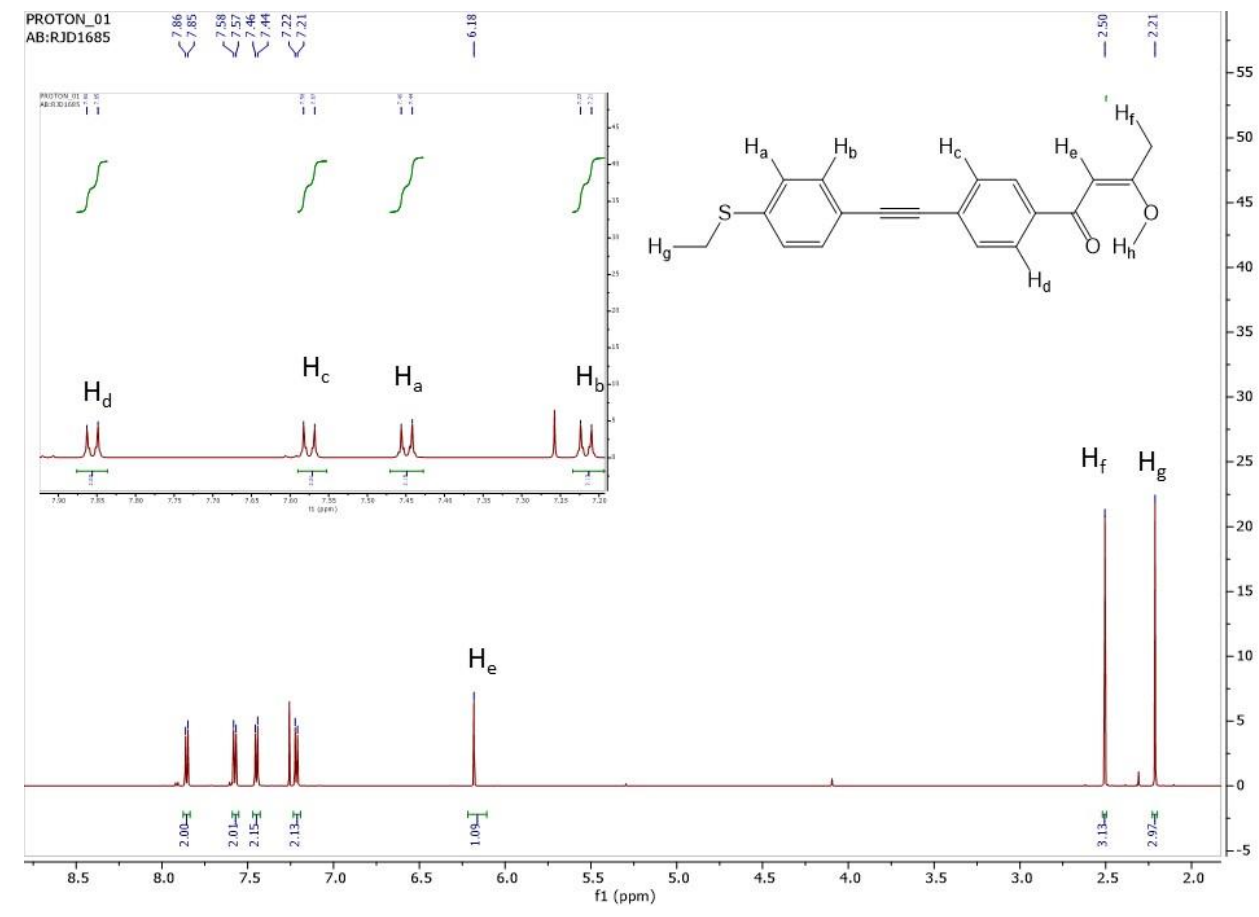

Figure S $13{ }^{1} \mathrm{H}$ NMR of 5 recorded in $\mathrm{CDCl}_{3}$

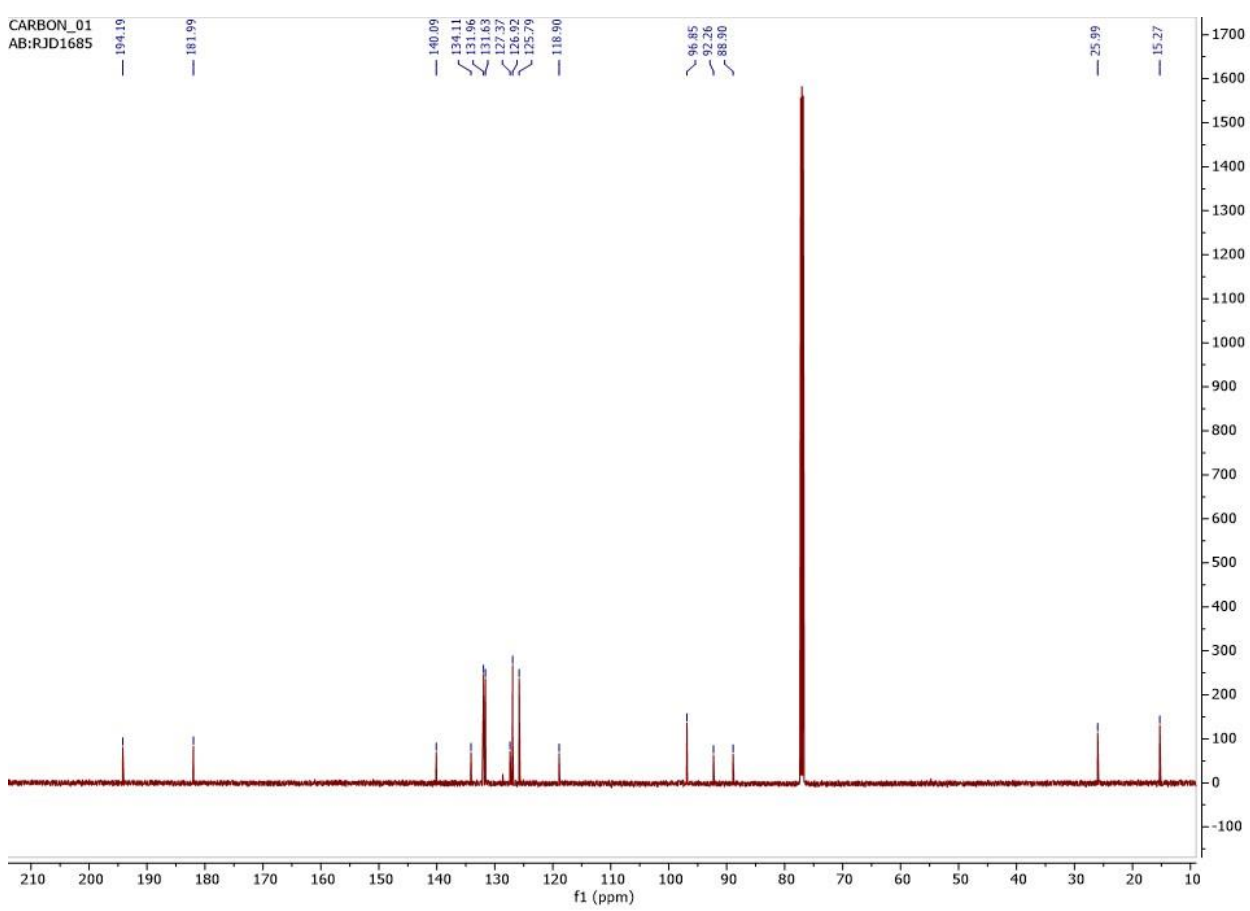

Figure $S 14{ }^{13} \mathrm{C} N \mathrm{NM}$ of 5 recorded in $\mathrm{CDCl}_{3}$ 


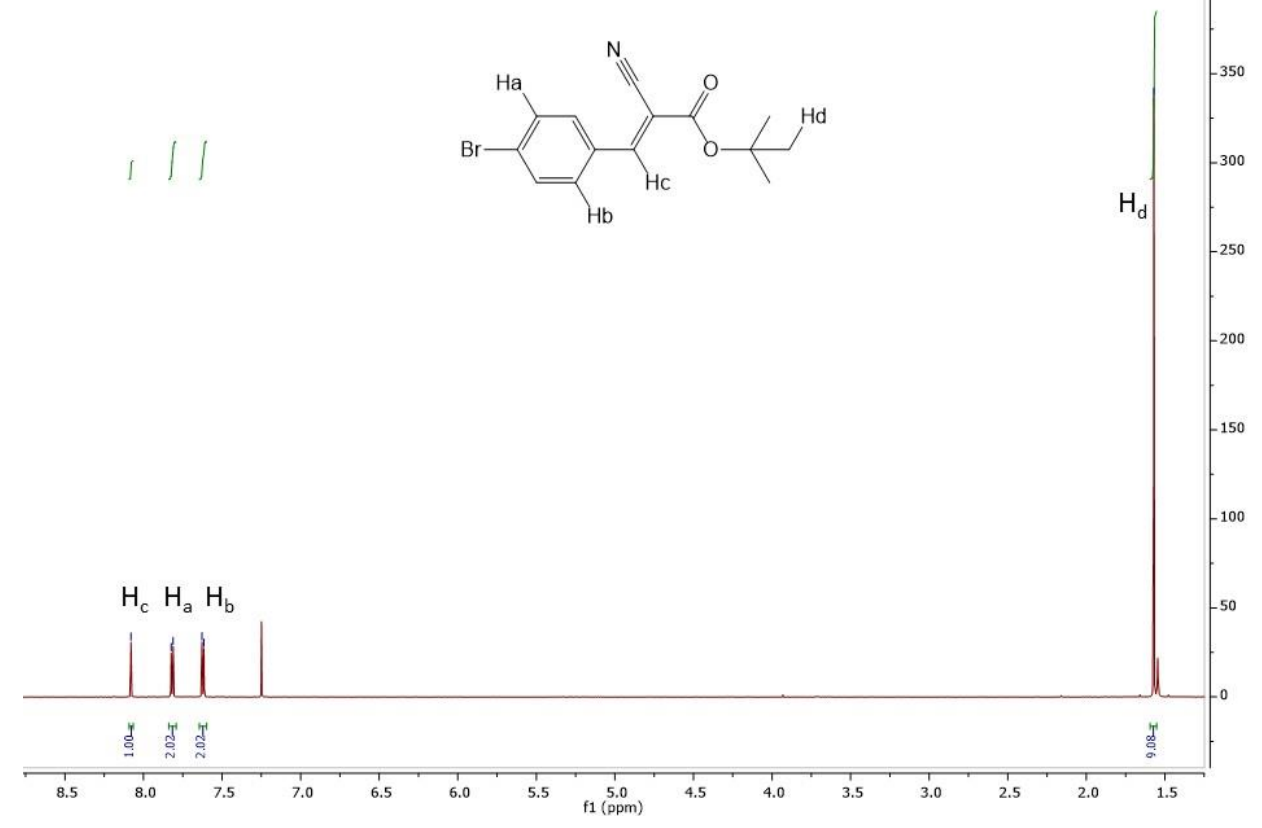

Figure $\mathrm{S}_{15}{ }^{1} \mathrm{H} \mathrm{NMR}$ of c recorded in $\mathrm{CDCl}_{3}$.

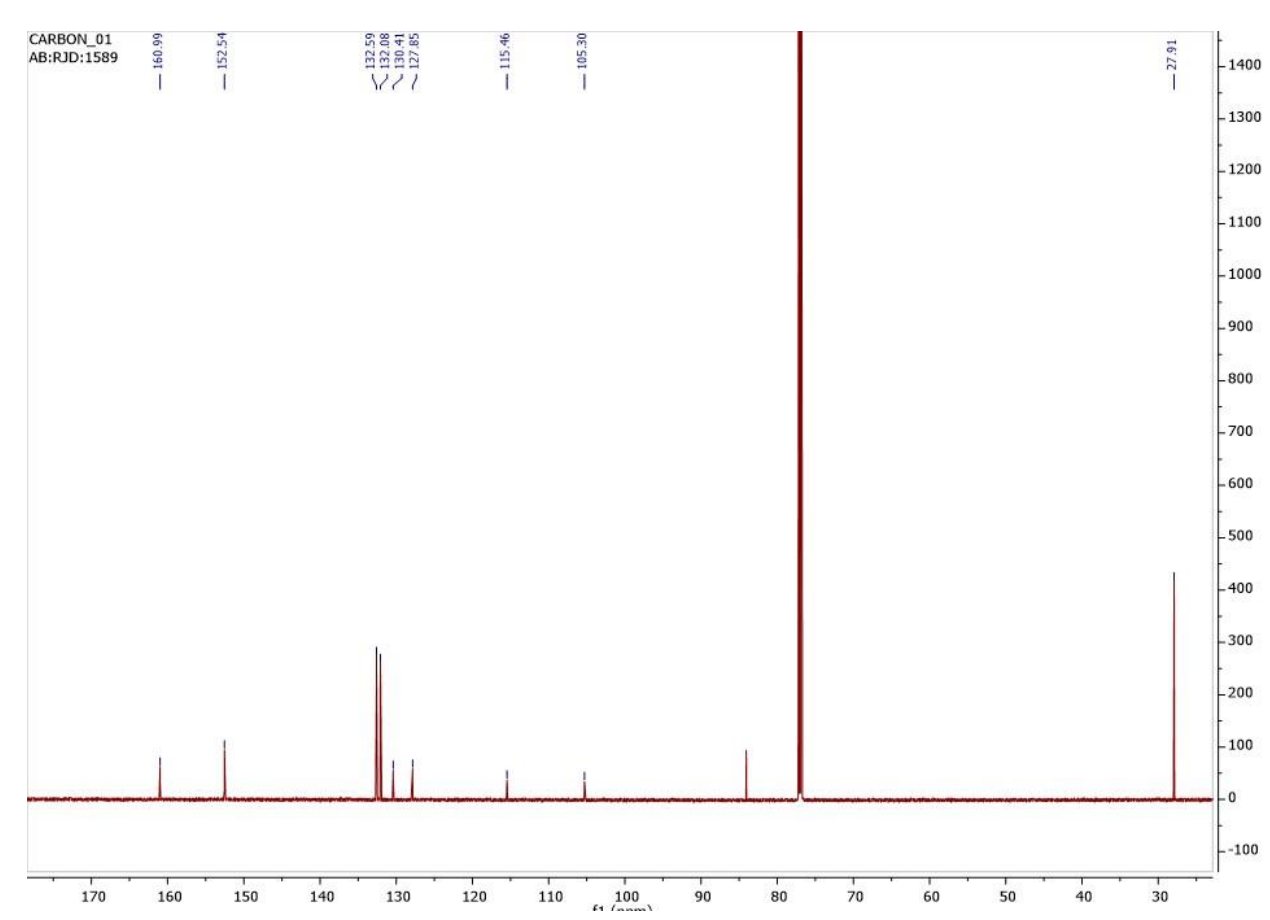

Figure $S 16^{13} \mathrm{C} \mathrm{NMR}$ of c recorded in $\mathrm{CDCl}_{3}$. 


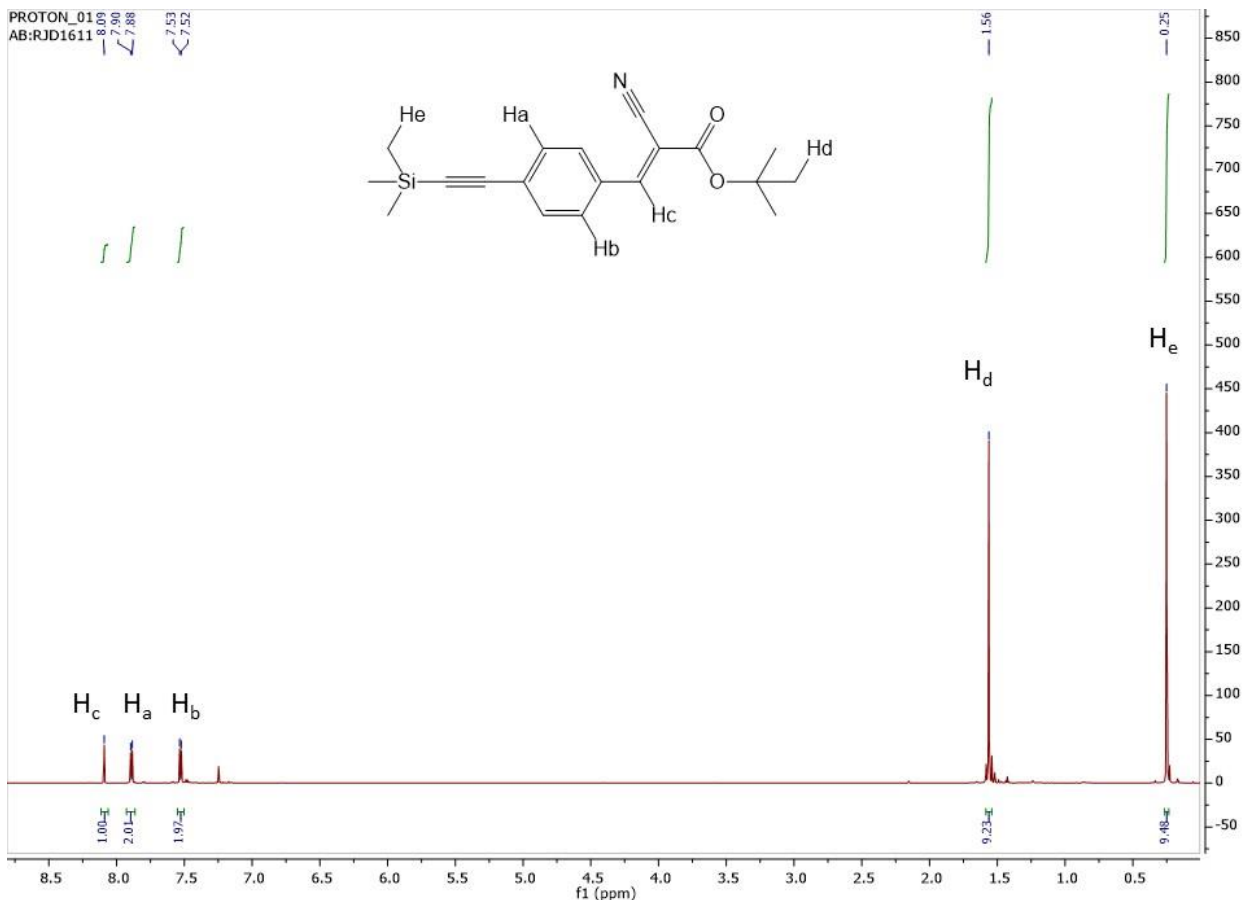

Figure $\mathrm{S}_{17}^{1} \mathrm{H} \mathrm{NMR}$ of d recorded in $\mathrm{CDCl}_{3}$.

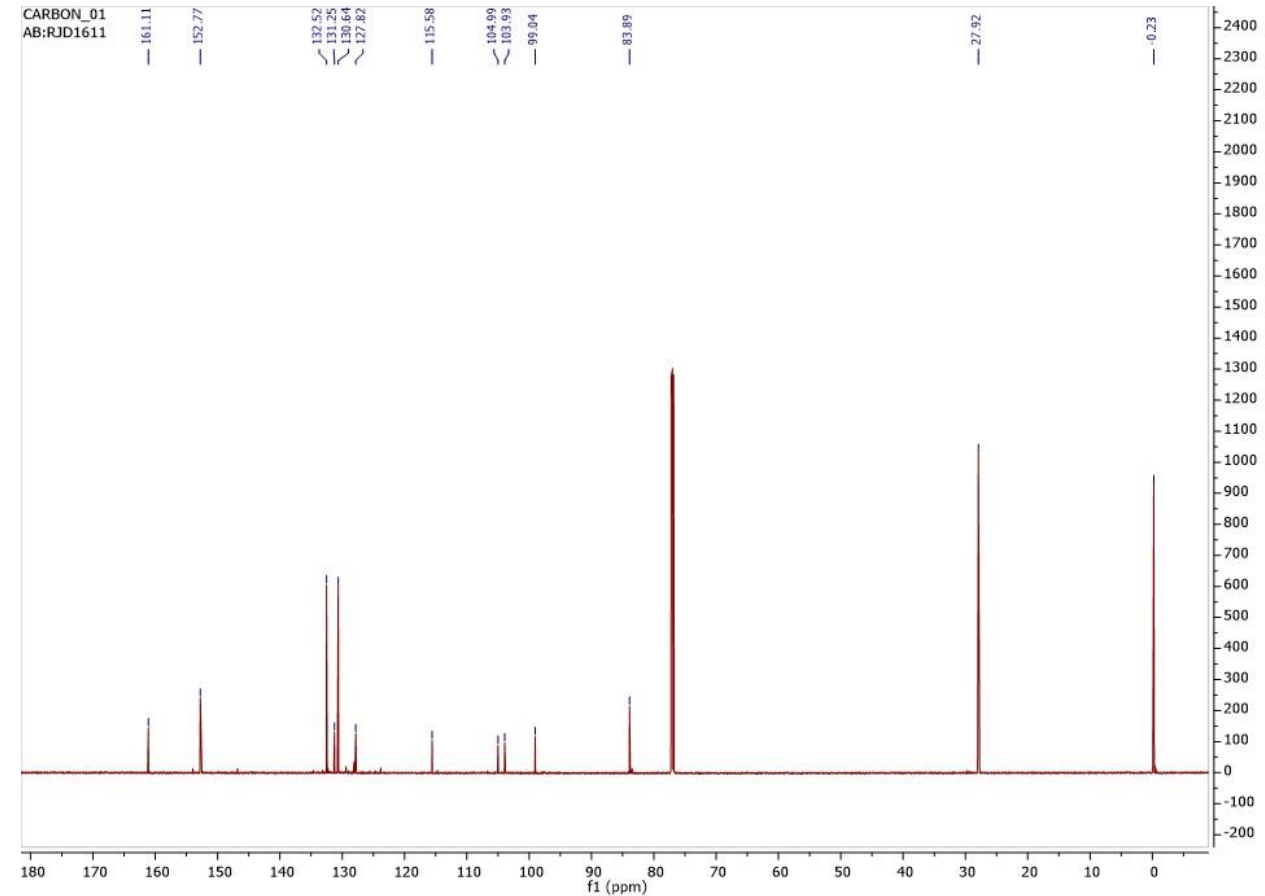

Figure $S 18{ }^{13} \mathrm{C} \mathrm{NMR}$ of d recorded in $\mathrm{CDCl}_{3}$. 


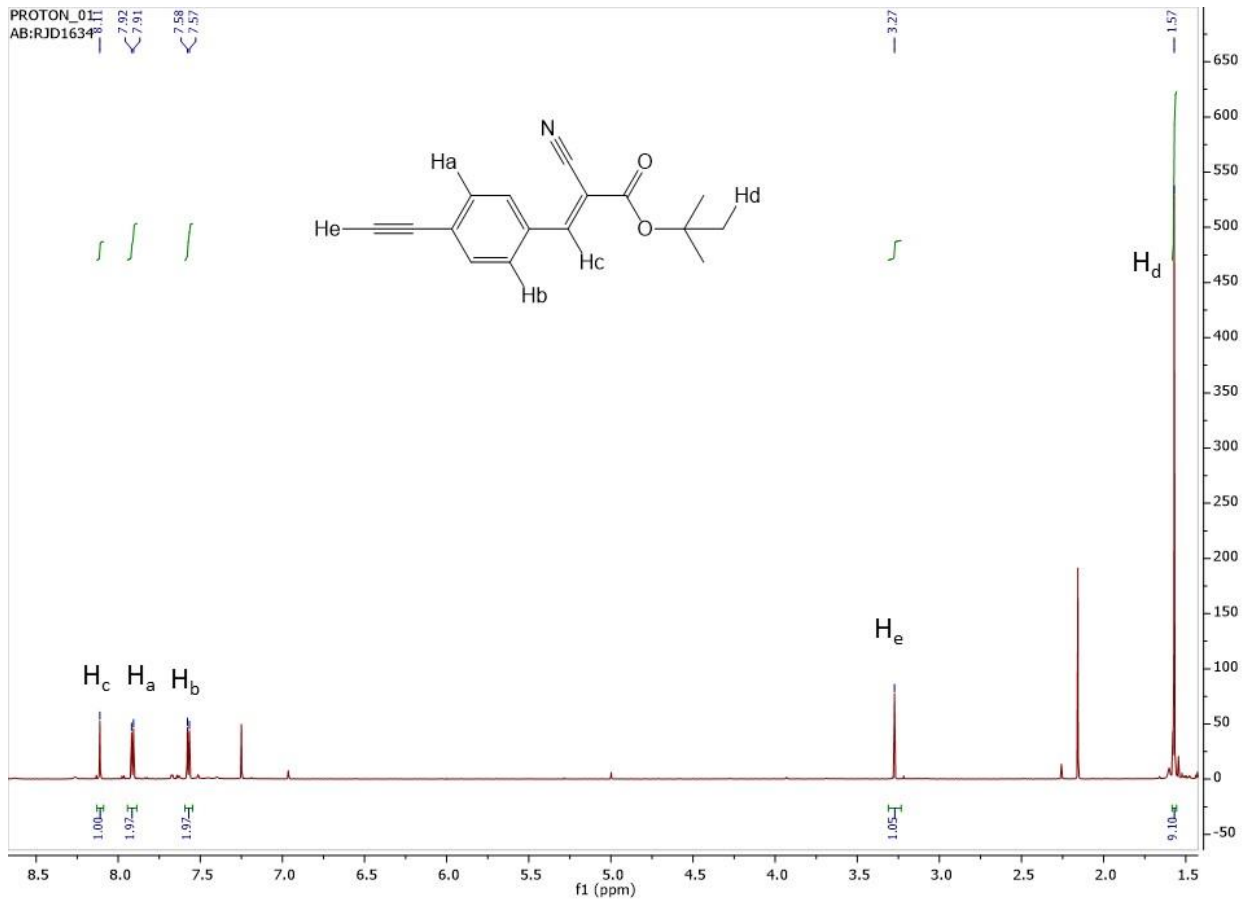

Figure $S 19^{1} \mathrm{H} N \mathrm{NMR}$ of e recorded in $\mathrm{CDCl}_{3}$.

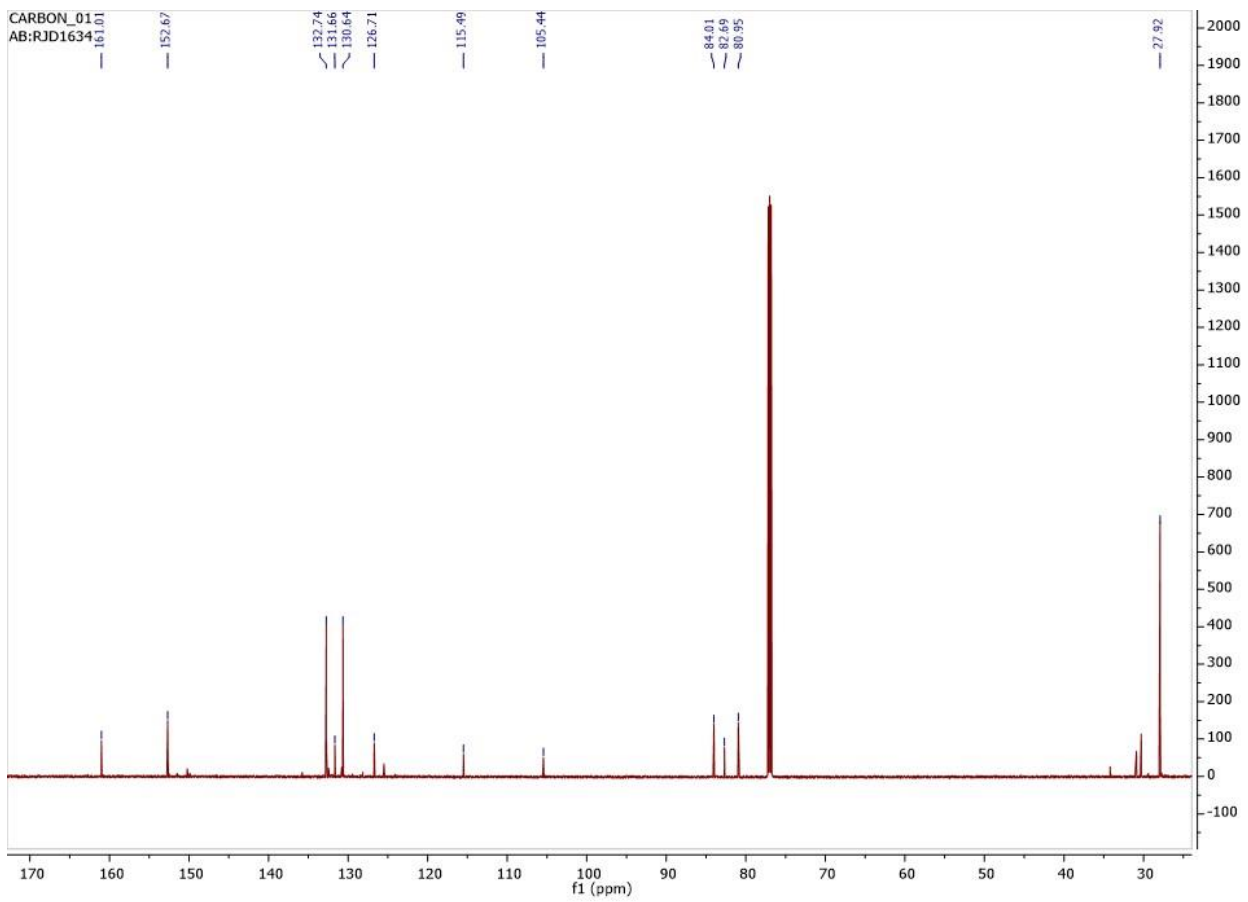

Figure $S 20{ }^{13} \mathrm{C} N \mathrm{NM}$ of e recorded in $\mathrm{CDCl}_{3}$. 


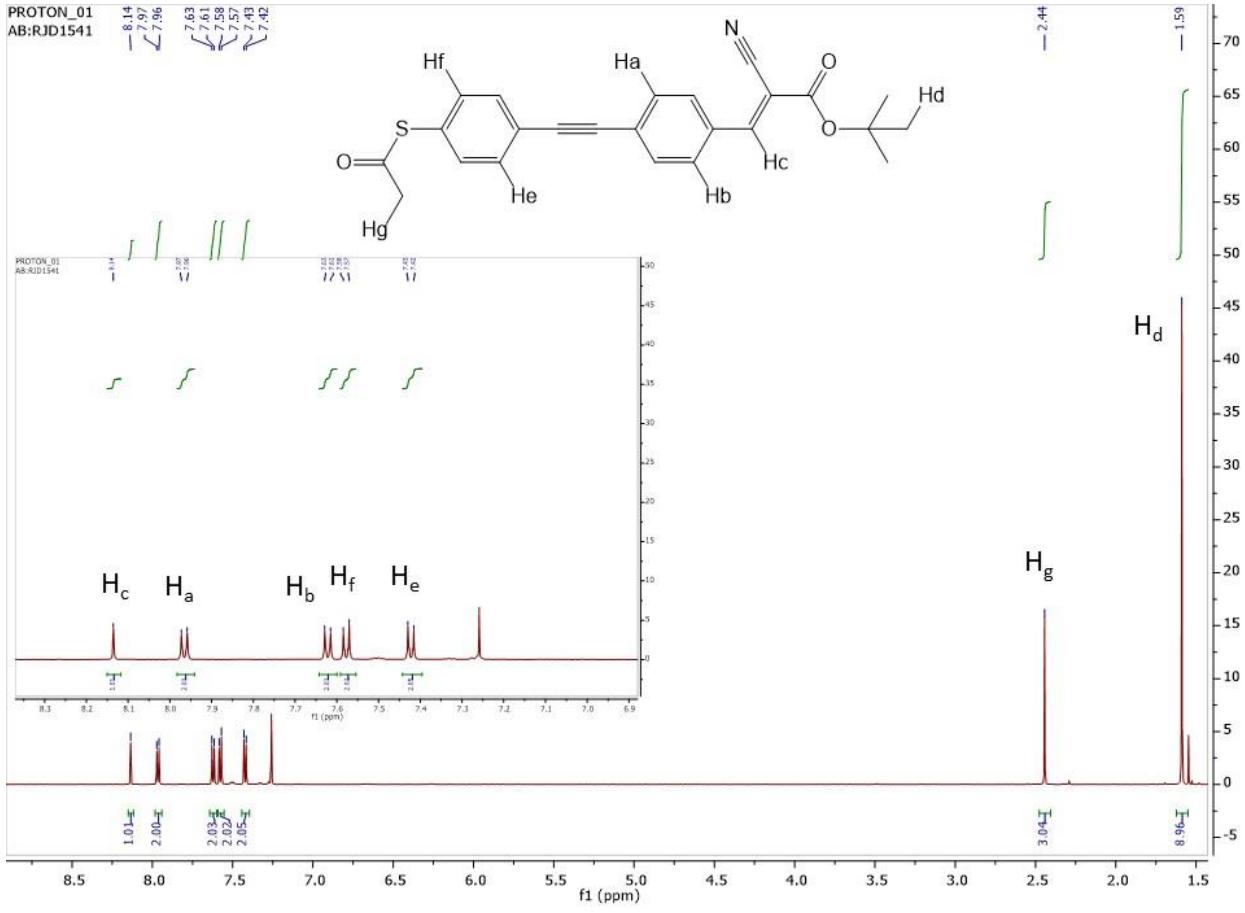

Figure $S 21{ }^{1} \mathrm{H} N M R$ off recorded in $\mathrm{CDCl}_{3}$.

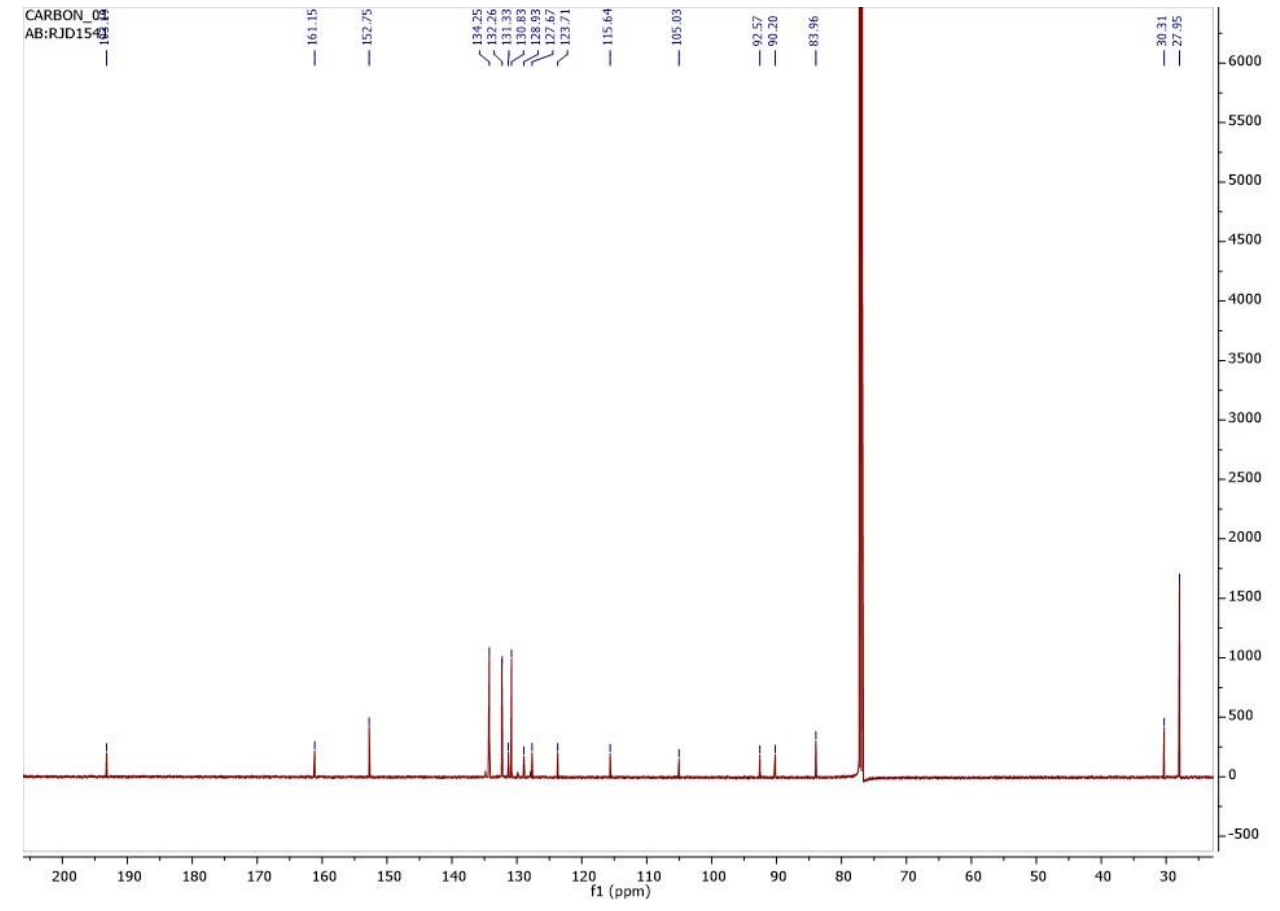

Figure $\mathrm{S} 22^{13} \mathrm{C}$ NMR off recorded in $\mathrm{CDCl}_{3}$. 


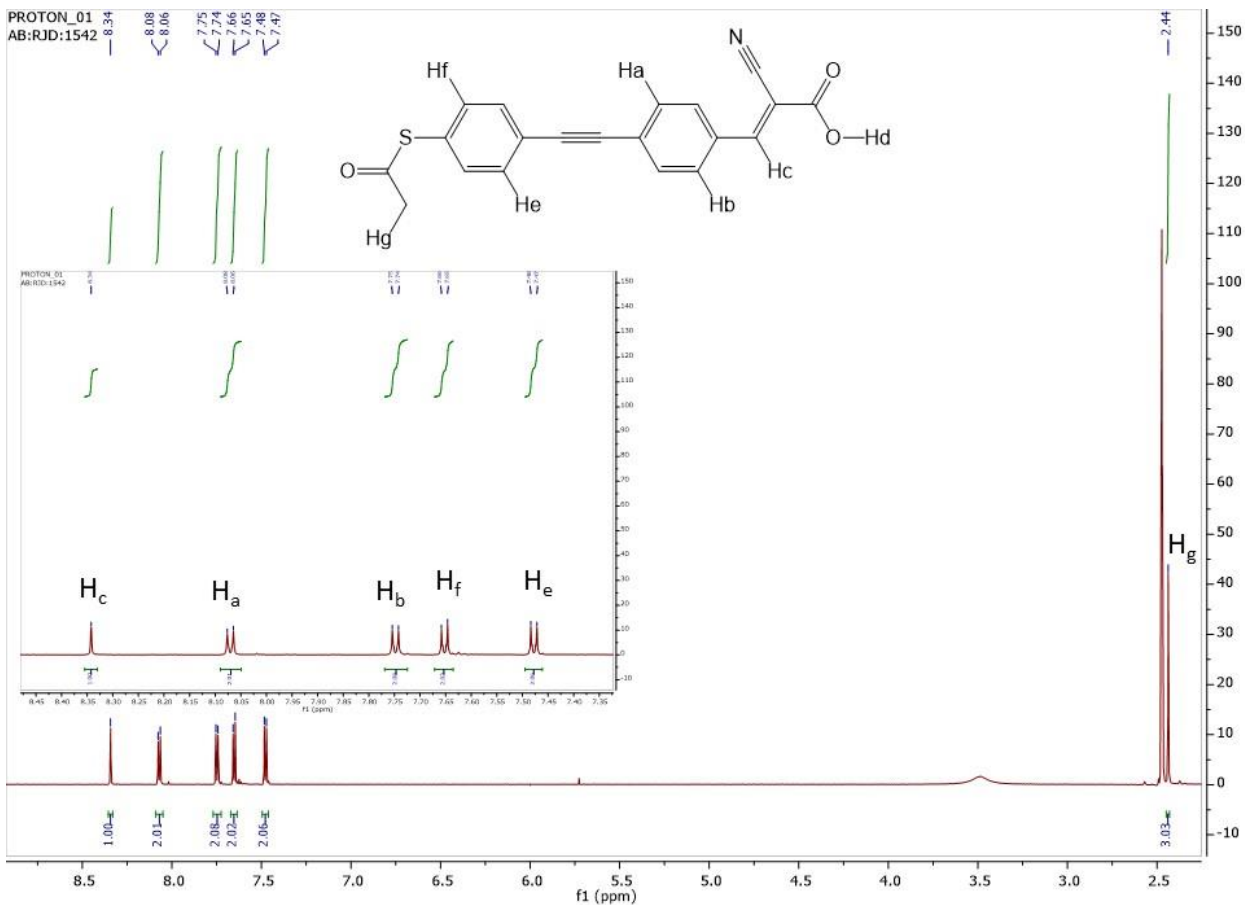

Figure S $2{ }^{1}{ }^{H}$ NMR of 7-acrylate recorded in DMSO-d6.

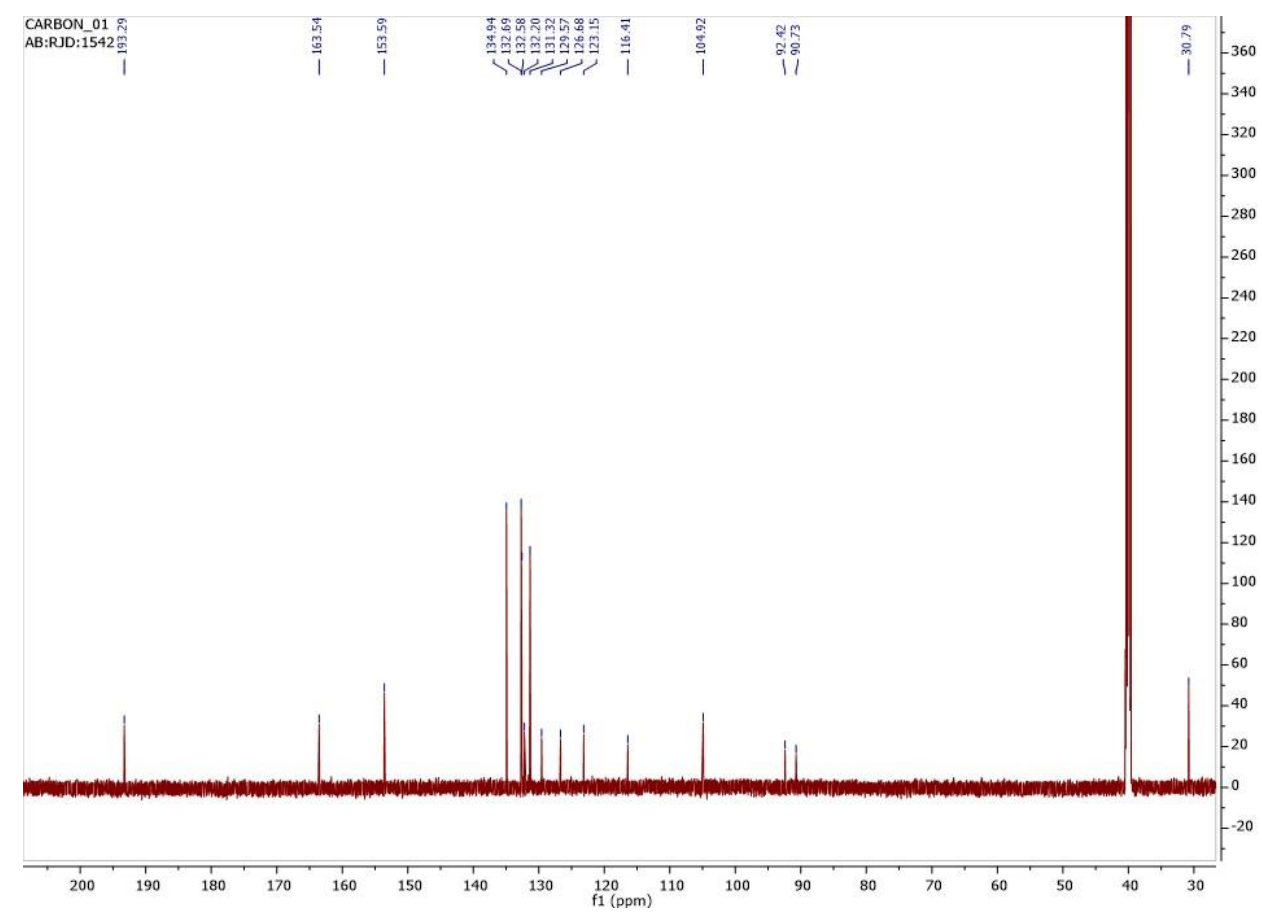

Figure S $24{ }^{13} \mathrm{C} N M R$ of 7-acrylate recorded in DMSO-d . $_{\text {. }}$ 


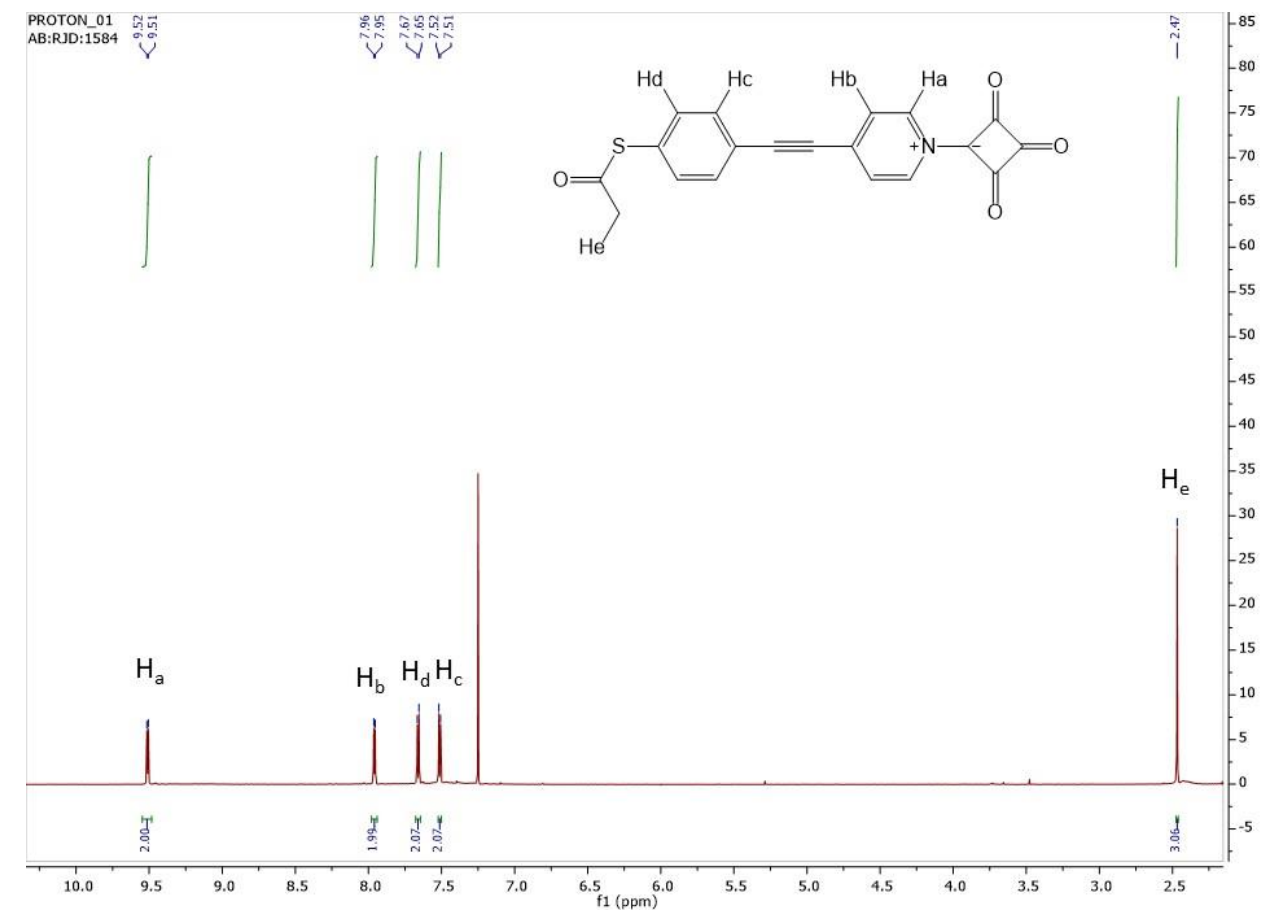

Figure S $25^{1} \mathrm{H} N M R$ of 8 -squarate recorded in $\mathrm{CDCl}_{3}$.

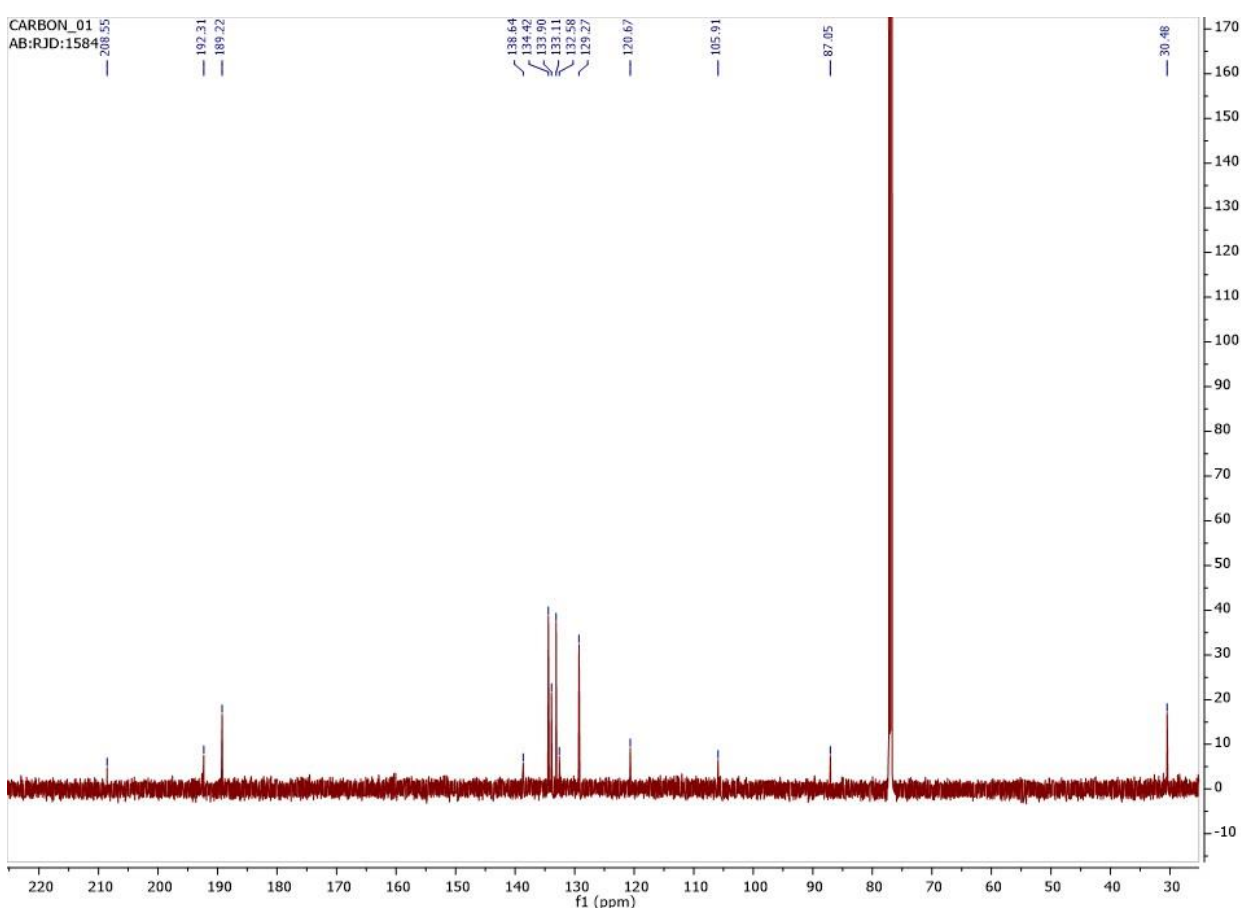

Figure $S 26{ }^{13} \mathrm{C} N M R$ of 8 -squarate recorded in $\mathrm{CDCl}_{3}$. 


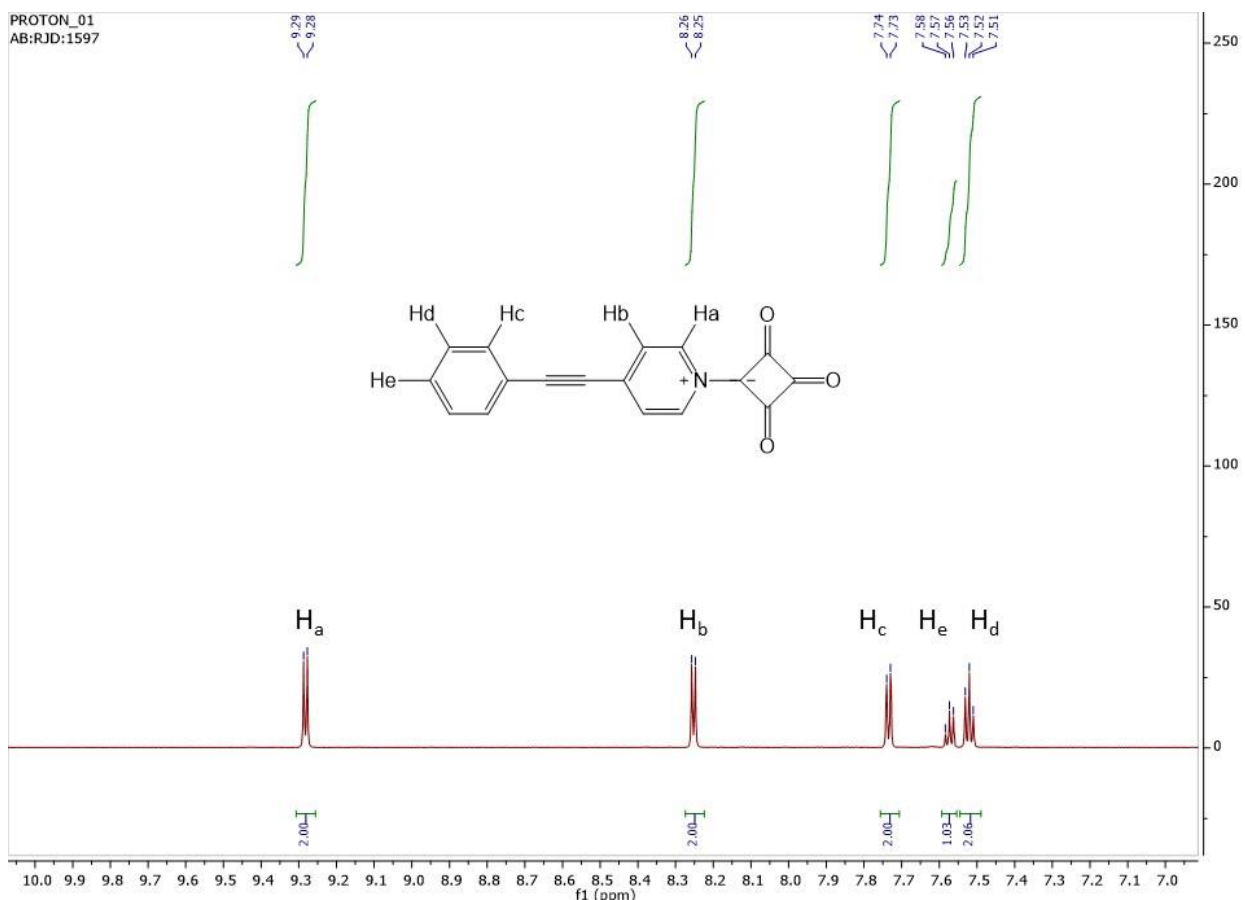

Figure S $27^{1} \mathrm{H}$ NMR of 9 recorded in DMSO-d

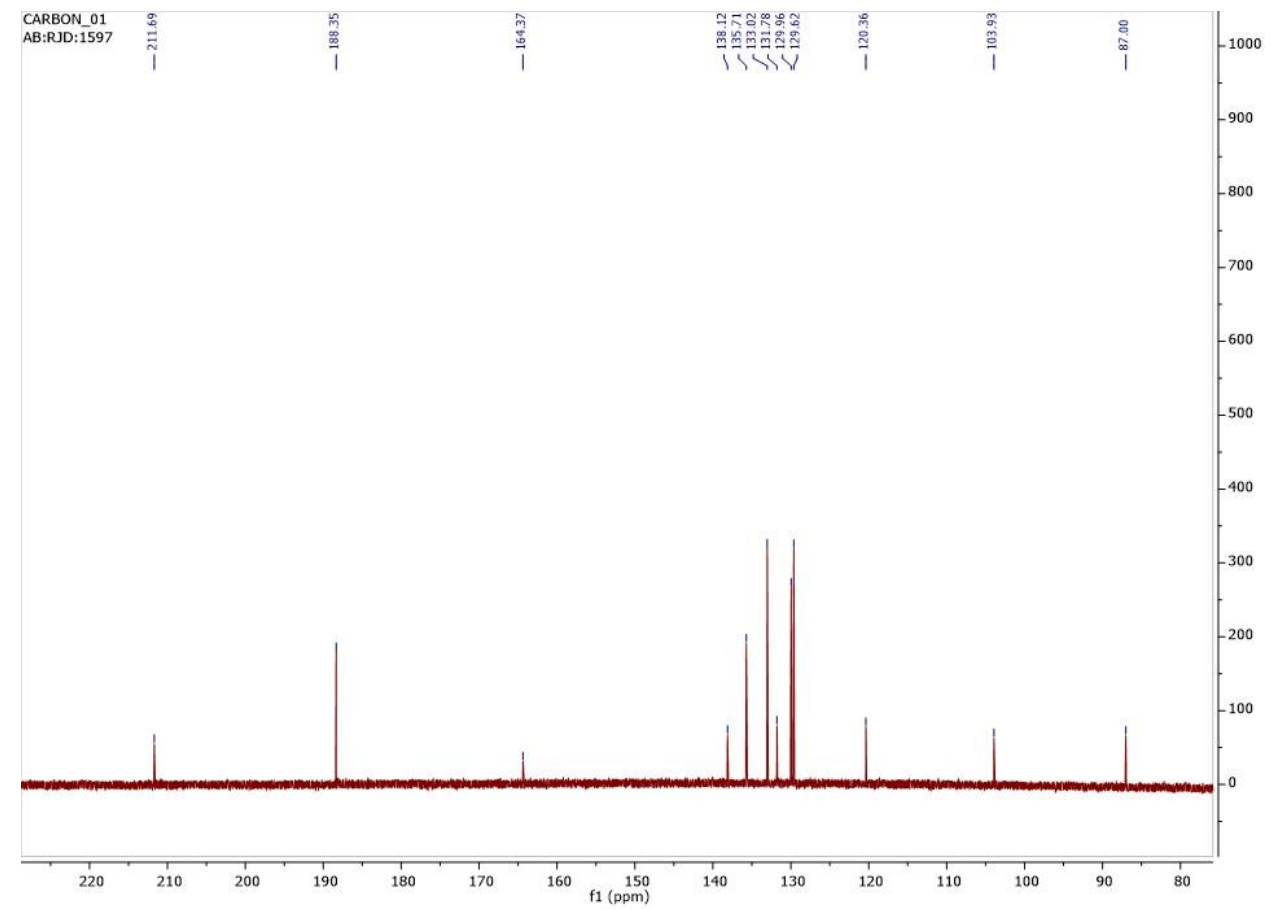

Figure S $28{ }^{13}$ C NMR of 9 recorded in DMSO-d. 


\section{STM Break-Junction details}

Single-molecule break-junction experiments were carried out using a modified STM (Keysight Technologies 5500 SPM). The system is equipped with a custom-built, 4-channel current amplifier based on the design originally reported by Meszaros et al. ${ }^{7}$ The signal from the 4 independent channels is recorded through a NI-9215 USB c-DAQ, with a sampling speed of $10 \mathrm{kHz}$. Tip positioning in the three axes is controlled by the STM electronics.

Before each experiment, a gold on glass substrate (Arrandee $\mathrm{GmbH}$ ) was rinsed with acetone and then gently flame-annealed using a butane torch while placed on top of a silicon wafer to avoid bending of the glass. The substrate was then mounted into a liquid STM cell, and a few drops of $1 \mathrm{mM}$ molecular solution were added. An STM tip was cut from a spool of $99.99+\%$ gold wire (Goodfellow, UK). All experiments were performed at a bias voltage of 100 or $200 \mathrm{mV}$.

The STM tip was repeatedly driven a few angstroms into the substrate and subsequently withdrawn several nanometres, all at constant bias voltage, retraction speed, and while maintaining a fixed $x-y$ position. The current signal (as a raw voltage) was continuously recorded during approach and withdrawal of the tip.

Raw data was converted into conductance-distance traces using a Python script (v.2.7, Python Software Foundation, https://www.python.org). Only data corresponding to the withdrawal portion of the experiment was used for analysis, and the approach data is discarded. Several thousand traces were collected for each molecule and presented without selection in logarithmically binned conductance histograms.

Figure S 29 shows gold-molecule-gold conductance histograms for all eight tolane wires. Clear molecular peaks are presented in panels $B$ and $G$, which together form group $A$ of the main text. Panels $D, F$, and $H$ show the results for group $B$ of the main text, with a mixed success rate for ITO selectivity. Finally, panels $A, C$, and $E$ show the results for the three thiol-functionalised wires that we selected for further analysis.

From Figure $S 29$ it is clear that different contacting groups give different propensities for junction formation in gold-molecule-gold break junctions. 6-carboxylate and 1 only differ in one anchoring group, namely thiol versus DMBT. The DMBT surface binding group has been shown as a very effective anchor for gold break junctions (ref. 20 of main manuscript) and indeed here this is demonstrated by a strong histogram peak for 1 (Panel B of Figure $S$ 29) compared to a very weak peak for 6-carboxylate (Panel A). 7-acrylate (Panel E) and $\mathbf{5}$ $($ Panel $\mathrm{H}$ ) both give distinguishable histogram peaks but they are clearly not as effective at forming defined junctions as $\mathbf{1}$ (Panel B) or $\mathbf{2}$ (Panel G). Counterintuitively, 2 (Panel G) forms 
more distinctive histograms than 4 (Panel F). These collected results show a complex pattern of junction formation propensities for these gold-molecule-gold junctions depending on molecular structure, which could arise from junction formation energetics or dynamics. On the other hand, $\mathbf{3}$ and 8-squarate show no signs of junction formation in gold-molecule-gold break junctions. This gives the most important result of this screening which is that the two compounds with a squarate anchoring group give no junction formation propensity with gold electrodes.
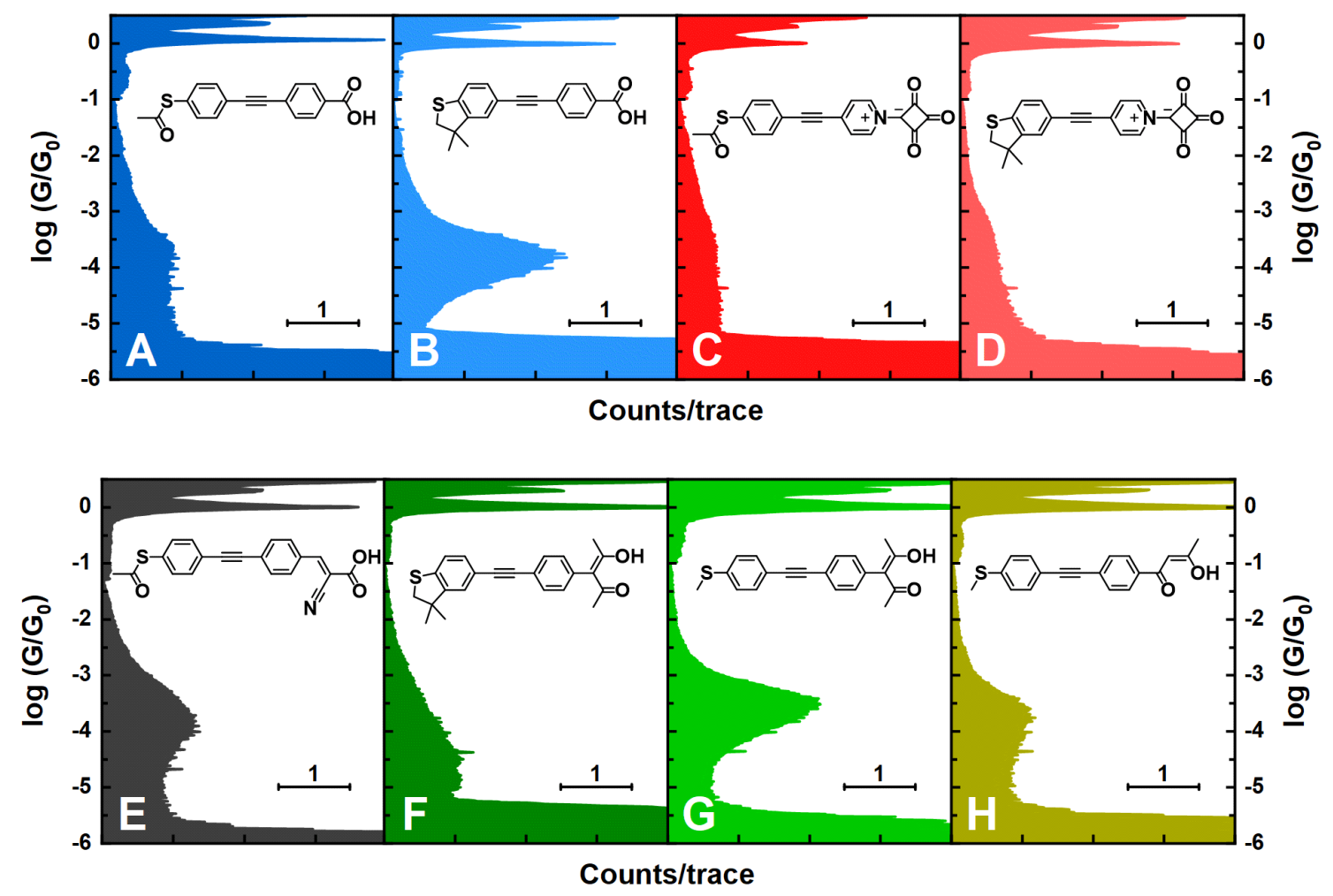

Figure S 29 Single-molecule conductance histograms show the presence or absence of gold-molecule-gold junctions. Insets show molecular structures of each wire. Histograms in $C, D$, and $F$ do not show a molecular feature. Junction formation probability varies between histograms that show a molecular feature; High formation probability in $B$ and $G$, and lower formation probability in $A, E$, and $H$. 


\section{Absorption spectroscopy of wires 6-carboxylate, 7-acrylate, and}

\section{8-squarate}

UV-VIS absorption measurements were carried out on a Jenway 7305 spectrophotometer in appropriate solvents, 6-carboxylate and 7-acrylate in the same solvent as their STM-BJ conductance measurements, see Figure S 30. These electronic spectra show that the main absorption bands shift with structure. Since the molecules have similar conductance values it suggests that contact chemistry is having a large influence on the observed conductances.

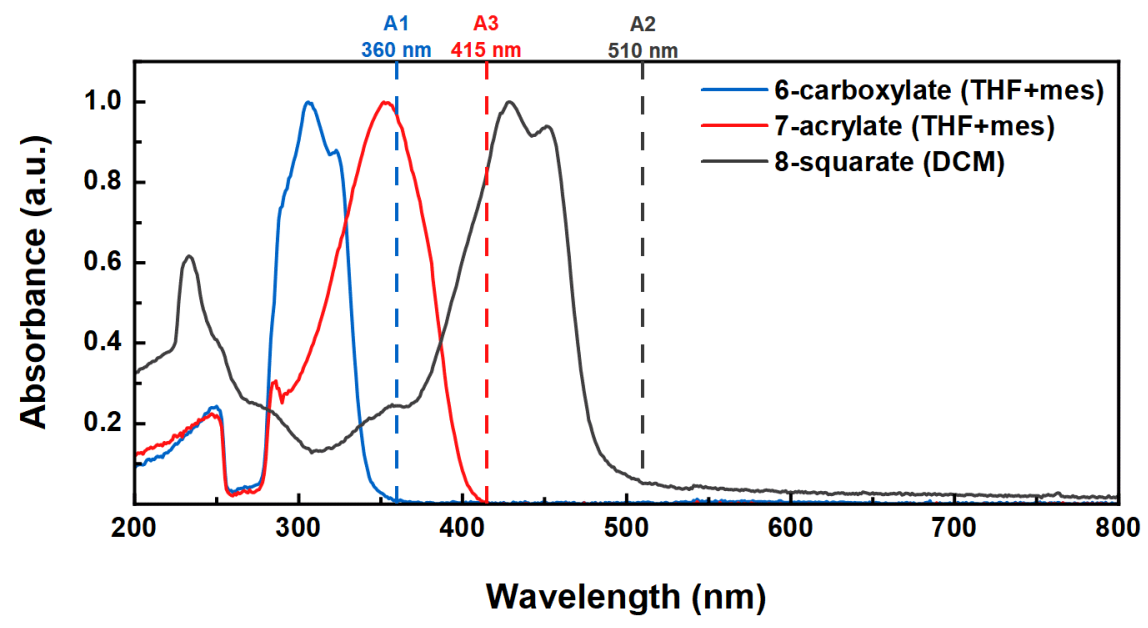

Figure S 30 Optical band-gap estimation from UV-VIS absorption spectra shows that 8-squarate has the smallest gap, followed by 7-acrylate and then 6-carboxylate. Absorption profiles for wires 6-carboxylate (blue), 7-acrylate (gray), and 8-squarate (red). The dotted lines show estimated shoulders for the HOMO-LUMO gaps of each wire. 


\section{Surface characterisation experiments (XPS, QCM, AFM)}

X-ray photoelectron spectroscopy (XPS) was used to gain insight into the binding interactions between these specific anchoring groups (carboxylic acid, cyanoacrylic acid and pyridinium squarate) and the different substrates used (ITO and gold). For this purpose adsorbed monolayers of these compounds were formed on both substrates from $10^{-4} \mathrm{M}$ solutions in THF:chloroform (1:4), prior to transferring to the UHV XPS chamber for analysis. A Kratos AXIS Ultra DLD spectrometer equipped with a monochromatic Al K $\alpha$ X-ray source $(1486.6 \mathrm{eV})$ with a pass energy of $20 \mathrm{eV}$ and a photoelectron take-off angle of $90^{\circ}$ with respect to the sample plane was used. The $\mathrm{C} 1 \mathrm{~s}$ peak at $284.6 \mathrm{eV}$ was used for calibration of energies. The middle column of Figure $S 31$ shows the XPS data for the S2p region for the powder sample of 6-carboxylate, 7-acrylate, and 8-squarate. All these powder samples display two peaks with an area ratio of $\sim 2: 1$ which are assigned to $\left(2 p_{3 / 2}\right)$ and $\left(2 p_{1 / 2}\right)$, respectively, with the splitting arising from spin-orbit effects. The respective area ratios for the $2 p_{3 / 2}$ and $2 p_{1 / 2}$ peaks are $60.6 \%$ and $39.4 \%$ for 6 -carboxylate, $61.1 \%$ and $38.9 \%$ for 7 acrylate and $61.8 \%$ and $38.2 \%$ for 8-squarate. The peaks lie at 163.5 and $164.7 \mathrm{eV}$ for 6carboxylate, 163.4 and $164.5 \mathrm{eV}$ for 7-acrylate and 163.3 and $164.5 \mathrm{eV}$ for 8-squarate (peak separation of $\sim 1.2 \mathrm{eV}$ ). ${ }^{8-9}$

By contrast, the XPS data from SAM of 6-carboxylate, 7-acrylate, and 8-squarate on a gold substrate are more convoluted (Figure $S 31$, left column). For 6-carboxylate, the XPS data displays three peaks at 164.8, 163.6 and $162.2 \mathrm{eV}$ (Figure S 31, top left). The peak at $164.8 \mathrm{eV}$ and the corresponding stronger $2 \mathrm{p}_{3 / 2}$ peak at $163.6 \mathrm{eV}$ (1.2 eV higher in energy) appear at practically the same binding energy as those observed for the powder sample (164.7 and $163.5 \mathrm{eV}$ ), revealing that 6-carboxylate does interact with the gold substrate exclusively through the thiol end. The lower binding energy peak at $162.2 \mathrm{eV}$ is assigned to the $2 p_{3 / 2}$ peak of a sulfur atom in contact with the gold substrate. This $2 p_{3 / 2}$ peak would pair with a weaker $2 p_{1 / 2}$ peak, which would be expected to fall ca. $1.2 \mathrm{eV}$ higher in energy (i.e. at $163.4 \mathrm{eV}$ ). The observed signal is, therefore, the convolution of contributions from surfacebonded and free sulfur atoms. ${ }^{10-12}$ This leads us to conclude that in the case of SAMs of 6carboxylate, adsorbate molecules are adsorbed onto the gold substrate through either the thiol or the carboxylic group, without pronounced selectivity. The relative areas of the XPS peaks from sulfur not bonded and bonded to the gold substrate are 35:65 (Figure S31), implying that sulfur has a slight predominance to be bonded to gold in SAMs of 6carboxylate. 
Gold

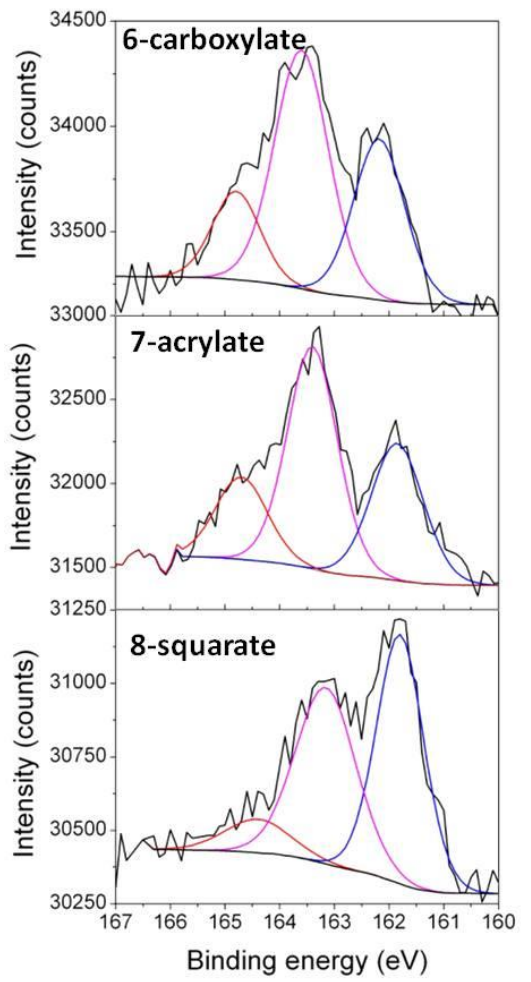

Powder

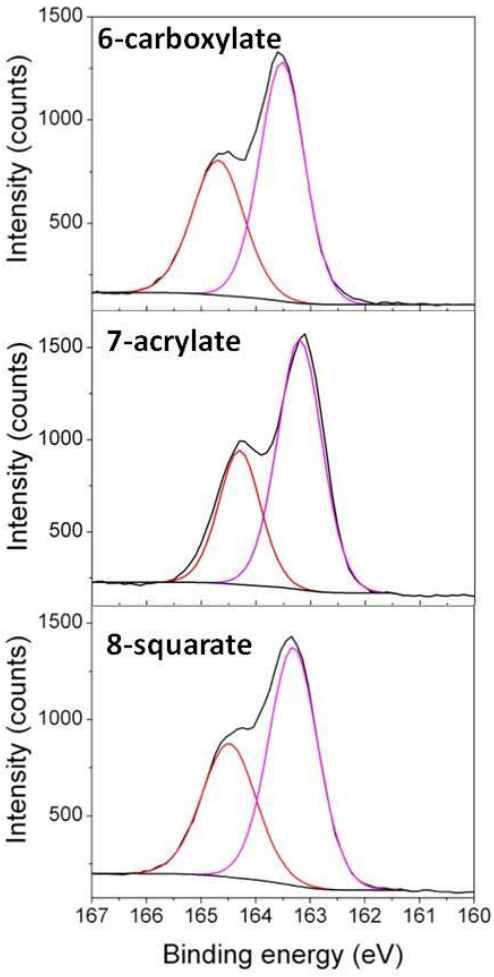

ITO

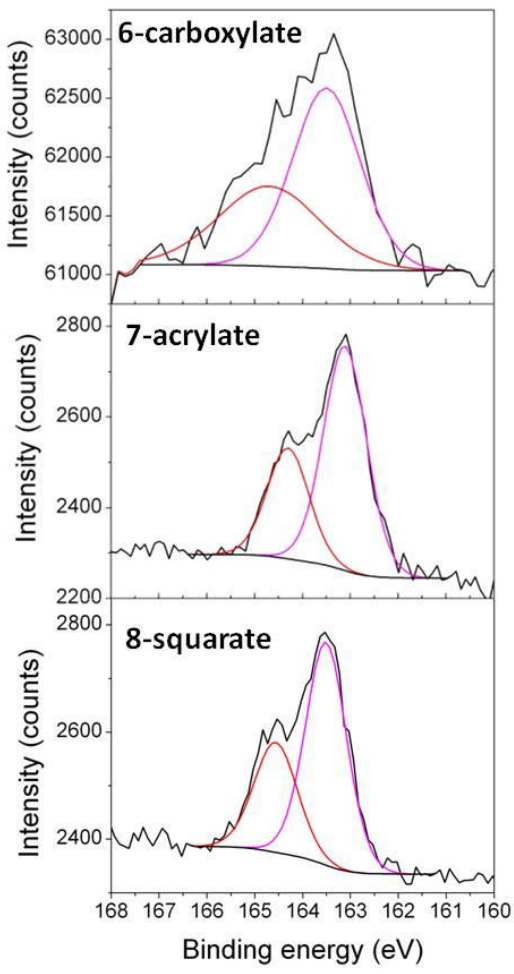

Figure S 31 XPS data recorded in the S2p region for 6-carboxylate, 7-acrylate, and 8squarate as powder (middle) as a SAM on gold (left) and as SAM on ITO (right).

Similar XPS results are obtained for 7-acrylate. The XPS data from SAM of 7-acrylate on gold shows three peaks at 164.7, 163.4 and $161.9 \mathrm{eV}$ (Figure S 31, middle left). The peak at $164.7 \mathrm{eV}$ and the part of the corresponding stronger $2 \mathrm{p}_{3 / 2}$ peak at $163.5 \mathrm{eV}$ are attributed to thiol moieties not bound to the gold substrate supporting the monolayer. On the other hand, the peak at $161.9 \mathrm{eV}$ is assigned to the $2 \mathrm{p}_{3 / 2}$ peak of a thiol sulfur atom bound to the gold substrate, and this is paired with a corresponding weaker $2 p_{1 / 2}$ peak expected to fall ca. 1.2 $\mathrm{eV}$ higher in energy (i.e. at $163.1 \mathrm{eV}$ ). Thus, the SAM of 7-acrylate shows similar XPS behaviour to that of 6-carboxylate, with the molecules either contacting the gold substrate through the thiol or the cyanoacrylic end. However, when comparing 7-acrylate with 6carboxylate, the former shows higher ratios of free to surface-bonded sulfur with area ratios of 52:48 (Figure S 31, middle left).

Finally, XPS data for a SAM of 8-squarate on gold shows two intense peaks at 163.2 and $161.8 \mathrm{eV}$ (Figure S 31, bottom left) and a weak peak at $164.4 \mathrm{eV}$. The strong peak at 161.8 $\mathrm{eV}$ is assigned to the $2 \mathrm{p}_{3 / 2}$ feature of sulfur bonded as thiol to the gold surface. This would pair with a weaker $2 \mathrm{p}_{1 / 2}$ peak, that is expected to fall ca. $1.2 \mathrm{eV}$ higher in energy, i.e. at 163.0 $\mathrm{eV}$ which aligns well with the peak observed at $163.2 \mathrm{eV}$. The very weak peak at $164.4 \mathrm{eV}$ is 
at the energy expected for the $2 p_{1 / 2}$ resonance of sulfur not bonded to the gold surface. We conclude that molecules in a SAM of 8-squarate interact with the gold substrate preferably via the thiol group, with very little surface binding through the pyridinium squarate group. The relative areas of XPS signals arising from thiols not bonded and bonded to the gold substrate, 16:84 (Figure S 31, bottom left) support this conclusion. This result was further confirmed using a quartz crystal microbalance (QCM). A QCM resonator was incubated in a $10^{-4} \mathrm{M}$ solution of $\mathbf{9}$ (a tolane with only a pyridinium squarate terminus) in THF:chloroform $(1: 4)$ and the frequency was monitored with time. After 48 hours of incubation, no variation in the frequency with respect to the bare QCM resonator was observed, demonstrating no adsorption of pyridinium squarate-capped molecules onto the gold substrate.

However, when an ITO substrate was used to support the SA monolayers of 6-carboxylate, 7-acrylate, and 8-squarate, the XPS data show only two peaks at 164.7 and $163.5 \mathrm{eV}$ for 6carboxylate (Figure S 31, top right), at 164.5 and $163.3 \mathrm{eV}$ for 7-acrylate (Figure S 31, middle right) and at 164.6 and $163.5 \mathrm{eV}$ for 8-squarate (Figure S 31, bottom right). All these peaks appear at practically the same position to those observed for the respective powders indicating that when an ITO substrate is used to support the monolayer the interaction of the molecules of 6-carboxylate, 7-acrylate, and 8-squarate is through the carboxylic acid, cyanoacrylic acid and pyridinium squarate, respectively, with the thiol group facing away from the surface and not in contact with the ITO. Therefore, these results demonstrate the specific affinity of the carboxylic acid, cyanoacrylic acid and pyridinium squarate towards an ITO substrate versus the thiol group.

Next, the N1s region was analysed at high resolution, to obtain more information about the interaction of the new pyridinium squarate adsorbate group with an ITO substrate. The XPS data for the powder shows an intense peak at $401.6 \mathrm{eV}$ attributed to the positively charged nitrogen $\left(\mathrm{N}^{+}\right),{ }^{13}$ and a less intense peak at $398.6 \mathrm{eV}$ associated to the radical cation formed during X-ray excitation in the analysis chamber of the XPS instrument (Figure $\mathbf{S}$ 32, top panel). ${ }^{14}$ For a SAM of 8-squarate on ITO two peaks appear at the same binding energies to these observed for the powder; indicating the absence of an interaction between the $\mathrm{N}$ atom of the pyridinium squarate group and the substrate (Figure $\mathbf{S} 32$, bottom panel). Thus, the interaction of the pyridinium squarate group with the ITO has to occur through the $\mathrm{O}$ atoms. It is noteworthy to indicate here that the high-resolution O1s region was not analysed since an ITO substrate is expected to have multifarious oxygen species which would mask any potential peaks attributed to the pyridinium squarate. 


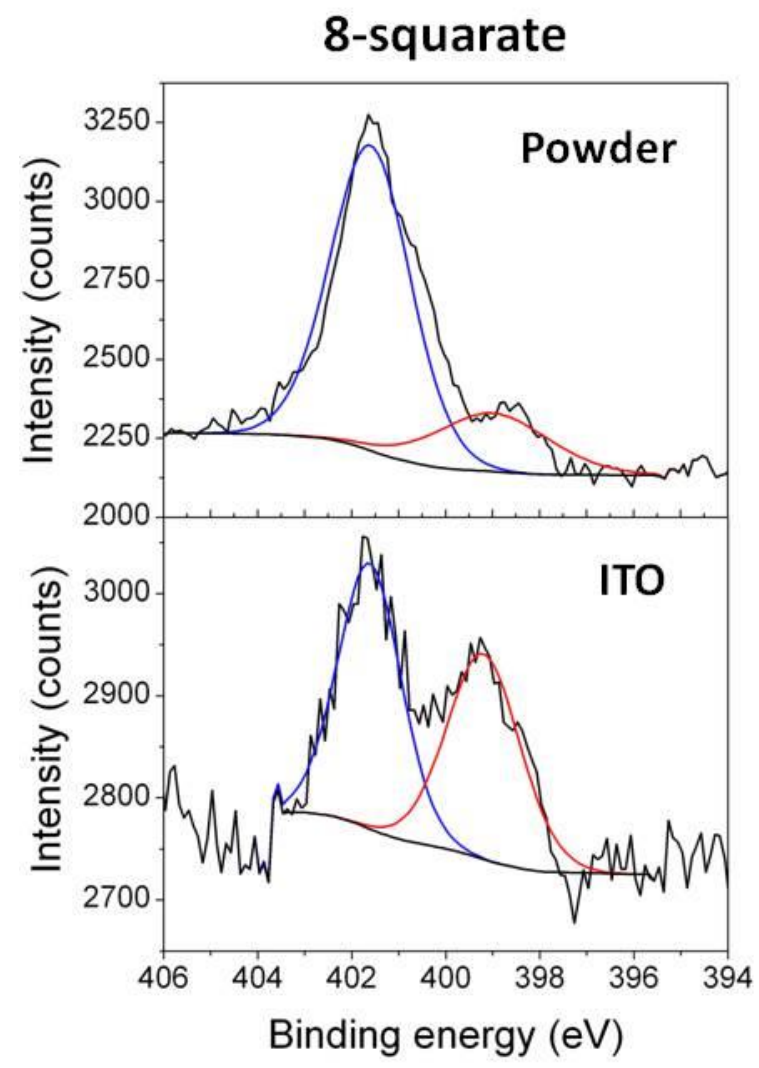

Figure S 32 XPS data recorded in the N1s region for 8-squarate as powder and as SAM on ITO.

For the nanolithographic indentation of a molecular SAM on ITO, the substrate was mounted into a commercial AFM (Keysight Technologies 5500 SPM) equipped with a contact mode nose cone and a silicon cantilever. The laser was aligned onto the centre of the photodiode before approaching. Once in contact, a surface image was recorded in contact mode using a force setpoint of $\sim 5 \mathrm{nN}$, followed by reducing the scan area to record a second smaller image in the centre of the first. This second image was recorded at a force of $\sim 20 \mathrm{nN}$, after which the first scan was repeated at $\sim 5 \mathrm{nN}$.

AFM contact mode images of an ITO substrate with an adsorbed layer of 6-carboxylate present (top) are contrasted with a bare ITO substrate (bottom) in Figure S 33. In each case, the left panel shows a scan of the ITO substrate prior to applying a high contact force. The inset in the centre shows a magnified (zoomed-in) area where the large contact force was applied. The right panel shows the same scan area as the left panel after zooming back out after application of the high contact forces. The substrate that had a monolayer (top) clearly shows that it has been damaged or deformed by the high contact force, whereas the bare substrate (bottom) shows no signs of damage. 
6-carboxylate self-assembled on ITO surface

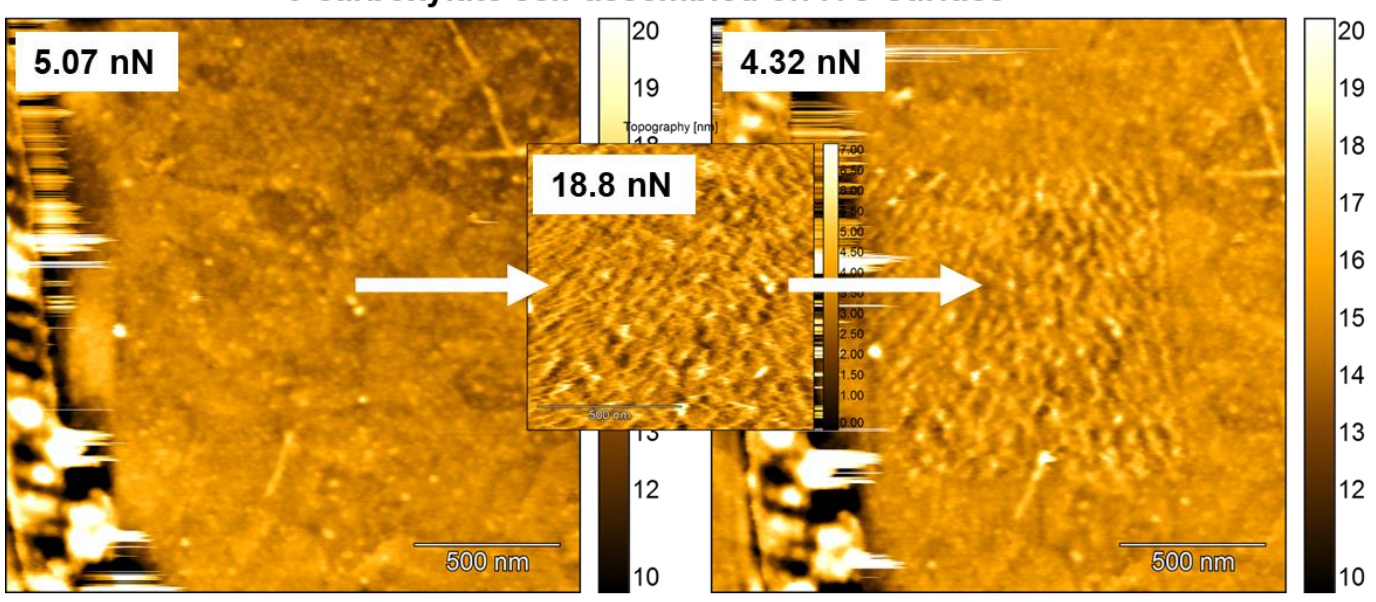

Clean ITO surface

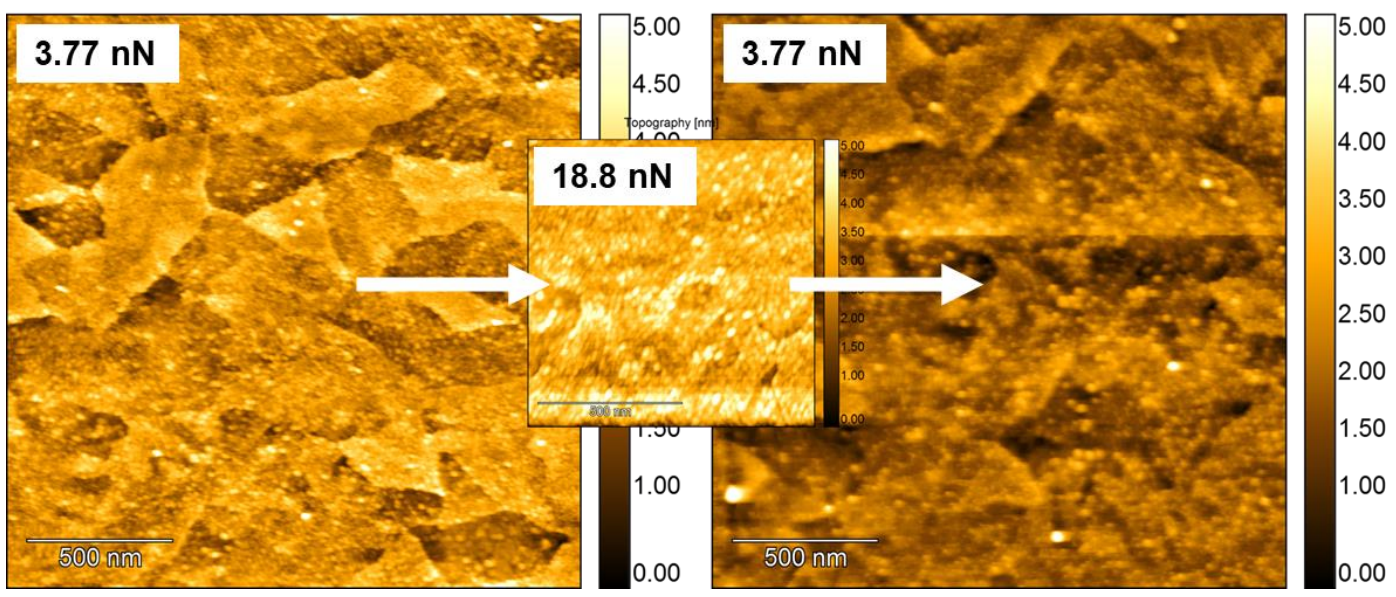

Figure S 33 AFM imaging in contact-mode confirms the presence of a self-assembled monolayer of 6-carboxylate on ITO by carving a small square using a high force. The ITO surface is imaged with a self-assembled monolayer of 6-carboxylate molecules (top) and without any molecules (bottom). The left two panels show scans of the ITO surfaces before carving using a relatively low force setpoint. The middle two smaller panels show areas zoomed-in onto the left panel at a high force setpoint. The right two panels are the same areas as on the left, after the nano-indentation. Only the surface with self-assembled molecules shows a carved patch in the monolayer after the experiment. 


\section{Current-time spectroscopy details}

For experiments with indium tin oxide (ITO) electrodes, glass substrates were purchased from SPI Supplies (USA) with a $700 \mathrm{~nm}$ layer of ITO. The exception is the AFM nanoindentation experiment, for which glass substrates with a $40 \mathrm{~nm}$ layer of ITO were used (Präzisions Glas \& Optik GmbH, PGO, Germany). The substrates from SPI Supplies were cut into squares of roughly $12 \times 12 \mathrm{~mm}$ with a diamond pen. They were cleaned according to Chockalingam et al. ${ }^{15}$ where they were first sonicated in DCM for $\sim 20$ minutes, then in methanol for another $\sim 20$ minutes, and finally for $\sim 30 \mathrm{~min}$ in a $0.5 \mathrm{M}$ solution of potassium carbonate in a 3:1 mixture of methanol:Milli- $Q$ water. The substrates were then immersed for $\sim 48$ hours in $1 \mathrm{mM}$ solutions of target molecules in 1:1 DCM:ethanol. STM-I(t) experiments for 6-carboxylate and 8-squarate were carried out in air, whereas TMB was added to the measurement of 7-acrylate.

A gold STM tip was freshly cut for each experiment and positioned a few angstroms from the substrate using a setpoint current of $\sim 5 \mathrm{nA}$. The feedback loop was then switched off to allow for fluctuations and jumps in the current to be monitored. Once the current was relatively stable, i.e. the baseline remains mostly horizontal, segments of 0.5 seconds were saved as raw data. Approximately 400 traces were saved for 6-carboxylate when stochastic current jumps were observed. Approximately 2000 traces for 7-acrylate and 8-squarate were saved regardless of whether any jumps were observed.

Raw I(t) traces were processed using two bespoke Python scripts (v.2.7, Python Software Foundation, https://www.python.org). The first script uses an automated routine to fit the background current to a sloped straight line that is then subtracted from the trace. After this process, the baseline current is zero, and the magnitude of the current jump can be established relative to the background. The processed traces were then used to compile the histograms shown in the main text, Figure 4A-C.

The processed traces were then subjected to a second algorithm, which aim is to "slice" the traces between abrupt changes in current (“jumps") to obtain additional information on the temporal stability of the junction. The algorithm calculates the second derivative of the smoothed (7-point moving median filter) current trace, and cuts (dumps data to a new file) when its value is above a pre-defined threshold. The algorithm also automatically rejects traces shorter than $1 \mathrm{~ms}$ as attributed to noise unfiltered by the moving median smoothing. These "slices" of data were then used to compile the density maps presented in the main text, Figure 4D-F. Details on the two algorithms, with example data and relative plots, can be found in the supporting information of our previous publications on the subject. ${ }^{16-17}$ 


\section{Transport calculations}

Figure S 34 shows two different possible binding configurations of $\mathrm{COO}^{-}$or $\mathrm{COOH}$ to an ITO.

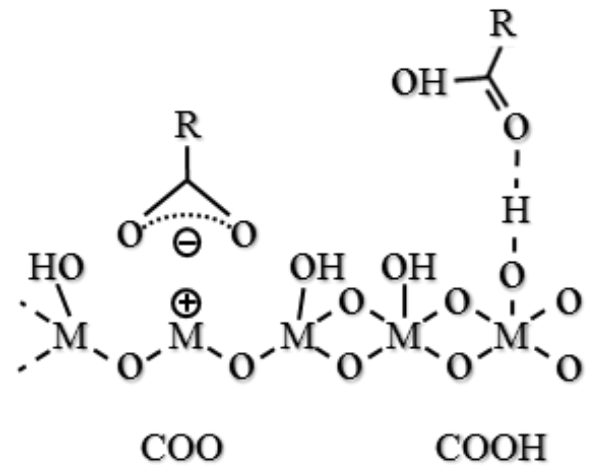

Configuration 1 Configuration2

Figure S 34 Possible binding structure of $\mathrm{COO}^{-}$or $\mathrm{COOH} \mathrm{H-bonding} \mathrm{groups} \mathrm{to} \mathrm{metal}$ oxide surfaces. Adapted with permission from reference ${ }^{18}$. Copyright 2014 John Wiley and Sons.

To find the most stable configuration, we have calculated the binding energy $E_{B}$ of the structures shown in Figure S 34 for all three molecules as illustrated in Table S1. We found that $\mathrm{COO}^{-}$has a stronger binding to ITO.

Table S1. Binding energy of COO- or $\mathrm{COH}$ groups to metal oxide surfaces.

\begin{tabular}{|l|l|l|}
\hline Possible binding configuration & Configuration1 & Configuration2 \\
\hline 6-carboxylate & $-3.2 \mathrm{eV}$ & $-0.54 \mathrm{eV}$ \\
\hline 7-acrylate & $-3.0 \mathrm{eV}$ & $-0.57 \mathrm{eV}$ \\
\hline 8-squarate & $-3.15 \mathrm{eV}$ & $-1.28 \mathrm{eV}$ \\
\hline
\end{tabular}

Figure $S 35$ shows the relaxed structure of the junctions for 6-carboxylate, 7-acrylate, and 8-squarate between gold and ITO electrodes using the COO- binding structure. To study the effect of the binding configuration on electrical conductance, we have then compared the electrical conductance of 6-carboxylate using the binding configuration 1 and 2 shown in Figure S 34. As shown in Figure S 36, the overall conductance of the configuration 2 is higher than that of 1 . Based on the binding energy calculations, the junction formation probability of $\mathrm{COOH}$ is expected to be lower than that of $\mathrm{COO}^{-}$, although both configurations are energetically possible at room temperature $\left(E_{B}>25 \mathrm{meV}\right)$. Therefore, we calculate the 
transmission coefficient for three different energetically optimised binding configurations of each molecule as shown in figure $S 37$. We found that the overall trend for the average conductances over different configurations are in agreement with experiment for a wide range of Fermi energies around DFT Fermi energy. As shown in table S2, the best agreement between experiment and theory is found at $E_{F}=-0.78 \mathrm{eV}$. We note that the calculations using ITO is computationally very demanding.

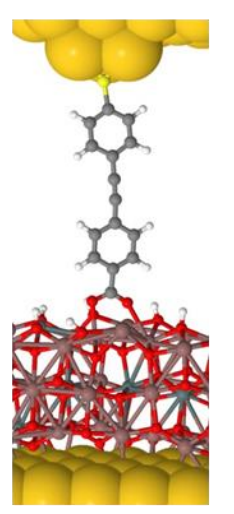

6-carboxylate

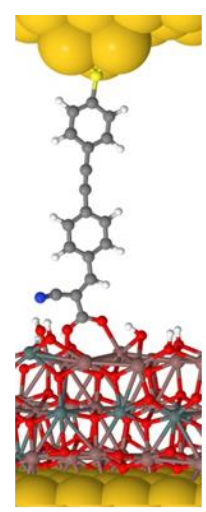

7-acrylate

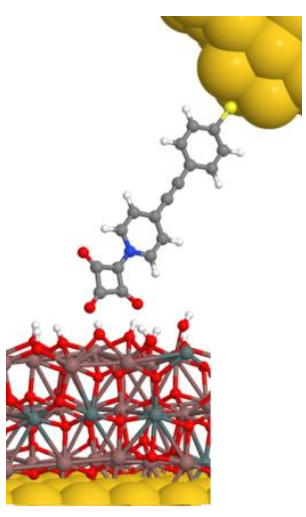

8-squarate

Figure S 35 Relaxed structure of the molecules connected to the gold and ITO. 
Table S2. Comparision between conductance in the units of $G_{0}$ between experimentally measured values and those calculated by averaging conductances for different configurations of each molecule (Figure S 37D). Although the trend predicted from DFT is in a good agreement with experiment, the calculated conductance values are higher than measured values. This is expected because DFT is well-known to overestimate the conductance values.

\begin{tabular}{|l|c|c|}
\hline \multirow{2}{*}{ Molecule } & \multicolumn{2}{|c|}{ Conductance $\left(\mathrm{G}_{0}\right)$} \\
\cline { 2 - 3 } & Calculation & Experiment \\
\hline 6-carboxylate & $1.1 \times 10^{-3}$ & $9.2 \times 10^{-5}$ \\
\hline 7-acrylate & $2.3 \times 10^{-4}$ & $5.9 \times 10^{-5}$ \\
\hline 8-squarate & $4.1 \times 10^{-4}$ & $7.2 \times 10^{-5}$ \\
\hline
\end{tabular}
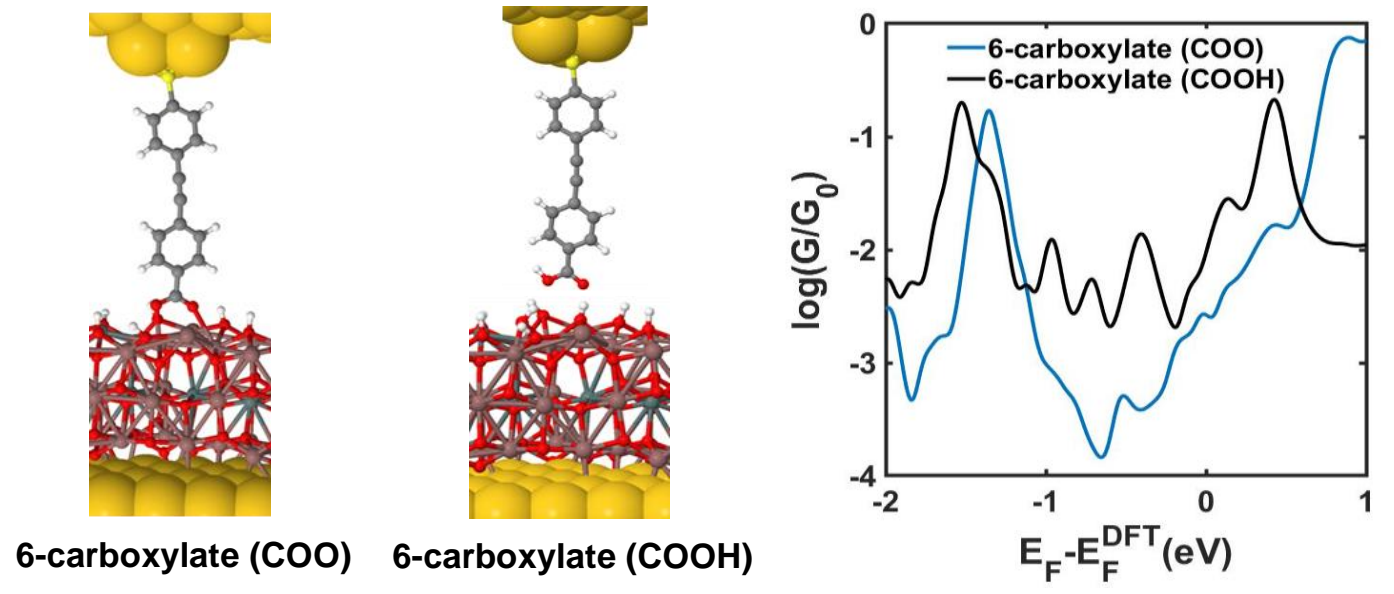

Figure S 36 Relaxed structure of the molecules connected to ITO using two different binding configurations and corresponding electrical conductances. 

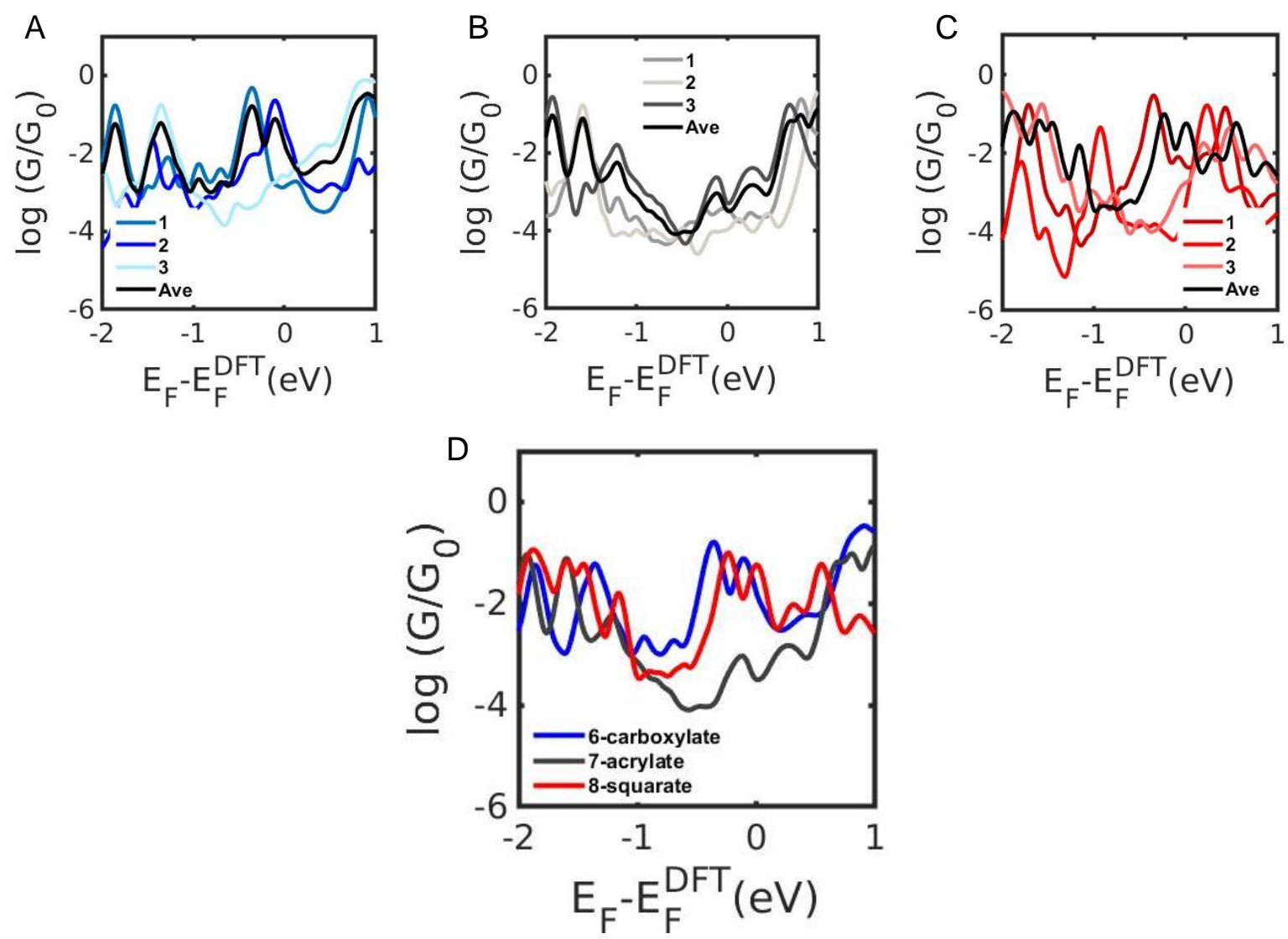

Figure S 37 A-C) Electrical conductances of the molecules 6-carboxylate, 7-acrylate and 8-squarate connected to ITO using three different configurations. D) Average of electrical conductances for the molecules.

We have also calculated the electrical conductance of 6-carboxylate, 7-acrylate, and 8squarate between gold-gold electrodes (Figure SA) and we found that the electrical conductance of 6-carboxylate is slightly higher than that of 7-acrylate (Figure SB) in agreement with the measurement (Figure $S$ 29). This is due to the HOMO level in 6carboxylate being closer to the Fermi energy compared to 7-acrylate, which leads to higher conductance. We also found that the electrical conductance of 8-squarate is lower than molecules 6 and 7. 
A

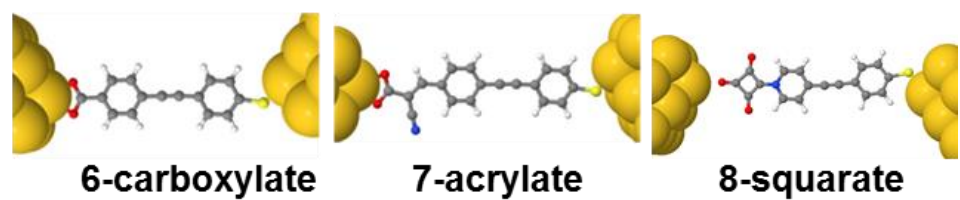

B

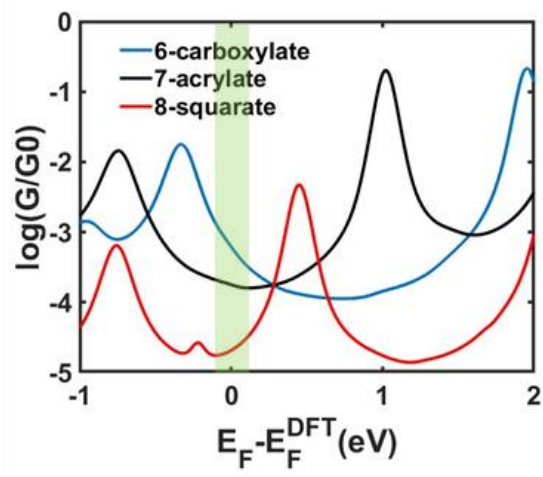

Figure S 38 A) Relaxed structure of the molecules between gold-gold electrodes. B) Electrical conductance of the molecules

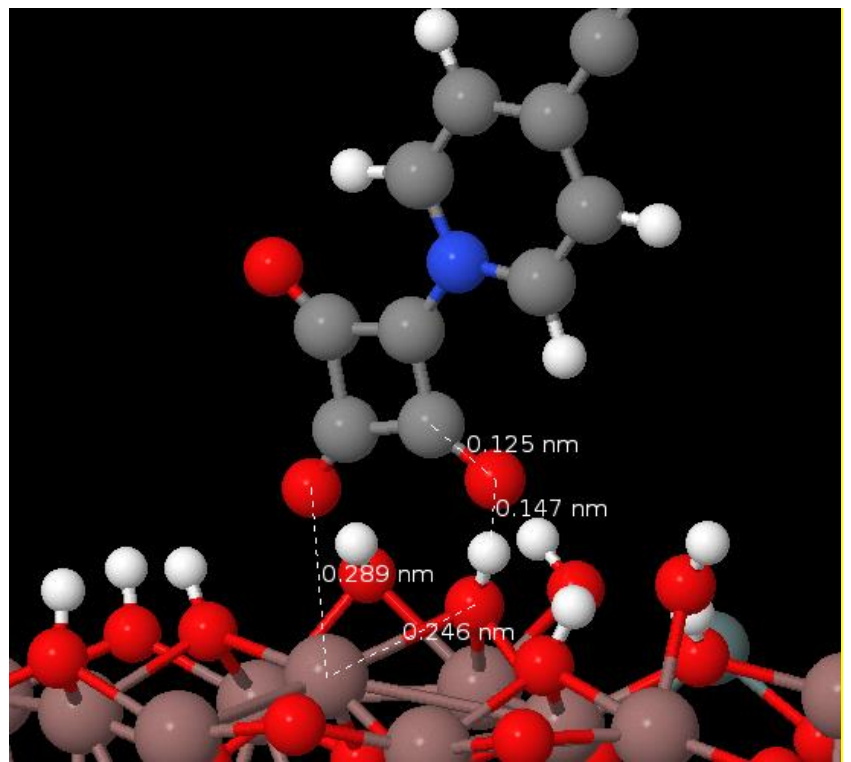

Figure S 39. An example of 8-squarate binding configuration to ITO. Two oxygen atoms interact with ITO. The bond length between one oxygen atom in squarate and the $\mathrm{H}$ atom of ITO indicates that a hydrogen bond has formed. The other oxygen atom in squarate interacts electrostatically with the surface of ITO. 


\section{Theoretical methods}

The optimised geometry, ground state Hamiltonian and overlap matrix element of each structure was self-consistently obtained using the SIESTA ${ }^{19}$ implementation of density functional theory (DFT). SIESTA employs norm-conserving pseudopotentials to account for the core electrons, and linear combinations of atomic orbitals to construct the valence states. The generalised gradient approximation (GGA) of the exchange and correlation functional is used with the Perdew-Burke-Ernzerhof parameterisation (PBE) a double- $\zeta$ polarised (DZP) basis set, a real-space grid defined with an equivalent energy cut-off of 250 Ry. The geometry optimisation for each structure is performed to achieve forces smaller than $10 \mathrm{meV}$ / $\AA$.

The mean-field Hamiltonian obtained from the converged DFT calculation was combined with Gollum ${ }^{20}$ implementation of the non-equilibrium Green's function method ${ }^{21}$ to calculate the phase-coherent, elastic scattering properties of each system consisting of left gold (source) and right gold (drain) leads and the scattering region. The transmission coefficient $T(E)$ for electrons of energy $E$ (passing from the source to the drain) is calculated via the relation: $T(E)=\operatorname{Trace}\left(\Gamma_{R}(E) G^{R}(E) \Gamma_{L}(E) G^{R \dagger}(E)\right)$. In this expression, $\Gamma_{L, R}(E)=i\left(\sum_{L, R}(E)-\right.$ $\left.\sum_{L, R}{ }^{\dagger}(E)\right)$ describe the level broadening due to the coupling between left $(L)$ and right $(R)$ electrodes and the central scattering region, $\sum_{L, R}(E)$ are the retarded self-energies associated with this coupling and $G^{R}=\left(E S-H-\sum_{L}-\sum_{R}\right)^{-1}$ is the retarded Green's function, where $H$ is the Hamiltonian and $S$ is overlap matrix. Using obtained transmission coefficient $T(E)$, the conductance could be calculated by Landauer formula $\left(G=G_{0} \int d E T(E)(-\partial f / \partial E)\right)$ where $G_{0}=2 e^{2} / h$ is conductance quantum. 


\section{References}

1. Oh, S. Y.; Chung, C. M.; Kim, D. H.; Lee, S. G., Characteristics and fabrication of oligophenyleneethynylene thiol self-assembled monolayers. Colloids and Surfaces A: Physicochemical and Engineering Aspects 2008, 313-314, 600-603.

2. Meisner, J. S.; Sedbrook, D. F.; Krikorian, M.; Chen, J.; Sattler, A.; Carnes, M. E.; Murray, C. B.; Steigerwald, M.; Nuckolls, C., Functionalising molecular wires: a tunable class of $\alpha, \omega$-diphenyl- $\mu, v$-dicyano-oligoenes. Chemical Science 2012, 3 (4), 1007-1014.

3. Arakawa, Y.; Kang, S.; Tsuji, H.; Watanabe, J.; Konishi, G.-i., The design of liquid crystalline bistolane-based materials with extremely high birefringence. RSC Advances 2016, 6 (95), 92845-92851.

4. Olivier, J.-H.; Haefele, A.; Retailleau, P.; Ziessel, R., Borondipyrromethene Dyes with Pentane-2,4-dione Anchors. Organic Letters 2010, 12 (3), 408-411.

5. Inagaki, S.; Saito, K.; Suto, S.; Aihara, H.; Sugawara, A.; Tamura, S.; Kawano, T., Synthesis of 5-Aryl-3(2H)-furanones Using Intramolecular Cyclisation of Sulfonium Salts. The Journal of Organic Chemistry 2018, 83 (22), 13834-13846.

6. Bartucci, M. A.; Wierzbicki, P. M.; Gwengo, C.; Shajan, S.; Hussain, S. H.; Ciszek, J. W., Synthesis of dihydroindolizines for potential photoinduced work function alteration. Tetrahedron Letters 2010, 51 (52), 6839-6842.

7. Mészáros, G.; Li, C.; Pobelov, I.; Wandlowski, T., Current measurements in a wide dynamic range-applications in electrochemical nanotechnology. Nanotechnology 2007, 18 (42), 424004.

8. Huang, W. Y.; Chen, H. Y., Synthesis and Characterization of a Low-Bandgap Poly(arylene ethynylene) Having Donor-Acceptor Type Chromophores in the Side Chain. Macromolecules 2013, 46 (5), 2032-2037.

9. Tseng, H. R.; Vignon, S. A.; Stoddart, J. F., Toward chemically controlled nanoscale molecular machinery. Angewandte Chemie-International Edition 2003, 42 (13), 1491-1495.

10. Sander, F.; Hermes, J. P.; Mayor, M.; Hamoudi, H.; Zharnikov, M., Add a third hook: S-acetyl protected oligophenylene pyridine dithiols as advanced precursors for selfassembled monolayers. Physical Chemistry Chemical Physics 2013, 15 (8), 2836-2846.

11. Heister, K.; Zharnikov, M.; Grunze, M.; Johansson, L. S. O., Adsorption of alkanethiols and biphenylthiols on $\mathrm{Au}$ and $\mathrm{Ag}$ substrates: $\mathrm{A}$ high-resolution X-ray photoelectron spectroscopy study. Journal of Physical Chemistry B 2001, 105 (19), 40584061.

12. Escorihuela, E.; Cea, P.; Bock, S.; Milan, D. C.; Naghibi, S.; Osorio, H. M.; Nichols, R. J.; Low, P. J.; Martin, S., Towards the design of effective multipodal contacts for use in 
the construction of Langmuir-Blodgett films and molecular junctions. J. Mater. Chem. C 2020, 8 (2), 672-682.

13. Beamson, G.; Briggs, D., High Resolution XPS of organic polymers: the Scienta ESCA300 Database, p.222 John Wiley, Chichester, England 1992.

14. Alvaro, M.; García, H.; García, S.; Márquez, F.; Scaiano, J. C., Intrazeolite Photochemistry. 17. Zeolites as Electron Donors: Photolysis of Methylviologen Incorporated within Zeolites. The Journal of Physical Chemistry B 1997, 101 (16), 3043-3051.

15. Chockalingam, M.; Darwish, N.; Le Saux, G.; Gooding, J. J., Importance of the Indium Tin Oxide Substrate on the Quality of Self-Assembled Monolayers Formed from Organophosphonic Acids. Langmuir 2011, 27 (6), 2545-2552.

16. Vezzoli, A.; Brooke, R. J.; Ferri, N.; Higgins, S. J.; Schwarzacher, W.; Nichols, R. J., Single-Molecule Transport at a Rectifying GaAs Contact. Nano Lett. 2017, 17 (2), 11091115.

17. Vezzoli, A.; Brooke, R. J.; Higgins, S. J.; Schwarzacher, W.; Nichols, R. J., SingleMolecule Photocurrent at a Metal-Molecule-Semiconductor Junction. Nano Lett. 2017, 17 (11), 6702-6707.

18. Pujari, S. P.; Scheres, L.; Marcelis, A. T. M.; Zuilhof, H., Covalent Surface Modification of Oxide Surfaces. Angewandte Chemie International Edition 2014, 53 (25), 6322-6356.

19. Soler, J. M.; Artacho, E.; Gale, J. D.; García, A.; Junquera, J.; Ordejón, P.; SánchezPortal, D., The SIESTA method forab initioorder-Nmaterials simulation. Journal of Physics: Condensed Matter 2002, 14 (11), 2745-2779.

20. Ferrer, J.; Lambert, C. J.; García-Suárez, V. M.; Manrique, D. Z.; Visontai, D.; Oroszlany, L.; Rodríguez-Ferradás, R.; Grace, I.; Bailey, S. W. D.; Gillemot, K.; Sadeghi, H.; Algharagholy, L. A., GOLLUM: a next-generation simulation tool for electron, thermal and spin transport. New Journal of Physics 2014, 16 (9), 093029.

21. Sadeghi, H., Theory of electron, phonon and spin transport in nanoscale quantum devices. Nanotechnology 2018, 29 (37), 373001. 